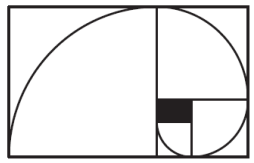

\title{
La notion de stabilité pour des équations aux dérivées partielles elliptiques
}

\author{
Olivier Druet
}

Résumé. Ce texte est issu d'un mémoire d'habilitation à diriger des recherches. Nous y introduisons la notion de stabilité d'une équation aux dérivées partielles elliptiques, notion différente de celle plus classique de stabilité d'une solution d'une équation. Nous étudierons précisément cette notion sur un exemple et donnerons rapidement quelques résultats à propos d'autres équations.

2010 Mathematics Subject Classification : 35B44, 35B45, 35J30, 35J47, 35J60, 35Q75, 58J05. 



\section{Table des matières}

1 Introduction

1.1 Qu'est-ce que la stabilité d'une EDP

elliptique . . . . . . . . . . . . . . . 6

1.2 Distance pointée entre sous-ensembles

de $C^{2} \ldots \ldots \ldots \ldots \ldots \ldots \ldots \ldots$

1.3 L'équation de Poisson . . . . . . . . . . . . . . . . . . . . . . . . . . . . . 8

1.4 Une équation linéaire . . . . . . . . . . . . . . . . . . . . . . 8

1.5 Une équation non-linéaire sous-critique . . . . . . . . . . . . 9

2 Etude détaillée d'un exemple : les équations de type Yamabe 15

2.1 Le problème de Yamabe . . . . . . . . . . . . . . . . . 15

2.2 Compacité . . . . . . . . . . . . . . . . . . 18

2.3 Stabilité - Définitions, exemples . . . . . . . . . . . . 19

2.4 Résultats de stabilité et d'instabilité . . . . . . . . . . . . . 23

2.5 Lieux d'instabilité . . . . . . . . . . . . . . . . . . . 26

2.6 Et si les solutions changent de signe? . . . . . . . . . . . . . 28

3 Analyse asymptotique - un outil pour la stabilité 29

3.1 Description ponctuelle des défauts de

compacité . . . . . . . . . . . . . . . 29

3.1.1 Comment trouver les points de concentration? . . . 35

3.1.2 Un premier contrôle ponctuel de la suite . . . . . . 45

3.1.3 Hiérarchie des points de concentration et contrôle

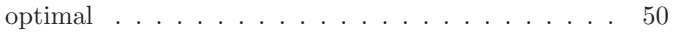

3.1.4 Asymptotique précise . . . . . . . . . . . . . . 51

3.1.5 Conclusion .................. . . 52

3.2 Et si l'opérateur a un noyau? . . . . . . . . . . . . . . . . . 52

3.3 Rayons d'influence des bulles . . . . . . . . . . . . . . . . 52

3.4 Preuve du théorème $2.1 \ldots$. . . . . . . . . . . . . . . . 55

3.5 Et si l'énergie n'est pas bornée? . . . . . . . . . . . 56 
4 D'autres équations $\quad 58$

4.1 L'équation d'Einstein-Lichnerowicz . . . . . . . . . . . . . . 58

4.1.1 Les contraintes dans la théorie d'Einstein avec champ

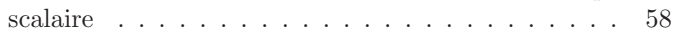

4.1 .2 Stabilité et instabilité . . . . . . . . . . . . . 64

4.2 Systèmes d'EDP elliptiques . . . . . . . . . . . . . . . . 65

4.3 Stabilité de l'obstruction de Pohožaev . . . . . . . . . . . . 69

4.4 Des équations d'ordre $4 \ldots \ldots$. . . . . . . . . . . . . . . . . . . . . . . 77

4.5 Une non-linéarité en $e^{u^{2}} \ldots$. . . . . . . . . . . . . . . . 82

4.5.1 Le premier point de concentration . . . . . . . 85

4.5.2 Comment trouver d'autres points de concentration? 87

4.5.3 Toute l'énergie a-t-elle été trouvée? . . . . . . . . . 90

4.5 .4 Et après ? . . . . . . . . . . . . . . . . . . . 91

4.5.5 Avec le bilaplacien ... . . . . . . . . . . . . . . . . 92 


\section{Chapitre 1}

\section{Introduction}

Ce texte est consacré à la notion de stabilité pour des équations aux dérivées partielles elliptiques. Cette notion a vu le jour dans une série de papiers $[40,41,52]$, même si elle était déjà latente auparavant. Elle permet de jeter une nouvelle lumière sur un certain nombre de travaux antérieurs et nous paraît extrêmement intéressante à étudier pour elle-même.

En deux mots, la question posée à propos d'une équation est la suivante : si on perturbe l'équation, est-ce que l'ensemble des solutions change beaucoup ou non? Si les solutions de l'équation perturbées sont toutes proches d'une solution de l'équation de départ, nous dirons que l'équation est stable. Si certaines perturbations créent des solutions nouvelles, loin de toute solution de l'équation de départ, nous dirons que celle-ci est instable.

Il nous faut bien entendu préciser ce que nous entendons par "perturbations", par "proche de", ... Ceci sera fait au coup par coup au cours de ce texte. Nous avons pris le parti de ne pas donner de définition générale de la stabilité, pour des équations quelconques, afin de laisser à cette notion un caractère multiforme et peu figé. Bien entendu, pour chaque équation considérée, nous donnerons des définitions précises et nous espérons que les quelques exemples traités dans ce texte finiront par donner une idée assez claire de cette notion.

Ce texte est organisé comme suit : dans le premier chapitre, nous discutons de cette notion de stabilité d'une EDP elliptique en prenant quelques exemples simples, voire triviaux. Nous la distinguons en particulier de la notion plus classique de stabilité d'une solution d'une EDP elliptique. Le deuxième chapitre est consacré à l'étude de cette notion sur une équation modèle. Nous prenons cet exemple pour des raisons à la fois historique son étude a été un des moteurs de l'analyse géométrique ces quarante dernières années et a été à l'origine de la majeure partie des outils utilisés pour étudier la stabilité - et pratique - c'est l'équation sur laquelle le plus de résultats sont disponibles. Ce deuxième chapitre donnera une 
idée précise des questions qui se posent autour de cette notion de stabilité et des résultats obtenus jusqu'ici. Dans celui-ci, nous ne donnerons aucune preuve, ni même aucune esquisse de preuve. Le troisième chapitre sera consacré à la description des outils nécessaires à l'étude de la stabilité d'une EDP elliptique. Nous y esquisserons quelques preuves de résultats énoncés dans le second chapitre ${ }^{1}$. Enfin, dans le dernier chapitre, afin de mieux cerner cette nouvelle notion, nous passerons en revue quelques autres équations dont la stabilité a été étudiée.

\subsection{Qu'est-ce que la stabilité d'une EDP elliptique?}

Dans ce chapitre, nous tentons de cerner la notion de stabilité d'une équation aux dérivées partielles elliptique en partant d'équations simples. Avant même de parler d'EDP elliptiques, nous allons faire une analogie (osée et risquée) avec les points critiques d'une variable réelle à valeurs réelles. Intéressons-nous aux points critiques d'une fonction régulière de $\mathbb{R}$ dans $\mathbb{R}$. Etant donnée $f \in C^{1}(\mathbb{R}, \mathbb{R})$, nous noterons $C r i t(f)$ l'ensemble de ses points critiques, i.e. l'ensemble des $x \in \mathbb{R}$ tels que $f^{\prime}(x)=0$. On peut se demander si perturber dans $C^{1}$ la fonction $f$ peut créer des nouveaux points critiques loin de tout point critique de $f$. Si c'est le cas, nous dirions que l'équation $f^{\prime}(x)=0$ est instable; nous dirions qu'elle est stable dans le cas contraire. Illustrons ce propos par deux exemples :

Tout d'abord considérons la fonction $f(x)=x^{3}$. Elle possède un seul point critique, en $x=0$, donc $\operatorname{Crit}(f)=\{0\}$. Ce point critique est instable en tant que point critique puisque la fonction $f_{\varepsilon}(x)=x^{3}+\varepsilon x$ pour $\varepsilon>0$ ne possède elle aucun point critique. Par contre, l'équation $f^{\prime}(x)=0$ est elle stable dans le sens que nous donnerons à la stabilité dans ce texte. En effet, si $f_{\varepsilon} \rightarrow f$ dans $C^{1}(\mathbb{R})$ quand $\varepsilon \rightarrow 0$ et si $x_{\varepsilon} \in C$ rit $\left(f_{\varepsilon}\right)$, alors $x_{\varepsilon} \rightarrow 0$ quand $\varepsilon \rightarrow 0$. Nulle perturbation de $f$ dans $C^{1}$ ne crée de points critiques loin du seul point critique de la fonction initiale.

Comme deuxième exemple, nous prendrons la fonction $f(x)=\frac{x}{1+x^{2}}$. Alors $\operatorname{Crit}(f)=\{-1,+1\}$. Ces deux points critiques, en tant que point critique, sont stables. Par contre, l'équation $f^{\prime}(x)=0$ est elle instable. En effet, la suite de fonctions définies pour $\varepsilon>0$ par $f_{\varepsilon}=f+\varepsilon \sin ($. converge vers $f$ in $C^{1}(\mathbb{R})$ quand $\varepsilon \rightarrow 0$ et ces fonctions $f_{\varepsilon}$ possèdent des points critiques sur tout intervalle de la forme $[M,+\infty)$. Donc on peut perturber $f$ dans $C^{1}$ de façon à créer de nouveaux points critiques loin des points critiques de $f$.

1. Le lecteur peut choisir de lire le chapitre 3 avant le chapitre 2. Celui-ci, qui traite d'analyse asymptotique de suites de solutions d'EDP elliptiques, décrit des résultats intéressants indépendamment de leur application à la stabilité. 
Ces deux exemples n'avaient pas pour but d'illustrer ce qui se passe pour des solutions d'équations aux dérivées partielles. Ils sont bien entendu beaucoup trop simplistes pour cela. Par contre, ils permettent de pointer la différence entre stabilité d'une solution (d'un point critique) et stabilité de l'équation (de l'ensemble des points critiques).

Considérons l'équation très générale du type

$$
\Delta u=f(x, u)
$$

sur un domaine de $\mathbb{R}^{n}$ (avec conditions au bord) ou sur une variété. Nous dirons que cette équation est stable si une petite perturbation de $f$ ne crée pas de solutions nouvelles loin des solutions de l'équation de départ. En d'autres termes, l'équation sera dite stable si

$$
f_{\varepsilon} \rightarrow f \text { quand } \varepsilon \rightarrow 0 \Longrightarrow \mathcal{S}_{\varepsilon} \rightarrow \mathcal{S} \text { quand } \varepsilon \rightarrow 0
$$

où $\mathcal{S}_{\varepsilon}$ et $\mathcal{S}$ désignent respectivement les ensembles de solutions des équations perturbées et de l'équation initiale. Il nous faudra préciser quelle classe de perturbations est autorisée ${ }^{2}$ et préciser ce que nous entendons par convergence d'ensemble. Comme nous l'avons déjà dit, la notion de stabilité d'une équation n'a strictement rien à voir avec celle de stabilité d'une solution. Une solution de l'équation ci-dessus sera dite stable $^{3} \mathrm{si}$ l'opérateur linéarisé en $u$ n'a que des valeurs propres positives. L'opérateur linéarisé est ici donné par $v \mapsto \Delta v-\frac{\partial f}{\partial u}(x, u) v$. De même que pour les points critiques de fonctions d'une variable réelle, une équation peut être instable alors qu'elle n'a que des solutions stables (et réciproquement).

\subsection{Distance pointée entre sous-ensembles de $C^{2}$}

Pour être plus précis, il va nous falloir introduire une distance entre les ensembles de solutions des équations. Pour $X$ et $Y$ deux sous-ensembles de $C^{2}(M)$, nous définirons donc la distance pointée suivante :

$$
d_{C^{2}}^{\hookrightarrow}(X ; Y)=\sup _{u \in X} \inf _{v \in Y}\|v-u\|_{C^{2}(M)}
$$

Ce n'est pas une vraie distance puisqu'elle n'est pas symétrique ${ }^{4}$. Par convention, nous poserons $d_{C^{2}}^{\hookrightarrow}(X, \emptyset)=+\infty$ si $X \neq \emptyset$ et $d_{C^{2}}^{\hookrightarrow}(\emptyset, Y)=0$ pour tout $Y$.

\footnotetext{
2. Chaque type de perturbations mène à sa propre question de stabilité.

3. Même pour une solution, il existe beaucoup de notions de stabilité ...

4. Cette distance est nulle si et seulement si $X \subset \bar{Y}$. Elle vérifie une inégalité triangulaire $d_{C^{2}}^{\hookrightarrow}(X ; Z) \leq d_{C^{2}}^{\hookrightarrow}(X ; Y)+d_{C^{2}}^{\hookrightarrow}(Y ; Z)$.
} 


\subsection{L'équation de Poisson}

Considérons $\Omega$ un domaine (ouvert borné régulier) de $\mathbb{R}^{n}, n \geq 2$. Pour $f \in C^{\infty}\left(\mathbb{R}^{n}\right)$, nous nous intéressons dans cette section à la stabilité de l'équation

$$
\Delta u=f \text { dans } \Omega, u=0 \text { sur } \partial \Omega .
$$

Etant donnée la fonction $f$, il existe une unique solution $u(f)$ à l'équation (1.2). De plus, cette solution est régulière et vérifie par exemple

$$
\|u(f)\|_{C^{2}(\bar{\Omega})} \leq C\|f\|_{C^{1}(\bar{\Omega})}
$$

pour une certaine constante $C>0$ indépendante de $f$. Cette estimée n'est pas optimale (en termes de régularité de $f$ ) mais cela n'a pas grande importance pour notre propos. Ainsi, étant données deux fonctions $f_{1}$ et $f_{2}$, les deux solutions $u\left(f_{1}\right)$ et $u\left(f_{2}\right)$ de $(1.2)$ associées vérifient

$$
\left\|u\left(f_{1}\right)-u\left(f_{2}\right)\right\|_{C^{2}(\bar{\Omega})} \leq C\left\|f_{1}-f_{2}\right\|_{C^{1}(\bar{\Omega})} .
$$

Il est ainsi clair que l'ensemble des solutions de (1.2) ne varie pas trop par perturbation $C^{1}$ de $f$. Plus précisément, pour tout $\varepsilon>0$, il existe $\delta>0$ tel que,

$$
\|f-\tilde{f}\|_{C^{1}(\bar{\Omega})} \leq \delta \Longrightarrow\|u(\tilde{f})-u(f)\|_{C^{2}(\bar{\Omega})} \leq \varepsilon,
$$

ce qui entraîne

$$
\|f-\tilde{f}\|_{C^{1}(\bar{\Omega})} \leq \delta \Longrightarrow d_{C^{2}}^{\hookrightarrow}\left(\mathcal{S}_{\tilde{f}} ; \mathcal{S}_{f}\right) \leq \varepsilon
$$

où $\mathcal{S}_{f}=\{u(f)\}$ est l'ensemble des solutions de (1.2). Nous dirons donc que l'équation (1.2) est stable (par perturbations $C^{1}$ de $f$ ).

Bien entendu, cet exemple est trivial puisque l'ensemble des solutions de (1.2) est réduit à un élément. Nous allons complexifier un peu cet exemple dans les sections suivantes.

\subsection{Une équation linéaire}

Considérons $\Omega$ un domaine (ouvert borné régulier) de $\mathbb{R}^{n}, n \geq 2$. Pour $f \in C^{\infty}\left(\mathbb{R}^{n}\right)$, nous nous intéressons dans cette section à la stabilité de l'équation

$$
\Delta u=f u \text { dans } \Omega, u=0 \text { sur } \partial \Omega
$$


Nous noterons $\mathcal{S}_{f}$ l'ensemble des solutions de l'équation (1.3). Cet ensemble est un espace vectoriel de dimension finie s'il n'est pas vide, ce qui peut arriver. En particulier,

$$
d_{C^{2}}^{\hookrightarrow}\left(\mathcal{S}_{\tilde{f}} ; \mathcal{S}_{f}\right)=+\infty
$$

dès que $\mathcal{S}_{\tilde{f}} \neq \emptyset$ et $f \neq \tilde{f}$. En effet, c'est vrai par convention si $\mathcal{S}_{f}=\emptyset$; sinon, en utilisant la structure d'espace vectoriel de $\mathcal{S}_{f}$ et $\mathcal{S}_{\tilde{f}}$, on peut écrire que

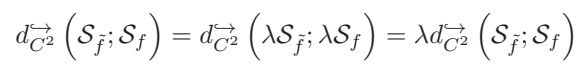

pour tout $\lambda>0$, ce qui n'est possible que si cette distance est nulle ou infinie. Or elle ne peut être nulle que si $f=\tilde{f}$.

Ainsi, il est clair que l'équation (1.3) ne peut pas être stable par perturbations de $f$ si $\mathcal{S}_{f} \neq \emptyset$. En effet, dès que l'on perturbe $f$ par une fonction $\tilde{f} \neq f$ telle que $\mathcal{S}_{\tilde{f}} \neq \emptyset$, ce qui est toujours possible dans $C^{1}(\Omega)$ si $\mathcal{S}_{f} \neq \emptyset$, la distance de l'ensemble $\mathcal{S}_{\tilde{f}}$ à l'ensemble $\mathcal{S}_{f}$ est infinie.

Comme il serait tout de même agréable de pouvoir dire qu'une équation linéaire est stable, il va nous falloir affiner légèrement notre notion de stabilité. Un bon moyen de retrouver de la stabilité pour cette équation est de se limiter aux solutions d'énergie bornée. Posons

$$
\mathcal{S}_{f}^{\Lambda}=\left\{u \text { solution de (1.3) avec } \int_{\Omega}|\nabla u|^{2} d x \leq \Lambda\right\} .
$$

Il est alors simple de montrer ${ }^{5}$ que l'équation est stable dans le sens suivant : pour tout $\Lambda>0$, pour tout $\varepsilon>0$, il existe $\delta>0$ tel que

$$
\|\tilde{f}-f\|_{C^{1}} \leq \delta \Longrightarrow d_{C^{2}}^{\hookrightarrow}\left(\mathcal{S}_{\tilde{f}}^{\Lambda} ; \mathcal{S}_{f}^{\Lambda}\right) \leq \varepsilon
$$

Ainsi l'instabilité de l'équation linéaire n'est due qu'aux solutions dont l'énergie est (infiniment) grande. Dès qu'une borne a priori sur l'énergie est donnée, l'équation redevient stable. Nous verrons qu'il est souvent, même pour des équations non-linéaires, important de distinguer ces deux types de stabilité, avec ou sans bornes sur l'énergie.

\subsection{Une équation non-linéaire sous-critique}

Passons maintenant à une équation un peu plus intéressante. Considérons $\Omega$ un domaine de $\mathbb{R}^{n}, n \geq 3$, et $h \in C^{\infty}\left(\mathbb{R}^{n}\right)$. Nous allons étudier la stabilité de l'équation

$$
\Delta u+h u=u^{q-1} \text { dans } \Omega, u>0 \text { dans } \Omega \text { et } u=0 \text { sur } \partial \Omega
$$

5. Le lecteur pourra consulter la première partie de la preuve de la proposition 1.1 qui s'applique ici aussi. 
par perturbations $C^{1}$ du potentiel $h$. Ici, $q$ sera un exposant sous-critique, c'est-à-dire $2<q<2^{\star}=\frac{2 n}{n-2}$. Cette équation est non-linéaire à croissance sous-critique. Nous rappelons que l'espace de Sobolev $H_{0}^{1}(\Omega)$, la complétion de l'ensemble des fonctions régulières à support compact dans $\Omega$ pour la norme $L^{2}$ du gradient, s'injecte continûment dans $L^{p}(\Omega)$ pour tout $p \leq 2^{\star}=\frac{2 n}{n-2}$ et que, de plus, l'injection est compacte pour $p<2^{\star}$. C'est cette compacité de l'injection de $H_{0}^{1}(\Omega)$ dans $L^{q}(\Omega)$ qui vaut son appellation sous-critique à l'équation (1.4) pour $2<q<2^{\star}$. Si on prend la convention $2^{\star}=+\infty$ pour $n=2$, tout ce qui sera dit ci-dessous pourrait être étendu au cas $n=2$ mais nous laisserons les preuves au lecteur.

Nous noterons $\mathcal{S}_{h}$ l'ensemble des solutions de l'équation (1.4). Cet ensemble est non-vide si et seulement si l'opérateur $\Delta+h$ (avec condition de Dirichlet au bord) est coercif. En effet, soit $\varphi$ une fonction propre associée à la première valeur propre $\lambda_{1}$ de $\Delta+h$ sur $\Omega$ avec condition de Dirichlet au bord. Cette fonction $\varphi$ peut-être choisie strictement positive dans $\Omega$. On a alors, en multipliant l'équation (1.4) par $\varphi$ et en intégrant par parties

$$
\begin{aligned}
\int_{\Omega} u^{q-1} \varphi d x & =\int_{\Omega}(\Delta u+h u) \varphi d x \\
& =\int_{\Omega}(\Delta \varphi+h \varphi) u d x \\
& =\lambda_{1} \int_{\Omega} u \varphi d x,
\end{aligned}
$$

ce qui entraîne $\lambda_{1}>0$ puisque $u>0$ et $\varphi>0$ dans $\Omega$. Donc l'équation (1.4) ne peut avoir une solution que si l'opérateur $\Delta+h$ est coercif. Réciproquement, si l'opérateur est coercif, alors le problème de minimisation

$$
\mu_{h}=\inf _{u \in H_{0}^{1}(\Omega), u \neq 0} \frac{\int_{\Omega}\left(|\nabla u|^{2}+h u^{2}\right) d x}{\left(\int_{\Omega}|u|^{q} d x\right)^{\frac{2}{q}}}
$$

admet une solution si l'exposant $2<q<2^{\star}$. Ce résultat s'obtient simplement en prenant une suite minimisante $\left(u_{i}\right)$, que l'on peut choisir positive ou nulle, normalisée par $\int_{\Omega} u_{i}^{q} d x=1$. Comme l'opérateur est coercif, on en déduit qu'elle est uniformément bornée dans $H_{0}^{1}(\Omega)$ et qu'on peut en extraire une sous-suite qui converge faiblement dans $H_{0}^{1}(\Omega)$ vers une fonction $u$ qui continue à vérifier $\int_{\Omega} u^{q} d x=1$ puisque l'inclusion de $H_{0}^{1}(\Omega)$ dans $L^{q}(\Omega)$ est compacte pour $q<2^{\star}$. Comme un passage à la limite faible fait au pire perdre de l'énergie, il est clair que $u$ est solution du problème de minimisation, non-nulle puisque $\int_{\Omega} u^{q} d x=1$. L'équation d'Euler-Lagrange de ce problème est l'équation (1.4) à un multiplicateur de Lagrange près. Il suffit ensuite de multiplier $u$ par la constante adéquate pour obtenir une solution de (1.4). Sa stricte positivité vient ensuite du principe du maximum. 
Nous allons démontrer le résultat suivant :

Proposition 1.1. L'équation (1.4) est stable par perturbations $C^{1}$ de $h$. En d'autres termes, pour tout $\varepsilon>0$, il existe $\delta>0$ tel que

$$
\|h-\tilde{h}\|_{C^{1}(\bar{\Omega})} \leq \delta \Longrightarrow d_{C^{2}}^{\hookrightarrow}\left(\mathcal{S}_{\tilde{h}} ; \mathcal{S}_{h}\right) \leq \varepsilon
$$

Preuve de la proposition 1.1 - Nous allons commencer par démontrer la stabilité à énergie bornée puis nous passerons au cas général ensuite. Nous noterons, pour $\Lambda>0$,

$$
\mathcal{S}_{h}^{\Lambda}=\left\{u \in C^{2}(\Omega) \text { solution de (1.4) avec } \int_{\Omega} u^{q} d x \leq \Lambda\right\} .
$$

Nous commencerons par montrer que pour tout $\varepsilon>0$, il existe $\delta>0$ tel que

$$
\|h-\tilde{h}\|_{C^{1}(\bar{\Omega})} \leq \delta \Longrightarrow d_{C^{2}}^{\hookrightarrow}\left(\mathcal{S}_{\tilde{h}}^{\Lambda} ; \mathcal{S}_{h}^{\Lambda}\right) \leq \varepsilon
$$

Le résultat annoncé correspond au cas $\Lambda=+\infty$ que nous démontrerons ensuite.

Pour démontrer la stabilité à énergie bornée, nous allons procéder par l'absurde. Fixons $\Lambda>0$. Supposons que l'équation n'est pas stable au niveau d'énergie $\Lambda$, i.e. qu'il existe une suite $\left(h_{\alpha}\right)_{\alpha \in \mathbb{N}}$ de fonctions régulières vérifiant

$$
h_{\alpha} \rightarrow h \text { dans } C^{1}(\bar{\Omega}) \text { quand } \alpha \rightarrow+\infty
$$

et $\varepsilon_{0}>0$ tels que

$$
d_{C^{2}}^{\hookrightarrow}\left(\mathcal{S}_{h_{\alpha}}^{\Lambda} ; \mathcal{S}_{h}^{\Lambda}\right) \geq \varepsilon_{0}
$$

pour tout $\alpha$. Ceci signifie en particulier qu'il existe une suite $\left(u_{\alpha}\right)$ de solutions de

$$
\Delta u_{\alpha}+h_{\alpha} u_{\alpha}=u_{\alpha}^{q-1} \text { dans } \Omega, u_{\alpha}>0 \text { dans } \Omega \text { et } u_{\alpha}=0 \text { sur } \partial \Omega
$$

avec

$$
\int_{\Omega} u_{\alpha}^{q} d x \leq \Lambda
$$

telle que

$$
d_{C^{2}}\left(u_{\alpha}, \mathcal{S}_{h}^{\Lambda}\right) \geq \frac{1}{2} \varepsilon_{0} .
$$

Multiplions l'équation (1.5) par $u_{\alpha}^{k-1}$ pour $k \geq 2$ et intégrons par parties pour obtenir

$$
(k-1) \int_{\Omega} u_{\alpha}^{k-2}\left|\nabla u_{\alpha}\right|^{2} d x+\int_{\Omega} h_{\alpha} u_{\alpha}^{k} d x=\int_{\Omega} u_{\alpha}^{q+k-2} d x .
$$


En écrivant

$$
\int_{\Omega} u_{\alpha}^{k-2}\left|\nabla u_{\alpha}\right|^{2} d x=\frac{4}{k^{2}} \int_{\Omega}\left|\nabla u_{\alpha}^{\frac{k}{2}}\right|^{2} d x
$$

et en utilisant l'inégalité de Hölder et (1.6) pour obtenir

$$
\int_{\Omega} u_{\alpha}^{q+k-2} d x \leq\left(\int_{\Omega} u_{\alpha}^{\frac{k q}{2}} d x\right)^{\frac{2}{q}}\left(\int_{\Omega} u_{\alpha}^{q} d x\right)^{\frac{q-2}{q}} \leq \Lambda^{\frac{q-2}{q}}\left(\int_{\Omega} u_{\alpha}^{\frac{k q}{2}} d x\right)^{\frac{2}{q}},
$$

nous en déduisons que

$$
\frac{4(k-1)}{k^{2}}\left\|\nabla u_{\alpha}^{\frac{k}{2}}\right\|_{2}^{2} \leq\left\|h_{\alpha}\right\|_{\infty}\left\|u_{\alpha}\right\|_{k}^{k}+\Lambda^{\frac{q-2}{q}}\left\|u_{\alpha}\right\|_{\frac{k q}{2}}^{k} .
$$

Comme l'injection de $H_{0}^{1}(\Omega)$ dans $L^{2^{\star}}(\Omega)$ est continue, il existe une constante $C>0$ telle que pour toute fonction $\varphi$ dans $H_{0}^{1}(\Omega)$,

$$
\|\varphi\|_{2^{\star}} \leq C\|\nabla \varphi\|_{2} .
$$

En fait, cette constante $C$ est indépendante de $\Omega$ et ne dépend donc que de la dimension $n$. Elle est même explicitement connue, cf. [7] ou [96]. Ainsi nous arrivons à

$$
\frac{4(k-1)}{k^{2}} C^{-2}\left\|u_{\alpha}\right\|_{\frac{2^{\star} k}{2}}^{k} \leq\left\|h_{\alpha}\right\|_{\infty}\left\|u_{\alpha}\right\|_{k}^{k}+\Lambda^{\frac{q-2}{q}}\left\|u_{\alpha}\right\|_{\frac{k q}{2}}^{k} .
$$

Comme, par inégalité de Hölder,

$$
\left\|u_{\alpha}\right\|_{k}^{k} \leq\left\|u_{\alpha}\right\|_{\frac{k q}{2}}^{k} \times \operatorname{Vol}(\Omega)^{\frac{q-2}{q}},
$$

nous obtenons finalement

$$
\left\|u_{\alpha}\right\|_{\frac{2^{\star} k}{2}} \leq\left(C^{2} \frac{k^{2}}{4(k-1)}\right)^{\frac{1}{k}} \times\left(\left\|h_{\alpha}\right\|_{\infty} \operatorname{Vol}(\Omega)^{\frac{q-2}{q}}+\Lambda^{\frac{q-2}{q}}\right)^{\frac{1}{k}}\left\|u_{\alpha}\right\|_{\frac{k q}{2}}
$$

pour tout $\alpha$ et tout $k \geq 2$. Il est aisé d'en déduire, en appliquant ceci successivement à $k_{i}=\left(\frac{2^{\star}}{q}\right)^{i} 2$ pour $i=0,1, \ldots$ qu'il existe une constante $D>0$ ne dépendant que de $\Lambda, h, n, q$ et $\operatorname{Vol}(\Omega)$ telle que

$$
\left\|u_{\alpha}\right\|_{\infty} \leq D
$$

pour tout $\alpha>0$. Le point crucial ici est que $q<2^{\star}$. Dans le cas $q \geq$ $2^{\star}$, l'inégalité ci-dessus ne nous permet pas d'améliorer l'espace $L^{p}$ dans lequel nous pouvons obtenir des bornes uniformes. Il est d'ailleurs sans espoir dans ce cas d'en obtenir sans informations supplémentaires comme le lecteur pourra s'en convaincre à la lecture du chapitre 2. Une fois munie de cette borne uniforme sur la norme $L^{\infty}$ de $u_{\alpha}$, l'équation nous donne 
directement une borne uniforme dans $C^{2, \eta}$ pour tout $\eta>0$ (cf. par exemple le très complet [48] ou le succinct et clair [50] pour toute cette théorie de la régularité elliptique). Une fois cette borne uniforme dans $C^{2, \eta}$ obtenue, le théorème d'Ascoli nous donne l'existence d'une sous-suite de $\left(u_{\alpha}\right)$ qui converge vers une solution de (1.4) par passage à la limite dans (1.5). Mais ceci contredit l'hypothèse (1.7). Ainsi, la proposition est démontrée avec l'hypothèse additionnelle (1.6).

Il nous reste à expliquer comment démontrer la proposition sans cette hypothèse (1.6). Nous avons vu à la fin de la preuve ci-dessus que, si nous avions une borne uniforme $L^{\infty}$ sur une sous-suite quelconque de $\left(u_{\alpha}\right)$, la théorie de la régularité elliptique nous amenait à une contradiction avec (1.7). Ainsi nous pouvons supposer que

$$
\left\|u_{\alpha}\right\|_{\infty} \rightarrow+\infty \text { quand } \alpha \rightarrow+\infty \text {. }
$$

Soit $x_{\alpha} \in \Omega$ tel que $u_{\alpha}\left(x_{\alpha}\right)=\left\|u_{\alpha}\right\|_{\infty}$. Afin de comprendre ce qui se passe au voisinage de $x_{\alpha}$, nous allons procéder à un changement d'échelle en posant

$$
v_{\alpha}(x)=u_{\alpha}\left(x_{\alpha}\right)^{-1} u_{\alpha}\left(x_{\alpha}+\mu_{\alpha} x\right)
$$

pour $x \in \Omega_{\alpha}$ avec

$$
\Omega_{\alpha}=\left\{y \in \mathbb{R}^{n} \text { t.q. } x_{\alpha}+\mu_{\alpha} y \in \Omega\right\} \text {. }
$$

Cette fonction $v_{\alpha}$ atteint alors son maximum, 1 , au point 0 et vérifie

$$
\Delta v_{\alpha}+h_{\alpha}\left(x_{\alpha}+\mu_{\alpha} \cdot\right) \mu_{\alpha}^{2} v_{\alpha}=\mu_{\alpha}^{2} u_{\alpha}\left(x_{\alpha}\right)^{q-2} v_{\alpha}^{q-1}
$$

dans $\Omega_{\alpha}$ avec conditions de Dirichlet au bord. En choisissant $\mu_{\alpha}$ de sorte que

$$
\mu_{\alpha}^{2} u_{\alpha}\left(x_{\alpha}\right)^{q-2}=1
$$

nous avons alors

$$
\Delta v_{\alpha}=v_{\alpha}^{q-1}-h_{\alpha}\left(x_{\alpha}+\mu_{\alpha} \cdot\right) \mu_{\alpha}^{2} v_{\alpha}
$$

dans $\Omega_{\alpha}$. Comme $\left(v_{\alpha}\right)$ est uniformément bornée dans $L^{\infty}\left(\Omega_{\alpha}\right)$ et $\mu_{\alpha} \rightarrow 0$ quand $\alpha \rightarrow+\infty$ (grâce à (1.8)), nous obtenons par théorie de la régularité elliptique standard que $\left(v_{\alpha}\right)$ est uniformément bornée dans $C^{0, \eta}\left(K \cap \Omega_{\alpha}\right)$ pour tout compact $K$ de $\mathbb{R}^{n}$. Ainsi nous pouvons extraire une sous-suite de $v_{\alpha}$ qui converge localement dans $C^{0}$ et passer à la limite dans l'équation pour obtenir

$$
\Delta v=v^{q-1}
$$

dans $\Omega_{\infty}$ où $\Omega_{\infty}$ est soit $\mathbb{R}^{n}$ tout entier, soit un demi-espace auquel cas la condition de Dirichlet est satisfaite au bord. Or la seule solution de $\Delta v=v^{q-1}$ pour $2<q<2^{\star}$ dans $\mathbb{R}^{n}$ entier ou dans un demi-espace avec 
condition de Dirichlet au bord est la solution nulle d'après des résultats de $[11,22,47]$. Mais $v \not \equiv 0$ puisque $v(0)=1$. Nous aboutissons donc à une contradiction, ce qui prouve la proposition dans le cas général.

Dans l'une comme l'autre preuve, il est essentiel que $q<2^{\star}$. Dans la première, en effet, le schème itératif de Moser qui sert à estimer uniformément des normes $L^{p}$ de plus en plus grandes ne peut pas démarrer si $q=2^{\star}$ tandis que, dans la seconde, il existe des solutions de $\Delta v=v^{2^{\star}-1}$ dans $\mathbb{R}^{n}$ tout entier (elles ont d'ailleurs été classifiées par Caffarelli-GidasSpruck [22], cf. chapitre 3). Par contre, dès que $q=2^{\star}$, comme nous le verrons dans la suite, l'étude de la stabilité de (1.4) devient extrêmement intéressante et dépend de la fonction $h$ ainsi que de la géométrie de l'ouvert.

A titre de remarque, une simple adaptation de la preuve ci-dessus permet de montrer que l'équation

$$
\Delta_{g} u+h u=u^{q-1}
$$

est stable sur une variété riemannienne compacte ${ }^{6}$ pour toute fonction $h$ telle que l'opérateur $\Delta_{g}+h$ soit coercif et tout $2<q<2^{\star}$. Dans cette équation, $\Delta_{g}=-\operatorname{div}_{g}(\nabla$.$) est l'opérateur de Laplace-Beltrami. C'est$ d'ailleurs à cette équation pour $q=2^{\star}$ que nous nous intéresserons dans les deux chapitres qui suivent.

6. avec condition de Dirichlet au bord s'il y en a un. 


\section{Chapitre 2}

\section{Etude détaillée d'un exemple : les équations de type Yamabe}

\subsection{Le problème de Yamabe}

En 1960, Yamabe s'est dit qu'il était temps d'essayer de résoudre la conjecture de Poincaré à l'aide d'outils d'analyse ${ }^{1}$. Partons de l'observation d'Hilbert que les métriques d'Einstein sont des points critiques de la fonctionnelle

$$
\mathcal{H}(g)=\operatorname{Vol}_{g}(M)^{-\frac{2}{n}} \int_{M} S_{g} d v_{g}
$$

parmi toutes les métriques sur une variété $M$ de dimension $n$ fixée où $S_{g}$ est la courbure scalaire de $(M, g)$. Poursuivons avec le fait, classique en géométrie riemannienne, qu'une variété compacte simplement connexe de dimension 3 qui admet une métrique d'Einstein est la sphère $S^{3}$. Il en découle qu'une possibilité pour attaquer la conjecture de Poincaré est de trouver un point critique de cette fonctionnelle sur toute variété de dimension 3 compacte simplement connexe. Evidemment, ce ne peut être simple puisque sur certaines variétés, cette fonctionnelle n'admet pas de points critiques : c'est le cas de $S^{1} \times S^{2}$ qui n'admet pas de métrique d'Einstein et donc sur laquelle la fonctionnelle $\mathcal{H}$ n'admet pas de points critiques.

1. Cette intuition s'est trouvée justifiée quelque quarante ans plus tard avec les travaux de Perelman [80, 81, 82], même si ce n'est pas l'angle d'attaque suggéré par Yamabe qui s'est révélé efficace mais celui proposé vingt ans plus tard par Hamilton [49]. 
Comme exercice préparatoire, Yamabe s'est donc proposé de trouver des points critiques de cette fonctionnelle sous la contrainte que la métrique $g$ reste dans une classe conforme fixée. Un tel point critique ne donne plus une métrique d'Einstein mais une métrique à courbure scalaire constante. Trouver une telle métrique peut être intéressant : par exemple, en dimension 2, cela donne le théorème d'uniformisation ${ }^{2}$.

Yamabe affirmait dans son papier [101] de 1960 qu'un tel point critique existait dans toute classe conforme et donc que toute classe conforme possédait une métrique à courbure scalaire constante. Le problème de Yamabe connaît une (re)naissance en 1968 avec la découverte par Trudinger [97] d'une erreur dans le travail de Yamabe [101]. Trudinger, en montrant que la preuve était trouée, allait ouvrir tout un champ d'investigations en analyse géométrique ${ }^{3}$.

Le problème de Yamabe est donc le suivant : étant donnée $(M, g)$ une variété riemannienne compacte ${ }^{4}$, existe-t-il une métrique $\tilde{g}=e^{v} g, v$ fonction régulière de $M$ dans $\mathbb{R}$, qui soit à courbure scalaire constante? En dimension 2 , une réponse positive est une façon de voir le théorème d'uniformisation de Poincaré. En dimensions $n \geq 3$, il vaut mieux poser $e^{v}=u^{\frac{4}{n-2}}$ et les courbures scalaires de $\tilde{g}$ et $g$ sont alors reliées par l'équation

$$
\Delta_{g} u+\frac{n-2}{4(n-1)} S_{g} u=\frac{n-2}{4(n-1)} S_{\tilde{g}} u^{\frac{n+2}{n-2}} .
$$

Résoudre le problème de Yamabe revient donc à trouver un $\lambda \in \mathbb{R}$ et une fonction $u$ régulière et strictement positive solution de

$$
\Delta_{g} u+\frac{n-2}{4(n-1)} S_{g} u=\lambda u^{\frac{n+2}{n-2}} .
$$

Clairement, $\lambda$ peut toujours être supposé appartenir à $\{-1,0,+1\}$. Mais, qui plus est, une fois donnée la variété riemannienne $(M, g)$, le signe de $\lambda$ est déterminé par le signe de la première valeur propre de l'opérateur $\Delta_{g}+\frac{n-2}{4(n-1)} S_{g}$. En effet, si cette première valeur propre est négative (resp. nulle, positive), les seuls $\lambda$ pour lesquels il puisse y avoir une solution à

2. D'aucuns voient le problème de Yamabe comme une extension du théorème d'uniformisation, d'autres expliqueront que c'est le problème de $Q$-courbure en dimension paire. Mais ceci n'a pas grand sens. Le théorème d'uniformisation, une fois qu'il est vu comme problème de prescription de courbure de Gauss constante, peut admettre autant de généralisations qu'il existe de problèmes de prescription de courbure constante puisque la dimension 2 ne présente qu'une seule courbure, celle de Gauss.

3. Il est fort probable que le travail de Pohožaev [83] ait mis la puce à l'oreille de Trudinger. En effet, Pohožaev avait démontré en 1965 qu'une équation similaire à celle pour laquelle Yamabe prétendait avoir trouvé une solution n'en possédait pas nécessairement. Il se trouve d'ailleurs que, dans ces années 60, l'équation en question intéressait à la fois des géomètres et des EDPistes euclidiens.

4. Le problème peut également être posé sur des variétés non compactes. 
l'équation (2.1) sont négatifs (resp. nuls, positifs). Ceci amène à distinguer trois classes de Yamabe : négative, nulle et positive. Dans les cas nul et négatif, il est relativement facile de trouver une solution à l'équation (cf. Trudinger [97]). De plus, la solution est unique dans ces cas-là ${ }^{5}$. Le cas positif a résisté quelques années de plus : il a fallu attendre Aubin [6] en 1976 pour le traitement des variétés de dimensions $n \geq 6$ non localement conformément plates et Schoen [87] en 1984 pour les cas restants, i.e. les dimensions $n \leq 5$ et les variétés localement conformément plates. Un excellent survol sur la résolution du problème de Yamabe a été écrit par Lee et Parker [65]. Ce survol unifie les preuves de Aubin et Schoen en introduisant les coordonnées conformes normales.

Si, lorsque l'invariant de Yamabe est négatif ou nul, il n'y a essentiellement qu'une métrique à courbure scalaire constante, la situation change drastiquement si l'invariant de Yamabe est positif : il existe des variétés riemanniennes à invariant de Yamabe positif pour lesquelles le nombre de métriques à courbure scalaire constante dans la classe conforme de la métrique initiale est arbitrairement grand (voire infini).

Le premier exemple qui vienne à l'esprit est la sphère standard : si $\left(S^{n}, h\right)$ dénote la sphère unité de $\mathbb{R}^{n+1}$ avec la métrique induite de la métrique euclidienne, alors non seulement la métrique standard a une courbure scalaire constante (égale à $n(n-1)$ ) mais une infinité de métriques dans la classe conforme de la métrique standard ont une courbure scalaire constante. Obata [79] a tout d'abord montré que toute métrique à courbure scalaire constante dans la classe conforme de la sphère standard était isométrique à celle-ci. Donc trouver l'ensemble des métriques à courbure scalaire constante dans la classe conforme de la métrique standard revient à trouver l'ensemble des difféomorphismes conformes de la sphère standard, c'est-à-dire des difféomorphismes de la sphère tels que $\varphi^{\star} h$ appartienne à la classe conforme de $h$. Ceci n'est pas difficile une fois remarqué qu'il est possible, quitte à composer par une rotation (isométrie), de fixer un point puis de repasser à un difféomorphisme conforme de l'espace euclidien via projection stéréographique. Nous n'en considérerons qu'une famille, ceux donnés par $\Phi_{P, t}(x)=\pi_{P}\left(t \pi_{P}^{-1}(x)\right)$ où $\pi_{P}$ est la projection stéréographique de pôle $P$ fixé et $t \geq 1$ est un réel. Un simple calcul donne alors

$$
\Phi_{P, t}^{\star} h=u_{P, t}^{\frac{4}{n-2}} h
$$

où

$$
u_{P, t}(x)=\left(\frac{2 t}{1-\cos d_{h}(x, P)+t^{2}\left(1+\cos d_{h}(x, P)\right)}\right)^{\frac{n-2}{2}} .
$$

5. Unique à multiplication par une constante près, ce qui modifie le $\lambda$ dans le cas négatif mais ne le modifie pas dans le cas nul. En particulier, dans une classe conforme à invariant de Yamabe négatif ou nul, il n'existe qu'une seule métrique à courbure scalaire constante de volume 1 . 
Cette fonction vérifie l'équation de Yamabe sur la sphère standard, i.e.

$$
\Delta_{h} u_{P, t}+\frac{n(n-2)}{4} u_{P, t}=\frac{n(n-2)}{4} u_{P, t}^{\frac{n+2}{n-2}} .
$$

Or cette famille à un paramètre de solutions n'est pas bornée dans l'espace $C^{2}$. Elle a une énergie bornée puisque

$$
\operatorname{Vol}_{\Phi_{P, t}^{\star} h}\left(S^{n}\right)=\int_{S^{n}} u_{P, t}^{\frac{2 n}{n-2}} d v_{h}=\omega_{n}=\operatorname{Vol}_{h}\left(S^{n}\right)
$$

pour tout $t \geq 1$. Par contre, il est clair que $\left\|u_{P, t}\right\|_{\infty} \rightarrow+\infty$ quand $t \rightarrow+\infty$ et que la suite $\left(u_{P, t}\right)$ se concentre au point $-P$ lorsque $t \rightarrow+\infty$. C'est le modèle d'explosion qui peut se produire pour une suite de solutions de l'équation de Yamabe.

Revenons à notre problème de multiplicité de métriques à courbure scalaire constante (positive) dans une classe conforme donnée. Hormis la sphère standard, il y a d'autres exemples de multiplicité. Prenons par exemple $M_{T}=S^{n-1} \times S^{1}(T)$ munie de la métrique produit standard. Il est clair que, lorsque $T \rightarrow+\infty$, cette métrique n'atteint plus le mimimum de la fonctionnelle

$$
\left(\int_{M_{T}} S_{g} d v_{g}\right) \operatorname{Vol}_{g}\left(M_{T}\right)^{-\frac{2}{n}}
$$

dans sa classe conforme puisque celui-ci est inférieur à une valeur fixe ne dépendant que de $n$. Or ce minimum est toujours atteint par une métrique à courbure scalaire constante. C'est comme cela que le problème de Yamabe a été résolu par Aubin [6] et Schoen [87]. Ainsi il est clair que, pour un certain $T_{1}$ suffisamment grand, il existe une deuxième métrique à courbure scalaire constante. Cette métrique elle-même se relève naturellement aux revêtements $M_{N T_{1}}$ et cesse d'être minimisante pour $N$ grand. Ainsi, par le même argument, il existe un certain $T_{2}$ pour lequel il existe trois métriques distinctes à courbure scalaire constante dans la classe conforme de la métrique produit. Et ainsi de suite. Cet exemple est dû à Schoen [90] et montre bien qu'il peut, en-dehors de la sphère standard, y avoir une multiplicité de solutions au problème de Yamabe dans une classe conforme donnée ${ }^{6}$. Pollack [84] a d'ailleurs montré que ces exemples étaient nombreux.

\subsection{Compacité}

Dans les deux travaux [89] et [90], Schoen provoqua un coup de tonnerre en annonçant que, excepté sur la sphère standard, l'ensemble des métriques

6. Le résultat dans [90] est plus précis que cela puisqu'il contient un décompte des solutions. Il faut noter que, dans ce cas précis, l'équation de Yamabe se réduit à une équation différentielle ordinaire. 
à courbure scalaire constante dans une classe conforme donnée est précompact dans la topologie $C^{2}$. En termes d'EDP, ceci revient à dire que l'ensemble des solutions de l'équation

$$
\Delta_{g} u+\frac{n-2}{4(n-1)} S_{g} u=u^{\frac{n+2}{n-2}}
$$

est borné a priori dans $C^{2}(M)$, sauf si la variété est conformément difféomorphe à la sphère standard (voir section précédente). Schoen donne une preuve dans le cas conformément plat en supposant une borne a priori sur l'énergie des solutions (ou encore sur le volume des métriques à courbure scalaire constante 1) dans [89]. Dans [90], en s'appuyant sur les travaux de Schoen-Yau [91] et sur l'application développante des variétés localement conformément plates, il donne une preuve particulièrement élégante de ce résultat toujours dans le cas localement conformément plat mais sans borne a priori sur l'énergie des solutions. Il apparaissait clairement que cette deuxième preuve n'avait aucune chance de s'étendre au cas non conformément plat. Mais dans un cours donné à Stanford en 88-89, voir [88], il proposait une approche pour démontrer ce résultat de compacité dans le cas général. Cette approche a été mise en œuvre par Li et Zhu [70] en dimension 3 quelques années plus tard en s'inspirant de SchoenZhang [93] qui avait développé cette analyse dans un cadre proche mais différent. En dimensions plus grandes, le problème apparaissait plus ardu. Un résultat de l'auteur dans [36] le démontre en dimensions 4 et 5 . Puis les choses se sont accélérées puisque Marques [77] l'a ensuite démontré jusqu'à la dimension 7, Li et Zhang [69] jusqu'à la dimension 11 et enfin Khuri, Marques et Schoen [64] l'ont démontré jusqu'à la dimension 24. Il convient de remarquer qu'à partir de la dimension 8 , ces résultats sont conditionnés à la preuve du théorème de la masse positive, disponible seulement en dimensions $n \leq 7$ grâce à [92]. Un autre coup de tonnerre est venu de Brendle [14] puis de Brendle-Marques [16] qui ont démontré que le résultat était faux en dimensions plus grandes ${ }^{7}$.

\subsection{Stabilité - Définitions, exemples}

Nous allons nous intéresser précisément aux questions de stabilité pour une équation de type Yamabe, l'équation

$$
\Delta_{g} u+h u=u^{\frac{n+2}{n-2}}, u \geq 0
$$

7. Brendle [14] trouve des contre-exemples à la compacité en dimensions $n \geq 52$, contre-exemples étendus aux dimensions $25 \leq n \leq 51$ par Brendle et Marques [16]. Le lecteur pourra consulter le récent article de survol de Brendle [15]. 
sur une variété riemannienne compacte $(M, g)$ de dimension $n \geq 3$ où $h$ est une fonction de $M$ dans $\mathbb{R}$ que nous supposerons $C^{1}$ dans la suite ${ }^{8}$. Etant donnés $h \in C^{1}(M)$ et $\Lambda$ un réel strictement positif, nous noterons $\mathcal{S}_{h}^{\Lambda}$ l'ensemble des solutions de l'équation (2.2) d'énergie plus petite que $\Lambda$, i.e.

$$
\mathcal{S}_{h}^{\Lambda}=\left\{u \in C^{2}(M) \text { solution de (2.2) t.q. } \int_{M}|u|^{\frac{2 n}{n-2}} d v_{g} \leq \Lambda\right\} .
$$

Par définition, $\mathcal{S}_{h}^{\infty}$ est l'ensemble des solutions de (2.2).

Définition 2.1. Soit $(M, g)$ une variété riemannienne compacte de dimension $n \geq 3$. Soit $h \in C^{1}(M)$. Soit $\Lambda \in(0,+\infty]$. Nous dirons que l'équation (2.2) est $\Lambda$-stable si pour tout $\varepsilon>0$, il existe $\eta>0$ tel que

$$
\|\tilde{h}-h\|_{C^{1}(M)} \leq \eta \Rightarrow d_{C^{2}}^{\hookrightarrow}\left(\mathcal{S}_{\tilde{h}}^{\Lambda+\varepsilon}, \mathcal{S}_{h}^{\Lambda}\right) \leq \varepsilon
$$

Nous dirons qu'elle est stable si elle est $\Lambda$-stable pour tout $\Lambda>0$. Enfin, si l'équation est $\Lambda$-stable avec $\Lambda=+\infty$, nous dirons qu'elle est globalement stable.

Nous définirons de même la compacité de la façon suivante : l'équation (2.2) sera dite $\Lambda$-compacte si l'ensemble $\mathcal{S}_{h}^{\Lambda}$ est borné dans $C^{2}(M)$. Nous dirons qu'elle est "compacte à énergie bornée" si elle est $\Lambda$-compacte pour tout $\Lambda>0$ et "compacte" si l'ensemble $\mathcal{S}_{h}^{\infty}$ est borné dans $C^{2}(M)$.

Remarque 1 : pour la compacité, pour ne pas aller à l'encontre de la tradition, nous garderons "compacte" pour l' $\infty$-compacité. Par contre, pour la stabilité, il nous semble intéressant d'adopter la terminologie ci-dessus.

Remarque 2 : dans la définition 2.1, un $\varepsilon$ apparaît dans l'énergie des solutions de l'équation perturbée. Il faut en effet pouvoir se permettre, dans ce genre de résultats perturbatifs, de perturber également l'énergie. En général, nous nous intéresserons à la stabilité, pas à la $\Lambda$-stabilité pour un $\Lambda$ fixé. Dans ce cas, ce $\varepsilon$ ne joue bien entendu aucun rôle.

Remarque 3 : il pourrait être intéressant, plutôt que de se donner une borne sur l'énergie pour la stabilité, de se donner une borne sur l'indice de Morse des solutions ${ }^{9}$. Il se trouve que, pour la plupart des équations étudiées ci-dessous, ces deux définitions se révèleraient équivalentes.

8. Une telle régularité n'est pas nécessaire pour nombre de résultats exposés cidessous mais l'objet de ce texte n'est pas de rentrer dans ces détails. Nous renvoyons le lecteur aux textes originaux dont sont issus les résultats pour voir quelles hypothèses de régularité sont nécessaires.

9. Cette définition alternative nous a été proposée par Nassif Ghoussoub. 
Notons tout d'abord que $\mathcal{S}_{h}^{\infty}=\{0\}$ dès que la première valeur propre de l'opérateur $\Delta_{g}+h$ est négative ou nulle ${ }^{10}$. Ainsi, si $\lambda_{1}(h) \leq 0$, il est clair que l'équation $(2.2)$ est compacte. Si $\lambda_{1}(h)<0$, cette propriété est stable par perturbations; aucune équation perturbée ne possède de solutions et donc l'équation (2.2) est globalement stable. Dans la suite, nous supposerons donc que

$$
\lambda_{1}(h)=\inf _{u \in C^{\infty}(M), u \neq 0} \frac{\int_{M}\left(|\nabla u|_{g}^{2}+h u^{2}\right) d v_{g}}{\int_{M} u^{2} d v_{g}} \geq 0 .
$$

Par théorie elliptique standard, une équation est $\Lambda$-compacte s'il existe $C>0$ telle que toute solution $u \in \mathcal{S}_{h}^{\Lambda}$ vérifie $\|u\|_{\infty} \leq C$. Réciproquement, une équation n'est pas $\Lambda$-compacte si il existe une suite de solutions $\left(u_{\alpha}\right)$ dans $\mathcal{S}_{h}^{\Lambda}$ telle que $\left\|u_{\alpha}\right\|_{\infty} \rightarrow+\infty$ quand $\alpha \rightarrow+\infty$.

Toujours par théorie elliptique standard, une équation est $\Lambda$-stable si toute suite de solutions $\left(u_{\alpha}\right)$ d'équations perturbées

$$
\Delta_{g} u_{\alpha}+h_{\alpha} u_{\alpha}=u_{\alpha}^{\frac{n+2}{n-2}} \text { et } \int_{M} u_{\alpha}^{2^{\star}} d v_{g} \leq \Lambda+o(1)
$$

avec $h_{\alpha} \rightarrow h$ dans $C^{1}(M)$ quand $\alpha \rightarrow+\infty$ est uniformément bornée dans $L^{\infty}(M)$. Par contre, la réciproque n'est pas vraie. Elle le sera uniquement si l'équation est $\Lambda$-compacte. Il est seulement vrai qu'une équation $\Lambda$ compacte est $\Lambda$-stable si et seulement si toute suite de solutions $\left(u_{\alpha}\right)$ dans $\mathcal{S}_{h_{\alpha}}^{\Lambda+o(1)}$ avec $h_{\alpha} \rightarrow h$ dans $C^{1}(M)$ quand $\alpha \rightarrow+\infty$ est uniformément bornée dans $L^{\infty}(M)$. Cela soulève une première question :

Question 1 - Existe-t-il des équations qui sont $\Lambda$-stables sans être $\Lambda$ compactes?

Cela nous paraît assez improbable mais tant que ce n'est pas démontré, la notion de stabilité forte définie ci-dessous est pertinente. Si la réponse à la question 1 est non, cette stabilité forte ne sera rien d'autre que la stabilité.

Définition 2.2. Soit $(M, g)$ une variété riemannienne compacte de dimension $n \geq 3$. Soit $h \in C^{1}(M)$. Soit $\Lambda \in(0,+\infty)$. Nous dirons que l'équation (2.2) est fortement $\Lambda$-stable si elle est $\Lambda$-compacte et $\Lambda$-stable. Nous dirons qu'elle est fortement stable si elle est fortement $\Lambda$-stable pour tout $\Lambda>0$. Pour $\Lambda=+\infty$, nous dirons alors qu'elle est globalement fortement stable.

10. En effet, en testant l'équation (2.2) contre la fonction propre $\varphi$ strictement positive associée à la première valeur propre $\lambda_{1}$ de $\Delta_{g}+h$, nous obtenons $\int_{M} u^{\frac{n+2}{n-2}} \varphi d v_{g}=\lambda_{1} \int_{M} u \varphi d v_{g}$, ce qui impose $\lambda_{1}>0$ si $u \not \equiv 0$. 
La question 1 revient à poser la question de l'équivalence entre $\Lambda$ stabilité et forte $\Lambda$-stabilité. Considérons maintenant une fonction $h \in$ $C^{1}(M)$ pour laquelle l'équation (2.2) correspondante n'est pas fortement $\Lambda$-stable. Ceci signifie qu'il existe une suite $\left(h_{\alpha}\right)$ de fonctions $C^{1}$ sur $M$ telle que $h_{\alpha} \rightarrow h$ dans $C^{1}(M)$ quand $\alpha \rightarrow+\infty$ et une suite de solutions strictement positives $\left(u_{\alpha}\right)$ de l'équation

$$
\Delta_{g} u_{\alpha}+h_{\alpha} u_{\alpha}=u_{\alpha}^{\frac{n+2}{n-2}}
$$

avec

$$
\int_{M} u_{\alpha}^{\frac{2 n}{n-2}} d v_{g} \leq \Lambda+o(1)
$$

qui n'est pas uniformément bornée dans $L^{\infty}(M)$. Si $\Lambda=+\infty$, cette dernière condition est vide. Si $\Lambda<+\infty$, grâce à la décomposition $H_{1}^{2}$ de Struwe [94], cf. chapitre 3 ci-dessous, il existe $N \in \mathbb{N}^{\star}$ tel que

$$
u_{\alpha}=u_{0}+\sum_{i=1}^{N} B_{i, \alpha}+R_{\alpha}
$$

où $R_{\alpha} \rightarrow 0$ dans $H_{1}^{2}(M)$ quand $\alpha \rightarrow+\infty$, les $B_{i, \alpha}$ sont des bulles standards de centre $\left(x_{i, \alpha}\right)$ et de poids $\left(\mu_{i, \alpha}\right)$ et $u_{0} \in \mathcal{S}_{h}^{\infty}$. Ces bulles sont de la forme

$$
B_{i, \alpha}=\mu_{i, \alpha}^{\frac{n-2}{2}}\left(\mu_{i, \alpha}^{2}+\frac{d_{g}\left(x_{i, \alpha}, x\right)^{2}}{n(n-2)}\right)^{1-\frac{n}{2}}
$$

où $\left(x_{i, \alpha}\right)$ est une suite de points de $M$, appelés centre de la bulle, et $\left(\mu_{i, \alpha}\right)$ est une suite de réels strictement positifs tendant vers 0 lorsque $\alpha \rightarrow+\infty$, appelés poids de la bulle. L'énergie d'une bulle tend vers une constante universelle lorsque $\alpha$ tend vers $+\infty$ :

$$
\int_{M} B_{i, \alpha}^{2^{\star}} d v_{g}=E_{n}+o(1)
$$

où

$$
E_{n}=\left(\frac{n(n-2)}{4}\right)^{\frac{n}{2}} \omega_{n}
$$

De plus,

$$
\Lambda+o(1) \geq \int_{M} u_{\alpha}^{\frac{2 n}{n-2}} d v_{g}=\int_{M} u_{0}^{\frac{2 n}{n-2}} d v_{g}+N E_{n}+o(1) .
$$

On en déduit que $u_{0} \in \mathcal{S}_{h}^{\Lambda-N E_{n}}$. Il s'ensuit en particulier que l'équation (2.2) est toujours fortement $\Lambda$-stable si $\Lambda<E_{n}$. 
L'équation de Yamabe sur la sphère standard, i.e. avec $h \equiv \frac{n(n-2)}{4}$ n'est pas $\Lambda$-compacte dès lors que $\Lambda \geq E_{n}$. Elle n'est d'ailleurs sans doute pas $\Lambda$-stable non plus (cf. discussion de la question 1).

L'équation de Yamabe sur une variété riemannienne compacte $(M, g)$, i.e. avec $h \equiv \frac{n-2}{4(n-1)} S_{g}$, est compacte si la dimension de la variété est inférieure ou égale à 24 ou si la variété est localement conformément plate (et n'est pas conformément difféomorphe à la sphère standard). Par contre, dès que la variété est localement conformément plate, cette équation n'est pas $E_{n}$-stable (cf. [38]). Et il existe des exemples de variétés de dimension $n \geq 25$ sur lesquelles elle n'est pas $E_{n}$-compacte.

Ces quelques exemples montrent que cette notion de stabilité est riche, plus riche que la notion de compacité : il existe par exemple des équations compactes instables.

\subsection{Résultats de stabilité et d'instabilité}

Nous allons commencer par décrire un résultat de stabilité obtenu dans [35]. C'est lui qui va ouvrir tout un champ de questions autour de cette notion. Le résultat est le suivant :

Théorème 2.1. [Druet, [35]] Soit $(M, g)$ une variété riemannienne compacte de dimension $n \geq 4$. Soit $h \in C^{1}(M)$ telle que l'opérateur $\Delta_{g}+h$ soit coercif. Si $h(x) \neq \frac{n-2}{4(n-1)} S_{g}(x)$ pour tout $x \in M$ et si $n \neq 6$, alors l'équation (2.2) est fortement stable.

En d'autres termes, sous les hypothèses du théorème, pour tout $\Lambda>0$, pour tout $\varepsilon>0$, il existe $\delta>0$ tel que

$$
\|\tilde{h}-h\|_{C^{1}(M)} \leq \delta \Longrightarrow d_{C^{2}}^{\hookrightarrow}\left(\mathcal{S}_{\tilde{h}}^{\Lambda+\varepsilon}, \mathcal{S}_{h}^{\Lambda}\right) \leq \varepsilon
$$

Ainsi nous voyons que l'équation de Yamabe est critique à bien des égards. Elle l'est bien sûr à cause de la non-linéarité en $u^{\frac{n+2}{n-2}}$ qui correspond au cas limite des injections de Sobolev mais elle l'est également du point de vue de son potentiel. Dès lors que le potentiel $h$ ne touche pas le potentiel de l'équation de Yamabe, l'équation (2.2) est fortement stable excepté en dimensions 3 et 6 . Rappelons que, pour le cas de l'équation de Yamabe elle-même, la forte stabilité (et même la stabilité) est fausse sur certaines variétés de grande dimension et sur la sphère standard en toutes dimensions.

Notons enfin que le résultat resterait vrai si on perturbait la métrique dans des espaces suffisamment réguliers. Pour des résultats d'instabilité ou plutôt de non-compacité - de l'équation de Yamabe elle-même lorsqu'on 
casse suffisamment la régularité de la métrique, nous renvoyons à BertiMalchiodi [12].

Nous ne dirons que quelques mots de la preuve maintenant, afin d'introduire un certain nombre de notations et de notions intéressantes pour la suite de ce chapitre. Mais nous décrirons dans ces grandes lignes la preuve du théorème 2.1 dans le chapitre suivant ${ }^{11}$.

Elle procède par l'absurde. Supposons que, sur une variété riemannienne compacte $(M, g)$ de dimension $n \geq 3$, nous ayons une suite $\left(u_{\alpha}\right)$ de solutions $C^{2}$ aux problèmes suivants :

$$
\left\{\begin{array}{l}
\Delta_{g} u_{\alpha}+h_{\alpha} u_{\alpha}=u_{\alpha}^{\frac{n+2}{n-2}} \\
u_{\alpha}>0 \text { dans } M \\
\int_{M} u_{\alpha}^{\frac{2 n}{n-2}} d v_{g} \leq \Lambda
\end{array}\right.
$$

où $\left(h_{\alpha}\right)$ est une suite de fonctions $C^{1}$ telle que $h_{\alpha} \rightarrow h$ dans $C^{1}(M)$ quand $\alpha \rightarrow+\infty$. Supposons de plus que $\lambda_{1}(h)>0$. Si l'équation (2.2) n'est pas fortement stable, il existe une telle suite de solutions $\left(u_{\alpha}\right)$ qui n'est pas uniformément bornée dans $L^{\infty}(M)$. Nous supposerons donc que c'est le cas et que $\sup u_{\alpha} \rightarrow+\infty$ quand $\alpha \rightarrow+\infty$. La suite $\left(u_{\alpha}\right)$ développe des points de concentration et nous avons, après extraction d'une sous-suite,

$$
u_{\alpha}=u_{0}+\sum_{i=1}^{N} B_{i, \alpha}+R_{\alpha}
$$

avec $R_{\alpha} \rightarrow 0$ dans $H_{1}^{2}(M)$ quand $\alpha \rightarrow+\infty, u_{0}$ solution - soit strictement positive soit identiquement nulle par principe du maximum - de l'équationlimite, $N$ un entier et les $B_{i, \alpha}$ des bulles standard données par

$$
B_{i, \alpha}(x)=\mu_{i, \alpha}^{\frac{n-2}{2}}\left(\mu_{i, \alpha}^{2}+\frac{d_{g}\left(x_{i, \alpha}, x\right)^{2}}{n(n-2)}\right)^{1-\frac{n}{2}}
$$

pour des suites $\left(x_{i, \alpha}\right)$ de points de $M$ et $\left(\mu_{i, \alpha}\right)$ de réels strictement positifs tendant vers 0 lorsque $\alpha \rightarrow+\infty$. Nous renvoyons au chapitre 3 pour des détails. De plus, nous noterons $x_{i}=\lim _{\alpha \rightarrow+\infty} x_{i, \alpha}$, qui existent quitte à extraire encore une sous-suite. Le théorème 3.1 nous donne même une description ponctuelle de la suite $\left(u_{\alpha}\right)$ :

$u_{\alpha}\left(x_{\alpha}\right)=(1+o(1)) u_{0}\left(x_{\alpha}\right)+\sum_{i=1}^{N}(1+o(1)) B_{i, \alpha}\left(x_{\alpha}\right)+O\left(\left(\max _{i=1, \ldots, N} \mu_{i, \alpha}\right)^{\frac{n-2}{2}}\right)$

11. Le lecteur intéressé par la preuve d'un tel résultat peut bien sûr aller consulter le chapitre 3 avant de poursuivre la lecture du présent chapitre. 
pour toute suite $\left(x_{\alpha}\right)$ de points de $M$. Il reste à comprendre pourquoi ce n'est pas possible si le potentiel $h$ ne touche pas le potentiel de l'équation de Yamabe. L'idée est qu'un des points de concentration devra se trouver en un point où $h(x)=\frac{n-2}{4(n-1)} S_{g}(x)$, tout du moins si la dimension n'est ni 3 ni 6 . Ce point sera celui correspondant au poids $\mu_{i, \alpha}$ le plus grand, c'est-à-dire correspondant à la bulle la moins haute et la plus étalée. Toute la difficulté provient du fait que cette bulle risque fortement d'être cachée par d'autres bulles plus hautes. Il faut donc aller la chercher. Comment le faire, ainsi que la preuve de l'estimée ponctuelle ci-dessus, sera expliqué dans le chapitre 3 .

Nous pouvons même obtenir des renseignements très précis sur les points de concentration $x_{i}$ et sur les $u_{0}$ qui peuvent apparaître dans la description (2.4) d'une suite de solutions de (2.3). En particulier, nous savons que :

- si $3 \leq n \leq 5$, alors $u_{0} \equiv 0$.

- si $n=4,5$, il existe au moins un point de concentration $x_{i}$ pour lequel $h\left(x_{i}\right)=\frac{n-2}{4(n-1)} S_{g}\left(x_{i}\right)$.

- si $n \geq 7$, tous les points de concentration $x_{i}$ sont tels que $h\left(x_{i}\right)=$ $\frac{n-2}{4(n-1)} S_{g}\left(x_{i}\right)$.

- si $n=6$ et si $u_{0} \equiv 0$, nous sommes dans le cas des dimensions $n \geq 7$.

- si $n=6$ et si $u_{0} \not \equiv 0$, alors tous les points de concentration $x_{i}$ sont tels que $h\left(x_{i}\right)>\frac{n-2}{4(n-1)} S_{g}\left(x_{i}\right)$.

Ce résultat ouvre la porte à de nombreuses questions autour de cette notion de stabilité des équations de type Yamabe, questions sur lesquelles nous reviendrons dans la section suivante.

Avant cela, nous allons discuter des hypothèses du théorème 2.1. Tout d'abord, il y est démontré un résultat de stabilité forte. Une question naturelle est : cette stabilité est-elle globale? En d'autres termes, peuton s'affranchir de la borne sur l'énergie dans ce résultat. Ce n'est pas si simple et la preuve en est en tout cas complètement différente ${ }^{12}$. Nous avons obtenu dans [36], le cas $n=3$ étant dû̀ à Li-Zhu [70] :

Théorème 2.2. [Druet, [36]] Soit $(M, g)$ une variété riemannienne compacte de dimension $n \geq 3$. L'équation (2.2) est globalement fortement stable dès que $h<\frac{n-2}{4(n-1)} S_{g}$ sur $M$.

Ce théorème amène une question naturelle, sans aucun doute extrêmement délicate :

Question 2 - L'équation (2.2) est-elle globalement fortement stable si $h_{0}>\frac{n-2}{4(n-1)} S_{g}$ en dimensions $n \neq 3,6 ?$

12. cf. section 3.5 pour une discussion des différences. 
Il est à peu près certain que la réponse est non sans hypothèses supplémentaires. Le but serait plutôt de construire des exemples de $h_{0}$ pour lesquels la réponse est négative, éventuellement de trouver des conditions pour que la réponse soit positive; l'idéal serait bien entendu d'obtenir une condition nécessaire et suffisante, ou au moins de s'en approcher.

Il serait très agréable également de comprendre quel rôle joue le noyau de l'opérateur $\Delta_{g}+h$ s'il y en a un. Nous avons vu que la première valeur propre de cet opérateur doit être positive ou nulle pour que la question de la stabilité ait un intérêt. Si celle-ci est nulle, le noyau de cet opérateur est 1-dimensionnel. Quid de la stabilité dans ce cas?

Question 3 - Est-il possible d'obtenir une caractérisation des équations fortement stables lorsque $\lambda_{1}(h)=0$ ?

Comme nous le verrons section 3.2, la présence du noyau influence fortement le profil des suites de solutions qui explosent et son rôle dans cette question de stabilité n'est pas encore clair.

\subsection{Lieux d'instabilité}

Etant donnée une équation (2.2) qui n'est pas fortement $\Lambda$-stable, $\Lambda$ fini, et une suite $\left(u_{\alpha}\right)$ satisfaisant $(2.3)$ qui explose, il existe un certain nombre de points, les limites des centres des bulles $x_{i, \alpha}$ dans la décomposition (2.4), tels que, à extraction près,

$$
u_{\alpha} \rightarrow u_{0} \text { dans } C^{2}\left(M \backslash\left\{x_{i}\right\}_{i=1, \ldots, N}\right) \text { quand } \alpha \rightarrow+\infty .
$$

Nous renvoyons encore une fois au chapitre 3 pour des détails. Nous appellerons cet ensemble de points où la convergence n'a pas lieu $\mathcal{C}\left(u_{\alpha}\right)$. Cet ensemble dépend de la suite considérée (et en fait même de la soussuite extraite). Ainsi il n'est pas très bien défini. Ce qui est bien défini par contre, c'est l'ensemble de ces points pour toutes les suites $\left(u_{\alpha}\right)$ possibles. Posons

$$
\mathcal{C}_{h}^{\Lambda}=\bigcup_{\left(u_{\alpha}\right)} \mathcal{C}\left(u_{\alpha}\right)
$$

où l'union est prise sur l'ensemble des suites de solutions de (2.3) vérifiant (2.5). En d'autres termes, un point $x \in M$ est dans $\mathcal{C}_{h}^{\Lambda}$ si et seulement si il existe une suite $\left(u_{\alpha}\right)$ de solutions de $(2.3)$ avec $h_{\alpha} \rightarrow h$ dans $C^{1}(M)$ qui vérifie (2.5) et où l'un des $x_{i}$ est le point $x$ en question. L'ensemble $\mathcal{C}_{h}^{\Lambda}$ sera dit "ensemble de points d'instabilité" de l'équation (2.2). Si celui-ci est vide, alors l'équation (2.2) est fortement $\Lambda$-stable. 
Le théorème 2.1 ou plus exactement l'analyse faite dans [35] nous dit que

$$
\mathcal{C}_{h}^{\Lambda} \subset\left\{x \in M \text { t.q. } h(x)=\frac{n-2}{4(n-1)} S_{g}(x)\right\}
$$

dès que la dimension n'est ni 3 ni 6 . En dimension 6 , nous avons en fait

$$
\mathcal{C}_{h}^{\Lambda} \subset\left\{x \in M \text { t.q. } h(x)=\frac{1}{5} S_{g}(x)+2 u(x) \text { pour un } u \in \mathcal{S}_{h}^{\Lambda-E_{n}}\right\} .
$$

Une question assez naturelle est la suivante :

Question 4 - Etant donnée une fonction $h \in C^{1}(M)$ et $\Lambda \in \mathbb{R}^{+}$, peut-on caractériser exactement $\mathcal{C}_{h}^{\Lambda}$ ? Et bien entendu en déduire des conditions nécessaires et suffisantes sur $h$ pour que l'équation (2.2) soit fortement stable?

Hormis les informations ci-dessus, nous savons que cet ensemble est vide dès que $\Lambda<E_{n}$. En dimensions $n \geq 7$, nous pouvons conjecturer sans grand risque que

$$
\mathcal{C}_{h}^{\Lambda}=\mathcal{F}(h)
$$

pour tout $\Lambda \geq E_{n}$ où

$$
\begin{aligned}
& \mathcal{F}(h)=\{x \in M \text { t.q. }\left|h(x)-\frac{n-2}{4(n-1)} S_{g}(x)\right| \\
&\left.+\left|\nabla\left(h-\frac{n-2}{4(n-1)} S_{g}\right)(x)\right|_{g}=0\right\} .
\end{aligned}
$$

En dimensions $4 \leq n \leq 6$, une caractérisation complète semble plus délicate. Il devrait cependant être possible de démontrer que $\mathcal{F}(h) \subset \mathcal{C}_{h}^{\Lambda}$ dès que $\Lambda \geq E_{n}$. Ensuite, cet ensemble de points d'instabilité devrait dépendre de $\Lambda$. En particulier, il semble qu'une fois que $\Lambda \geq 2 E_{n}$, il soit en dimensions 4 et 5 beaucoup plus gros que $\mathcal{F}(h)$.

Enfin, nous pouvons nous intéresser aux limites faibles de suites de solutions de (2.3) qui explosent. En dimensions 3, 4 et 5, une suite de solutions $\left(u_{\alpha}\right)$ qui explose ne peut avoir une limite faible que nulle. Il est impossible de développer des points de concentration avec une limite faible nonnulle. Notons $\mathcal{C} S_{h}^{\Lambda}$ l'ensemble des fonctions $u \in \mathcal{S}_{h}^{\infty}$ qui peuvent apparaître comme limite faible d'une suite de solutions $\left(u_{\alpha}\right)$ de $(2.3)$ qui explose. Cet ensemble est l'ensemble des solutions de (2.2) cause d'instabilité. En dimensions $3 \leq n \leq 5$, comme démontré dans [35], $\mathcal{C} S_{h}^{\Lambda}=\{0\}$. Il est naturel de se demander quel est cet ensemble de solutions cause d'instabilité en dimensions plus grandes :

Question 5 - Est-il possible de caractériser $\mathcal{C} S_{h}^{\Lambda}$ en dimensions $n \geq 6$ ? 
En dimensions $n \geq 7$, la réponse devrait être

$$
\mathcal{C} S_{h}^{\Lambda}=\mathcal{S}_{h}^{\Lambda-E_{n}}
$$

Autant dire qu'en dimensions $n \geq 7$, hormis la contrainte triviale que la solution doit avoir une énergie plus petite que $\Lambda-E_{n}$, il ne devrait pas y en avoir d'autres. En dimension $n=6$, la situation est plus compliquée.

\subsection{Et si les solutions changent de signe?}

L'univers des solutions changeant de signe est totalement différent. Pour obtenir des résultats de stabilité, il faut absolument, pour l'instant, repasser dans l'espace euclidien (ou sur des variétés localement conformément plates) afin d'utiliser les symétries de celui-ci. La raison en est la suivante : l'analyse asymptotique décrite au chapitre 3 pour les suites de solutions positives n'a pas d'analogue pour des solutions changeant de signe. Mentionnons le résultat suivant dans le cas conformément plat :

Théorème 2.3 (Vétois, [98]). Soit $(M, g)$ une variété riemannienne localement conformément plate de dimension $n$. L'équation

$$
\Delta_{g} u+h u=|u|^{\frac{4}{n-2}} u
$$

est fortement stable (mais pas fortement globalement stable) en dimensions $n \geq 7$ si $h<\frac{n-2}{4(n-1)} S_{g}$.

Il faut comparer ce résultat au théorème 2.2 qui stipulait que la forte stabilité globale était vraie sous les hypothèses du théorème dans le monde des solutions positives. Le cas euclidien du théorème ci-dessus avait été étudié auparavant par Devillanova et Solimini [30, 31]. 


\section{Chapitre 3}

\section{Analyse asymptotique - un outil pour la stabilité}

\subsection{Description ponctuelle des défauts de compacité}

Dans [42], nous nous sommes attachés avec E. Hebey et F. Robert à décrire les défauts de compacité pour des équations de type Yamabe dans des espaces ponctuels, ce qui constitue un saut qualitatif au-delà des espaces d'énergie. Si cette théorie a été développée en toute généralité pour des équations modelées sur l'équation de Yamabe, les idées et le schéma général de preuve ont déjà été appliqués à des équations différentes (cf. chapitre 4) et sont sûrement voués à servir dans beaucoup de problèmes. Pour aborder les questions de stabilité, cette théorie constitue un outil fondamental.

Considérons une variété riemannienne compacte $(M, g)$ de dimension $n \geq 3$ et considérons l'équation

$$
\Delta_{g} u+h u=u^{\frac{n+2}{n-2}}
$$

où $h$ est une fonction de $M$ dans $\mathbb{R}$ régulière ${ }^{1}$ et $\Delta_{g}$ est le laplacien. La non-linéarité $u^{\frac{n+2}{n-2}}$ est critique et nous n'avons a priori aucune chance de contrôler un tant soit peu l'ensemble des solutions de cette équation.

Etant donnée une suite de solutions positives $\left(u_{\alpha}\right)$ d'équations

$$
\Delta_{g} u_{\alpha}+h_{\alpha} u_{\alpha}=u_{\alpha}^{\frac{n+2}{n-2}},
$$

1. Nous préciserons ultérieurement quelle régularité est requise en fonction des résultats. 
l'objectif est de décrire les comportements possibles de cette suite. Dans l'espace d'énergie associé à l'équation, i.e. $H_{1}^{2}(M)$, cela a été fait par Struwe [94] (cf. le chap. 4 de [42] pour une preuve détaillée dans le cas riemannien). Supposons que la suite $\left(h_{\alpha}\right)$ converge dans $L^{2}(M)$. Supposons également que la suite $\left(u_{\alpha}\right)$ soit d'énergie bornée, c'est-à-dire qu'il existe $\Lambda$ tel que $\int_{M}\left|\nabla u_{\alpha}\right|_{g}^{2} d v_{g} \leq \Lambda$ pour tout $\alpha$. Alors, à extraction d'une sous-suite près, la suite $\left(u_{\alpha}\right)$ peut s'écrire sous la forme

$$
u_{\alpha}=u_{0}+\sum_{i=1}^{N} B_{i, \alpha}+R_{\alpha}
$$

où $u_{0}$ est une solution (éventuellement nulle) de l'équation limite

$$
\Delta_{g} u_{0}+h_{0} u_{0}=u_{0}^{\frac{n+2}{n-2}}
$$

les $B_{i, \alpha}$ sont des "bulles standard" et $R_{\alpha}$ est un reste qui tend vers 0 dans $H_{1}^{2}(M)$. Les bulles standard sont de la forme

$$
B_{i, \alpha}=\mu_{i, \alpha}^{\frac{n-2}{2}}\left(\mu_{i, \alpha}^{2}+\frac{d_{g}\left(x_{i, \alpha}, x\right)^{2}}{n(n-2)}\right)^{1-\frac{n}{2}}
$$

où $\left(x_{i, \alpha}\right)$ est une suite de points de $M$, appelés centre de la bulle, et $\left(\mu_{i, \alpha}\right)$ est une suite de réels strictement positifs tendant vers 0 lorsque $\alpha \rightarrow+\infty$, appelés poids de la bulle. Ces bulles sont modelées sur les fonctions extrémales de l'inégalité de Sobolev euclidienne. En particulier, si la variété considérée était l'espace euclidien, nous aurions exactement $\Delta_{\xi} B_{i, \alpha}=B_{i, \alpha}^{\frac{n+2}{n-2}}$. Ici, nous avons

$$
\Delta_{g} B_{i, \alpha}=B_{i, \alpha}^{\frac{n+2}{n-2}}+R_{i, \alpha}
$$

où $R_{i, \alpha}$ est un terme d'erreur qui tend vers 0 dans $H_{1}^{2}(M)$. L'apparition de ces bulles est la trace du défaut de compacité inhérent à l'équation. En effet, si $N=0$, on peut alors montrer, quitte à supposer une convergence de la suite $\left(h_{\alpha}\right)$ dans des espaces plus réguliers, que la suite $\left(u_{\alpha}\right)$ est uniformément bornée dans $C^{2}(M)$. Par contre, dès que $N \geq 1$, il est clair que la suite $\left(u_{\alpha}\right)$ ne peut plus être uniformément bornée ponctuellement; elle perd même de l'énergie à la limite. L'énergie d'une bulle tend vers une constante universelle lorsque $\alpha$ tend vers $+\infty$ :

$$
\int_{M}\left|\nabla B_{i, \alpha}\right|_{g}^{2} d v_{g}=\left(\frac{n(n-2)}{4}\right)^{\frac{n}{2}} \omega_{n}+o(1)=E_{n}+o(1) .
$$

De plus, dans la décomposition (3.1) de $u_{\alpha}$, les bulles n'interagissent pas au niveau $H_{1}^{2}$. En d'autres termes,

$$
\int_{M}\left(\nabla B_{i, \alpha}, \nabla B_{j, \alpha}\right)_{g} d v_{g} \rightarrow 0 \text { quand } \alpha \rightarrow+\infty
$$


dès que $i \neq j$. Ceci se traduit par des relations entre les poids des bulles et la distance entre leurs centres respectifs :

$$
\frac{d_{g}\left(x_{i, \alpha}, x_{j, \alpha}\right)^{2}}{\mu_{i, \alpha} \mu_{j, \alpha}}+\frac{\mu_{i, \alpha}}{\mu_{j, \alpha}}+\frac{\mu_{j, \alpha}}{\mu_{i, \alpha}} \rightarrow+\infty \text { quand } \alpha \rightarrow+\infty
$$

pour tous $i, j \in\{1, \ldots, N\}, i \neq j$. La perte d'énergie par passage à la limite est donc quantifiée puisque

$$
\lim _{\alpha \rightarrow+\infty} \int_{M}\left|\nabla u_{\alpha}\right|_{g}^{2} d v_{g}=\int_{M}\left|\nabla u_{0}\right|_{g}^{2} d v_{g}+N E_{n}
$$

Le cas $N=0$ correspond à une convergence forte de $\left(u_{\alpha}\right)$ vers sa limite $u_{0}$ tandis que, dès que $N \geq 1$, la suite $\left(u_{\alpha}\right)$ converge faiblement mais pas fortement vers $u_{0}$.

En fait, la décomposition (3.1) a lieu dans un cadre beaucoup plus général puisqu'il suffit que la suite $\left(u_{\alpha}\right)$ soit une suite de Palais-Smale pour les fonctionnelles

$$
J_{\alpha}(u)=\frac{1}{2} \int_{M}\left(\left|\nabla u_{\alpha}\right|_{g}^{2}+h_{\alpha} u_{\alpha}^{2}\right) d v_{g}-\frac{n-2}{2 n} \int_{M}\left|u_{\alpha}\right|^{\frac{2 n}{n-2}} d v_{g} .
$$

En d'autres termes, il suffit que la suite $\left(u_{\alpha}\right)$ soit une suite de fonctions positives ou nulles dans $H_{1}^{2}(M)$ vérifiant

$$
\int_{M}\left(\nabla u_{\alpha}, \nabla \varphi_{\alpha}\right)_{g} d v_{g}+\int_{M} h_{\alpha} u_{\alpha} \varphi_{\alpha} d v_{g}=\int_{M} u_{\alpha}^{\frac{n+2}{n-2}} \varphi_{\alpha} d v_{g}+o(1)
$$

pour toute suite de fonctions $\left(\varphi_{\alpha}\right)$ de $H_{1}^{2}(M)$ telles que $\left\|\varphi_{\alpha}\right\|_{H_{1}^{2}(M)}=1$.

Si une bulle apparaît dans la décomposition (3.1), nous dirons que la suite $\left(u_{\alpha}\right)$ développe un phénomène de concentration. Nous appellerons les suites $\left(x_{i, \alpha}\right)$ des points de concentration et les suites $\left(\mu_{i, \alpha}\right)$ leur poids (ou vitesse de concentration) respectif. Ainsi, en un sens $H_{1}^{2}$, la suite $\left(u_{\alpha}\right)$ ressemble à la somme de sa limite faible et d'un certain nombre de bulles (cf. fig. 1, page 33).

La question qui se pose naturellement et à laquelle nous allons répondre dans cette partie est alors : est-il possible d'obtenir une description similaire dans des espaces ponctuels? Passer d'une description dans l'espace d'énergie $H_{1}^{2}$ à une description ponctuelle dans l'espace $C^{0}$ représente un saut qualitatif énorme. La difficulté essentielle pour passer d'une description à l'autre est que les bulles, si elles n'interagissent pas dans les espaces d'énergie, peuvent très bien interagir au niveau ponctuel. Il n'est par exemple certainement pas vrai que

$$
\left(B_{i, \alpha}+B_{j, \alpha}\right)^{\frac{2 n}{n-2}}-B_{i, \alpha}^{\frac{2 n}{n-2}}-B_{j, \alpha}^{\frac{2 n}{n-2}} \rightarrow 0 \text { dans } C^{0}(M) \text { quand } \alpha \rightarrow+\infty
$$


alors que cette convergence a lieu dans $L^{1}(M)$, ce qui correspond à l'espace d'énergie. Ceci peut être illustré par la figure 2, page 33. Les bulles peuvent même se superposer les unes aux autres sans interagir au niveau $H_{1}^{2}$ : elles peuvent avoir même centre sans violer la relation (3.3); il suffit que l'une d'elles soit beaucoup plus concentrée que l'autre (cf. figure 3, page 34).

Dans un grand nombre d'applications, il est particulièrement intéressant de comprendre ces mécanismes d'interaction. En particulier, si interaction ponctuelle il y a, celle-ci obéit à un certain nombre de contraintes. La machinerie nécessaire pour décrire ces phénomènes de concentration a été élaborée sur le cas des équations de type Yamabe dans le livre [42]. Elle s'adapte parfaitement bien à un certain nombre de problèmes présentant des défauts de compacité classifiables (cf. par exemple [37, 41, 45, 95] ou le chapitre 4). Le résultat démontré dans [42] est le suivant :

Théorème 3.1. [Druet-Hebey-Robert, [42]] Soit $(M, g)$ une variété riemannienne compacte de dimension $n \geq 3$. Soit $\left(h_{\alpha}\right)$ une suite de fonctions dans $C^{0, \eta}(M)$ pour un certain $0<\eta<1$ qui converge vers une fonction limite $h_{0}$ dans $C^{0, \eta}(M)$ lorsque $\alpha \rightarrow+\infty$. Considérons une suite de solutions strictement positives $\left(u_{\alpha}\right)$ de

$$
\Delta_{g} u_{\alpha}+h_{\alpha} u_{\alpha}=u_{\alpha}^{\frac{n+2}{n-2}}
$$

sur $M$ d'énergie a priori bornée $\int_{M}\left|\nabla u_{\alpha}\right|_{g}^{2} d v_{g} \leq \Lambda$. Supposons également que l'opérateur $\Delta_{g}+h_{0}$ a un noyau trivial.

Alors il existe $u_{0} \in C^{2}(M)$ solution (éventuellement nulle) de l'équation limite $\Delta_{g} u_{0}+h_{0} u_{0}=u_{0}^{\frac{n+2}{n-2}}$ et $N$ bulles $B_{i, \alpha}$ de la forme (3.2) telles que, après extraction d'une sous-suite,

$u_{\alpha}\left(x_{\alpha}\right)=(1+o(1)) u_{0}\left(x_{\alpha}\right)+\sum_{i=1}^{N}(1+o(1)) B_{i, \alpha}\left(x_{\alpha}\right)+O\left(\left(\max _{i=1, \ldots, N} \mu_{i, \alpha}\right)^{\frac{n-2}{2}}\right)$

pour toute suite de points $\left(x_{\alpha}\right)$. 


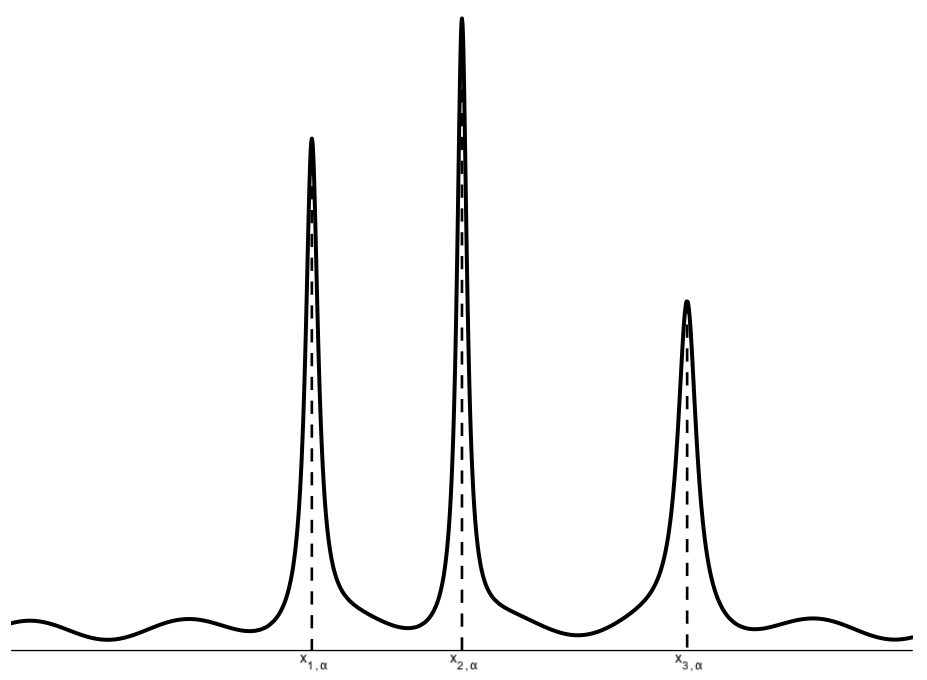

Fig. $1: u_{0}+B_{1, \alpha}+B_{2, \alpha}+B_{3, \alpha}$

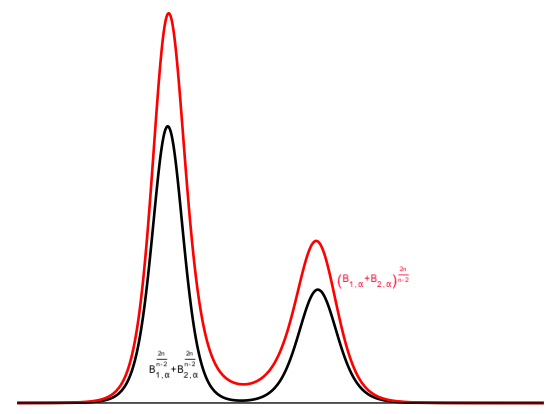

Fig. 2 : Interaction ponctuelle de bulles. 


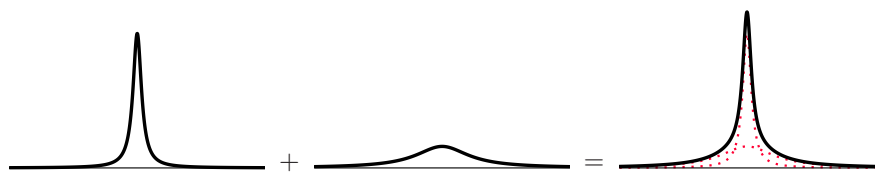

Fig. 3 : une bulle + une bulle $=$ une bulle sur une bulle (même centre).

Ce théorème répond parfaitement à la question de la description ponctuelle de la suite posée ci-dessus. Une fois la description dans l'espace $C^{0}$ obtenue, les effets de régularisation des EDP elliptiques donnent des descriptions aussi précises dans les espaces $C^{k}$ ( $k$ dépendant du degré de régularité dans la convergence de la suite $\left(h_{\alpha}\right)$ ). Ainsi la figure 4 , page 34 , est parfaitement justifiée. La suite $\left(u_{\alpha}\right)$ ressemble réellement à la somme d'une solution régulière de l'équation limite et d'un certain nombre de bulles.

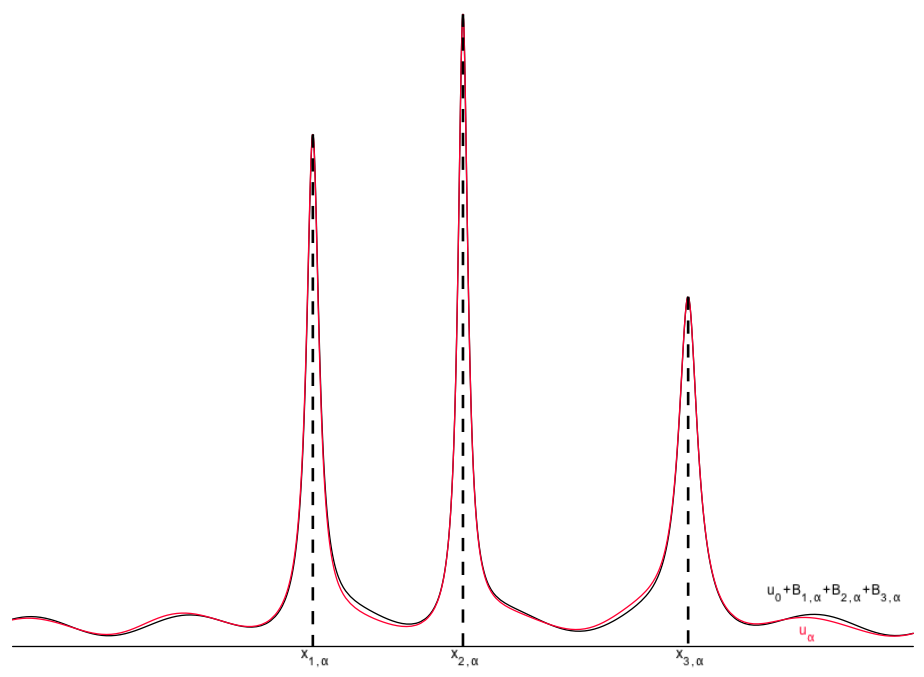

Fig. 4 : théorie ponctuelle sur les $\left(u_{\alpha}\right)$

Nous verrons dans la section 3.3 comment obtenir des informations précises sur les configurations de bulles possibles dans un tel processus. Si des bulles peuvent se former sur des bulles, ceci ne peut être fait de manière complètement arbitraire. 
Le reste de cette section est consacré à l'exposition des idées essentielles de la preuve du théorème $3.1^{2}$.

\subsubsection{Comment trouver les points de concentration?}

Même si les techniques de type "énergie", basées sur la fonction de concentration de Lévy (cf. [94]), permettent de détecter toutes les bulles qui peuvent se développer dans notre cas précis ${ }^{3}$, nous allons décrire comment détecter les points de concentration par une méthode ponctuelle qui nous mènera au résultat principal de cette sous-section, la proposition 3.1, page 44. L'énorme avantage de cette méthode est, outre sa souplesse qui lui permet de s'adapter à des équations où la méthode d'énergie est inopérante, qu'elle fournit automatiquement des estimées ponctuelles sur la suite $\left(u_{\alpha}\right)$ dépendant de la distance à laquelle se trouve l'ensemble des points de concentration. Son (léger) inconvénient est évidemment qu'elle demande un peu plus de régularité sur la suite $\left(h_{\alpha}\right)$. Cette méthode de détection repose fondamentalement sur l'invariance des termes dominants de l'équation, i.e. $\Delta_{g} u_{\alpha}$ et $u_{\alpha}^{\frac{n+2}{n-2}}$, par le changement d'échelle $u \mapsto \lambda^{\frac{n-2}{2}} u(\lambda x)$ Bien entendu, ce changement d'échelle n'a aucun sens global sur une variété mais, comme les phénomènes de concentration se déroulent à des échelles microscopiques et comme une variété riemannienne n'est infinitésimalement qu'un espace euclidien, il est possible de donner un sens à ce changement d'échelle sur les domaines qui vont nous intéresser. La méthode décrite ci-dessous se compose de deux étapes : premièrement, il faut trouver le changement d'échelle qui permette de voir quelque chose au voisinage d'un point de concentration; deuxièmement, il faut trouver une estimée ponctuelle ayant les mêmes invariances que l'équation - ou tout du moins que ces composantes non compactes - qui fournira, si elle est violée, un nouveau point de concentration. La première étape est également utilisée dans les méthodes d'énergie. Elle consiste à regarder à la loupe la solution $\left(u_{\alpha}\right) \mathrm{au}$ voisinage d'un point où elle se concentre - que ce soit l'énergie, i.e. la norme $H_{1}^{2}$ ici, qui se concentre, ou que ce soit une concentration ponctuelle, i.e. en norme $L^{\infty}$ - et à écraser celle-ci afin qu'elle ne se concentre plus. Il faut alors montrer que la suite, vue sous cet angle, converge vers un profil standard (ce qui demande un résultat de classification des solutions de l'équation-modèle associée). La deuxième étape est l'idée essentielle de la méthode ponctuelle. Ces deux étapes s'adaptent à beaucoup d'équations présentant des défauts de compacité dus à l'invariance de celles-ci par un certain changement d'échelle. Trouver l'estimée ponctuelle invariante qui permet de faire fonctionner la deuxième étape est souvent le point délicat. Nous reviendrons sur ces diverses difficultés (classification des solutions de

2. Il est tout-à-fait exclu de la présenter exhaustivement puisqu'elle court sur 120 pages et ce n'est pas l'objet de ce texte. Nous renvoyons à [42] .

3. C'est d'ailleurs ainsi que la décomposition $H_{1}^{2}$ ci-dessus est obtenue. 
l'équation-modèle associée, recherche du changement d'échelle adapté et de l'estimée ponctuelle adéquate) dans le chapitre 4 lorsque nous traiterons d'autres équations.

\section{Première étape : Détection du premier point de concentration}

Le candidat le plus naturel pour être point de concentration est le ou un - point où la fonction $u_{\alpha}$ est maximale. Remarquons tout d'abord que, si la suite $\left(u_{\alpha}\right)$ était uniformément bornée dans $L^{\infty}(M)$, les résultats de théorie elliptique standard donneraient alors gratuitement le résultat du théorème avec $N=0$. En d'autres termes, nous aurions, à extraction d'une sous-suite près, convergence dans l'espace $C^{2}(M)$ de la suite $\left(u_{\alpha}\right)$ vers une limite $u_{0}$ solution de l'équation-limite. Dans ce cas d'ailleurs, il est certain que cette limite est non-nulle (cf. [42] pour la preuve, simple, de cette assertion). A partir de maintenant, nous supposerons donc que

$$
\sup _{M} u_{\alpha} \rightarrow+\infty \text { quand } \alpha \rightarrow+\infty \text {. }
$$

Soit alors $\left(x_{\alpha}\right)$ une suite de points de $M$ telle que

$$
u_{\alpha}\left(x_{\alpha}\right)=\sup _{M} u_{\alpha}
$$

Regardons cette suite $\left(u_{\alpha}\right)$ au microscope au voisinage de $x_{\alpha}$ et écrasons-là afin d'y voir plus clair. Pour ce faire, on pose, pour $x \in B_{0}\left(\delta u_{\alpha}\left(x_{\alpha}\right)^{\frac{2}{n-2}}\right) \subset$ $\mathbb{R}^{n}, 0<\delta<\frac{1}{2} i_{g}(M), i_{g}(M)$ étant le rayon d'injectivité de $M$,

$$
\begin{aligned}
& v_{\alpha}(x)=u_{\alpha}\left(x_{\alpha}\right)^{-1} u_{\alpha}\left(\exp _{x_{\alpha}}\left(u_{\alpha}\left(x_{\alpha}\right)^{-\frac{2}{n-2}} x\right)\right) \text { et } \\
& g_{\alpha}(x)=\exp _{x_{\alpha}}^{\star} g\left(u_{\alpha}\left(x_{\alpha}\right)^{-\frac{2}{n-2}} x\right) .
\end{aligned}
$$

Passer dans la carte exponentielle permet de donner un sens au changement d'échelle effectué. Celui-ci est lié aux invariances de l'équation (cf. la discussion ci-dessus). En effet, la suite $\left(v_{\alpha}\right)$ vérifie l'équation

$$
\Delta_{g_{\alpha}} v_{\alpha}+u_{\alpha}\left(x_{\alpha}\right)^{-\frac{4}{n-2}} h_{\alpha}\left(\exp _{x_{\alpha}}\left(u_{\alpha}\left(x_{\alpha}\right)^{-\frac{2}{n-2}} \cdot\right)\right) v_{\alpha}=v_{\alpha}^{\frac{n+2}{n-2}}
$$

dans $B_{0}\left(\delta u_{\alpha}\left(x_{\alpha}\right)^{\frac{2}{n-2}}\right)$. Ce changement d'échelle laisse bien invariant les deux termes non-compacts de l'équation et rend négligeable le terme linéaire qui, lui, est compact. De plus, grâce à notre choix de $x_{\alpha}, 0 \leq v_{\alpha} \leq$ $v_{\alpha}(0)=1$ dans $B_{0}\left(\delta u_{\alpha}\left(x_{\alpha}\right)^{\frac{2}{n-2}}\right)$. Comme $u_{\alpha}\left(x_{\alpha}\right) \rightarrow+\infty$ quand $\alpha \rightarrow$ $+\infty, g_{\alpha} \rightarrow \xi$ dans $C_{l o c}^{2}\left(\mathbb{R}^{n}\right)$ quand $\alpha \rightarrow+\infty$ où $\xi$ est la métrique euclidienne. Par théorie elliptique standard, moyennant une borne $L^{\infty}$ sur $\left(h_{\alpha}\right)$ 
par exemple, il existe une sous-suite de $\left(v_{\alpha}\right)$ qui converge dans $C_{\text {loc }}^{1}\left(\mathbb{R}^{n}\right)$ vers une solution $U$ (régulière) de l'équation-modèle

$$
\Delta_{\xi} U=U^{\frac{n+2}{n-2}}
$$

dans $\mathbb{R}^{n}$. De plus, par passage à la limite, $0 \leq U \leq U(0)=1$. C'est à ce moment-là qu'est absolument nécessaire une classification de l'ensemble des solutions de l'équation-modèle. Ici, cette classification est particulièrement simple puisque toutes les solutions positives de l'équation ci-dessus sont de la forme

$$
U(x)=\lambda^{\frac{n-2}{2}}\left(1+\frac{\lambda^{2}\left|x-x_{0}\right|^{2}}{n(n-2)}\right)^{1-\frac{n}{2}}
$$

pour un certain $x_{0} \in \mathbb{R}^{n}$ et un certain $\lambda>0$. Cette classification est due à Caffarelli-Gidas-Spruck [22]. Dans notre cas, c'est-à-dire lorsque les solutions sont a priori dans $L^{\frac{2 n}{n-2}}\left(\mathbb{R}^{n}\right)$, elle est en fait due à Obata [79]. La preuve d'Obata est purement géométrique puisqu'il est possible lorsque l'énergie est finie (ce qui correspond moralement à une décroissance des solutions à l'infini ${ }^{4}$ ) de remonter les solutions sur la sphère et de se ramener au problème de la classification des métriques dans la classe conforme de la sphère standard qui sont à courbure scalaire constante (ce qui revient à caractériser le groupe des difféomorphismes conformes de la sphère standard). Il convient de noter que c'est la non-compacité de ce groupe de difféomorphismes conformes de la sphère standard qui est cause des phénomènes de perte de compacité dans des équations de type Yamabe mais que c'est grâce au fait que cette perte de compacité vienne de ce problème géométrique qu'on peut classifier les profils d'explosion (i.e. les solutions de l'équation-modèle). Une preuve du résultat d'Obata utilisant la technique des "moving-planes" due à Alexandrov [5] a ensuite été trouvée par Gidas-Ni-Nirenberg [47]. La classification générale de [22] est basée sur une adaptation de la méthode des "moving-planes" lorsque la fonction n'a a priori pas de décroissance à l'infini - l'étape cruciale de la preuve étant alors de démontrer qu'il y a suffisamment de directions avec décroissance pour pouvoir démarrer la méthode des "moving-planes". Une preuve plus simple - plus géométrique et plus élégante - de cette classification, basée sur une méthode des "moving-spheres" (qui n'utilise que le caractère conformément invariant de l'équation et un principe du maximum) a été donnée plus récemment par Li-Zhang [68]. Avec cette classification, puisque $U$ admet son maximum de valeur 1 en 0 , il est clair que

$$
U(x)=\left(1+\frac{|x|^{2}}{n(n-2)}\right)^{1-\frac{n}{2}} .
$$

4. Ceci peut d'ailleurs être rigoureusement démontré, cf. par exemple [41]. 
Nous avons donc bien obtenu un profil de $u_{\alpha}$ au voisinage de $x_{\alpha}$. En effet, en remontant ce résultat sur la variété, nous avons

$$
u_{\alpha}=(1+o(1)) B_{1, \alpha}
$$

dans toute boule $B_{x_{1, \alpha}}\left(R \mu_{1, \alpha}\right)$ où $x_{1, \alpha}=x_{\alpha}, \mu_{1, \alpha}=u_{\alpha}\left(x_{\alpha}\right)^{-\frac{2}{n-2}}$ et $B_{1, \alpha}$ est la bulle-standard de centre $x_{1, \alpha}$ et de poids $\mu_{1, \alpha}$ comme définie en (3.2).

Nous avons donc trouvé notre premier point de concentration. Ce procédé classique de changement d'échelle remonte à Struwe [94]. Mais la technique ponctuelle directe développée ici remonte plutôt à Schoen [89].

\section{Seconde étape : Détection des bulles "hautes"}

Nous allons expliquer maintenant comment détecter un premier paquet de bulles, dites bulles "hautes". La terminologie s'éclaircira lors de l'étape suivante quand nous aurons un deuxième paquet de bulles à comparer à ce premier. L'idée essentielle est de concocter une estimée ponctuelle invariante par le changement d'échelle utilisé dans la section précédente pour obtenir un profil asymptotique autour d'un point de concentration. Une telle estimée ponctuelle donnera souvent, si ce n'est toujours, un moyen de détecter d'autres points de concentration. Pour illustrer cette méthode, voyons comment elle permet de construire le deuxième point de concentration, les autres points de ce premier paquet s'obtenant de manière similaire. Posons

$$
\Phi_{\alpha}(x)=d_{g}\left(x_{1, \alpha}, x\right)^{\frac{n-2}{2}} u_{\alpha}(x) .
$$

Cette quantité est invariante par le changement d'échelle ci-dessus. En effet,

$$
\begin{aligned}
\Phi_{\alpha}\left(\exp _{x_{1, \alpha}}\left(\mu_{1, \alpha} x\right)\right) & =\mu_{1, \alpha}^{\frac{n-2}{2}}|x|^{\frac{n-2}{2}} u_{\alpha}\left(\exp _{x_{1, \alpha}}\left(\mu_{1, \alpha} x\right)\right) \\
& =|x|^{\frac{n-2}{2}} v_{\alpha}(x)
\end{aligned}
$$

ce qui n'est rien d'autre que la quantité ci-dessus construite cette fois à partir de la fonction $v_{\alpha}$.

Cette quantité permet de détecter un deuxième point de concentration de la façon suivante : supposons que $\sup _{M} \Phi_{\alpha} \rightarrow+\infty$ quand $\alpha \rightarrow+\infty$. Alors, en prenant $y_{\alpha}$ un point de maximum de $\Phi_{\alpha}$, il est possible de répéter l'étape de la sous-section précédente en remplaçant $x_{\alpha}$ par $y_{\alpha}$. En effet, ce choix de $y_{\alpha}$ assure que la suite de fonctions renormalisées est localement uniformément bornée, ce qui permet une nouvelle fois de passer à la limite dans l'équation et d'obtenir la convergence vers un profil standard. Le point essentiel ici est que l'hypothèse $\Phi_{\alpha}\left(y_{\alpha}\right) \rightarrow+\infty$ assure que, après le changement d'échelle approprié autour de $y_{\alpha}$, le premier point de 
concentration est bien envoyé à l'infini, ce qui se traduit par les relations suivantes :

$$
\frac{d_{g}\left(x_{1, \alpha}, y_{\alpha}\right)}{u_{\alpha}\left(x_{1, \alpha}\right)^{-\frac{2}{n-2}}+u_{\alpha}\left(y_{\alpha}\right)^{-\frac{2}{n-2}}} \rightarrow+\infty \text { quand } \alpha \rightarrow+\infty .
$$

En un sens, cette étape permet de détecter des bulles qui sont relativement éloignées les unes des autres (leur distance respective étant mesurée par rapport à leur poids).

Pour continuer le processus, il suffit de remplacer la quantité $\Phi_{\alpha}$ par

$$
\left(\min _{i=1, \ldots, k} d_{g}\left(x_{i, \alpha}, x\right)\right)^{\frac{n-2}{2}} u_{\alpha}(x)
$$

une fois les $k$ premiers points de concentration construits. Tant que cette quantité n'est pas uniformément bornée, son maximum donne un nouveau point de concentration. Le processus s'arrête nécessairement grâce à la borne a priori sur l'énergie que nous avons faite. En effet, un argument simple (déjà présent dans [94]) permet de montrer que les bulles ainsi construites n'interagissent pas au niveau $H_{1}^{2}$ et que leur propre énergie est quantifiée. Chaque bulle apporte un quantum d'énergie $E_{n}$ et $k$ bulles ainsi construites en apportent $k E_{n}$. La borne sur l'énergie borne donc a priori le nombre de points de concentration que ce processus peut produire. Pour une discussion des difficultés soulevées par l'absence de borne sur l'énergie, nous renvoyons à la section 3.5 .

Une fois que le processus s'arrête, nous avons non seulement un certain nombre de points de concentration $\left(x_{1, \alpha}, \ldots, x_{k, \alpha}\right)$ avec des poids associés $\left(\mu_{1, \alpha}, \ldots, \mu_{k, \alpha}\right)$ et un profil asymptotique de $u_{\alpha}$ au voisinage de ces points, i.e.

$$
u_{\alpha}=(1+o(1)) B_{i, \alpha}
$$

dans la boule $B_{x_{i, \alpha}}\left(R \mu_{i, \alpha}\right)$, mais également une estimée ponctuelle sur $u_{\alpha}$, i.e.

$$
\left(\min _{i=1, \ldots, k} d_{g}\left(x_{i, \alpha}, x\right)\right)^{\frac{n-2}{2}} u_{\alpha}(x) \leq C
$$

sur $M$ avec $C$ indépendant de $\alpha$.

Les bulles trouvées dans cette étape vérifient toutes

$$
\frac{d_{g}\left(x_{i, \alpha}, x_{j, \alpha}\right)}{\mu_{i, \alpha}} \rightarrow+\infty
$$

pour tous $i, j \in\{1, \ldots, k\}, i \neq j$.

L'estimée ponctuelle ci-dessus a déjà été utilisée de nombreuses fois dans le cas d'un point de concentration isolé. Elle remonte à Schoen-Zhang [93]. 


\section{Troisième étape : Détection des bulles "basses" et "cachées}

Nous n'avons malheureusement pas trouvé toutes les bulles avec le mode de détection de la sous-section précédente. En particulier, nous n'avons pas trouvé de bulles sur des bulles, c'est-à-dire des couples de bulles ne vérifiant pas la relation (3.5). En fait, il nous manque dans ces couples les plus basses (et donc les plus étalées), celles dont le maximum est caché par celui de la bulle-compagnon, cf. figure 5 ci-dessous, page 41 .

Comment faire pour détecter ces bulles? Il faut raffiner la méthode précédente en regardant cette fois la quantité

$\Psi_{\alpha}(x)=\left(\min _{i=1, \ldots, k}\left(d_{g}\left(x_{i, \alpha}, x\right)+\mu_{i, \alpha}\right)\right)^{\frac{n-2}{2}}\left|u_{\alpha}(x)-u_{0}(x)-\sum_{i=1}^{k} B_{i, \alpha}(x)\right|$

Tant que cette quantité ne tend pas uniformément vers 0 , il est possible de trouver un nouveau point de concentration ${ }^{5}$. Montrons-le sur le cas le plus simple. Supposons donc que les $k$ points de concentration trouvés sont ceux de l'étape précédente. Remarquons tout d'abord que $\Psi_{\alpha}$ tend uniformément vers 0 sur $\bigcup_{i=1}^{k} B_{x_{i, \alpha}}\left(R \mu_{i, \alpha}\right)$ mais également sur $M \backslash \bigcup_{i=1}^{k} B_{x_{i, \alpha}}\left(\frac{1}{R}\right)$ pour tout $R>0$. Ceci est une conséquence de la sous-section précédente. Pour le deuxième point, nous avons juste besoin de remarquer que la suite $\left(u_{\alpha}\right)$ est uniformément bornée sur cet ensemble grâce à l'estimée faible obtenue lors de la première étape et d'utiliser des résultats de théorie elliptique standard pour en déduire une convergence ponctuelle vers $u_{0}$ sur cet ensemble. Il nous reste donc à comprendre ce qui se passe dans la zone intermédiaire.

5. Remarque : cette quantité est toujours uniformément bornée une fois l'étape précédente achevée. 


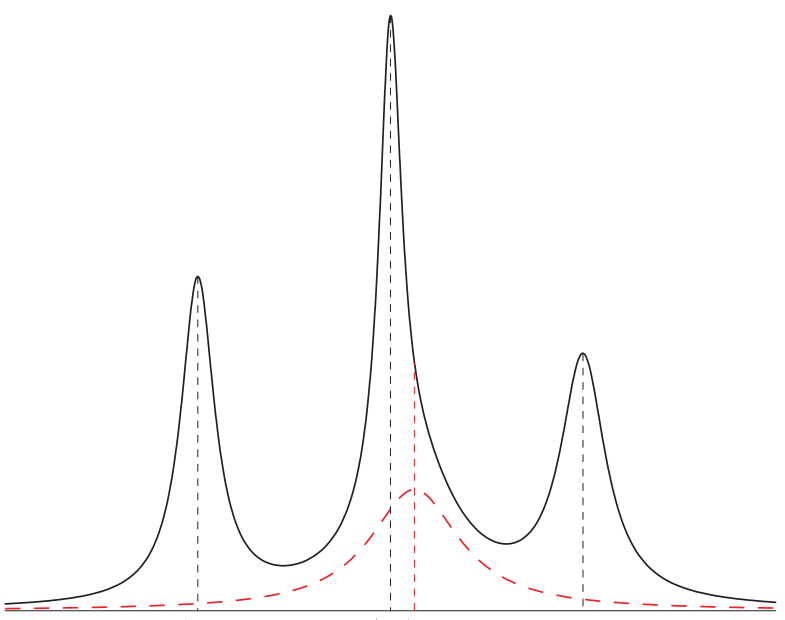

Fig. 5 : une bulle "basse" et "cachée" qu'il faut détecter.

Supposons que

$$
\Psi_{\alpha}\left(x_{\alpha}\right)=\sup _{M} \Psi_{\alpha} \geq \varepsilon_{0}
$$

pour un certain $\varepsilon_{0}>0$ indépendant de $\alpha$. Vu ce qui vient d'être remarqué, il est clair que

$$
\min _{i=1, \ldots, k} d_{g}\left(x_{i, \alpha}, x_{\alpha}\right) \rightarrow 0 \text { quand } \alpha \rightarrow+\infty
$$

et que

$$
\frac{d_{g}\left(x_{i, \alpha}, x_{\alpha}\right)}{\mu_{i, \alpha}} \rightarrow+\infty \text { quand } \alpha \rightarrow+\infty
$$

pour tout $1 \leq i \leq k$. Il est également clair que $u_{\alpha}\left(x_{\alpha}\right) \rightarrow+\infty$ quand $\alpha \rightarrow+\infty$. Posons

$$
\begin{aligned}
& v_{\alpha}(x)=u_{\alpha}\left(x_{\alpha}\right)^{-1} u_{\alpha}\left(\exp _{x_{\alpha}}\left(u_{\alpha}\left(x_{\alpha}\right)^{-\frac{2}{n-2}} x\right)\right) \text { et } \\
& g_{\alpha}(x)=\exp _{x_{\alpha}}^{\star} g\left(u_{\alpha}\left(x_{\alpha}\right)^{-\frac{2}{n-2}} x\right) .
\end{aligned}
$$

Pour tout $1 \leq i \leq k$, notons $\hat{x}_{i, \alpha}$ l'image du point de concentration $x_{i, \alpha}$ par ce changement d'échelle, i.e.

$$
\hat{x}_{i, \alpha}=u_{\alpha}\left(x_{\alpha}\right)^{\frac{2}{n-2}} \exp _{x_{\alpha}}^{-1}\left(x_{i, \alpha}\right)
$$


pour les points pour lesquels cela a un sens, c'est-à-dire pour ceux qui vérifient $d_{g}\left(x_{i, \alpha}, x_{\alpha}\right)<i_{g}(M)$. Mais peu importe car ceux qui nous intéressent sont ceux pour lesquels

$$
d_{g}\left(x_{i, \alpha}, x_{\alpha}\right)^{\frac{n-2}{2}} u_{\alpha}\left(x_{\alpha}\right)=O(1)
$$

qui vont donner, quitte à extraire une sous-suite, une trace dans $\mathbb{R}^{n}$, c'està-dire une limite de $\hat{x}_{i, \alpha}$ que nous noterons $\hat{x}_{i}$. Nous noterons également $\mathcal{I} \subset\{1, \ldots, k\}$ l'ensemble des $i$ pour lesquels cette limite existe et est finie. Il convient de remarquer que $\mathcal{I} \neq \emptyset$ grâce à l'estimée obtenue à la fin de la première étape d'exhaustion des points de concentration. Ceci illustre le fait que la nouvelle bulle que nous sommes en train de construire est "sous" celles correspondant aux points de concentration qui laissent une trace $\hat{x}_{i}$ après le changement d'échelle.

En utilisant l'estimée de la première étape, i.e.

$$
\left(\min _{i=1, \ldots, k} d_{g}\left(x_{i, \alpha}, x\right)\right)^{\frac{n-2}{2}} u_{\alpha}(x) \leq C,
$$

et puisque cette estimée est invariante par le changement d'échelle effectué, il est clair que

$$
\limsup _{\alpha \rightarrow+\infty} v_{\alpha}\left(z_{\alpha}\right) \leq C\left(\min _{i \in \mathcal{I}}\left|z-\hat{x}_{i}\right|\right)^{1-\frac{n}{2}}
$$

pour toute suite de points $\left(z_{\alpha}\right)$ convergeant vers $z \in \mathbb{R}^{n} \backslash\left\{\hat{x}_{i}\right\}_{i \in \mathcal{I}}$. En particulier, la suite $\left(v_{\alpha}\right)$ est uniformément bornée sur tout compact de $\mathbb{R}^{n} \backslash\left\{\hat{x}_{i}\right\}_{i \in \mathcal{I}}$ et, par théorie elliptique standard, après extraction d'une sous-suite, $v_{\alpha} \rightarrow V$ dans $C_{\text {loc }}^{1}\left(\mathbb{R}^{n} \backslash\left\{\hat{x}_{i}\right\}_{i \in \mathcal{I}}\right)$ quand $\alpha \rightarrow+\infty$ où $V$ vérifie

$$
\Delta_{\xi} V=V^{\frac{n+2}{n-2}} \text { dans } \mathbb{R}^{n} \backslash\left\{\hat{x}_{i}\right\}_{i \in \mathcal{I}}
$$

et

$$
0 \leq V \leq C\left(\min _{i \in \mathcal{I}}\left|z-\hat{x}_{i}\right|\right)^{1-\frac{n}{2}} .
$$

Grâce à cette estimée ponctuelle, les singularités de $V$ en $\hat{x}_{i}$ sont effaçables et $V \in C^{\infty}\left(\mathbb{R}^{n}\right)$ vérifie l'équation-limite sur tout l'espace ${ }^{6}$. Il reste à montrer que $V$ n'est pas identiquement nulle pour obtenir encore une fois

6. Ceci est également un point crucial. La classification des solutions de l'équationlimite avec singularités éventuelles peut poser un problème, même avec l'estimée ponctuelle correspondant à l'hypothèse d'énergie bornée. Voir par exemple section 4.6. Si des solutions singulières pouvaient exister, cela signifierait qu'une bulle haute pourrait "tordre" une bulle basse et éventuellement lui faire perdre de l'énergie. 
un profil standard. Mais ceci est dû au fait que $\Psi_{\alpha}\left(x_{\alpha}\right) \geq \varepsilon_{0}>0$. En remarquant grâce à (3.6) et (3.7) que

$$
\left(d_{g}\left(x_{i, \alpha}, x_{\alpha}\right)+\mu_{i, \alpha}\right)^{\frac{n-2}{2}} B_{i, \alpha}\left(x_{\alpha}\right) \rightarrow 0 \text { quand } \alpha \rightarrow+\infty
$$

et que

$$
\left(\min _{i=1, \ldots, k}\left(d_{g}\left(x_{i, \alpha}, x_{\alpha}\right)+\mu_{i, \alpha}\right)\right)^{\frac{n-2}{2}} u_{0}\left(x_{\alpha}\right) \rightarrow 0 \text { quand } \alpha \rightarrow+\infty,
$$

nous obtenons directement $\left|\hat{x}_{i}\right| \geq \varepsilon_{0}^{\frac{2}{n-2}}$ pour tout $i \in \mathcal{I}$. La convergence de $v_{\alpha}$ vers $V$ ayant ainsi lieu dans $C^{1}$ dans un petit voisinage de 0 et $v_{\alpha}(0)$ étant égal à 1 , il est clair que $V(0)=1$. Le résultat de classification de [22] nous donne alors l'existence de $x_{0} \in \mathbb{R}^{n}$ et de $\lambda>0$ tels que

$$
V(x)=\lambda^{\frac{n-2}{2}}\left(\lambda^{2}+\frac{\left|x-x_{0}\right|^{2}}{n(n-2)}\right)^{1-\frac{n}{2}} .
$$

En posant $x_{k+1, \alpha}=\exp _{x_{\alpha}}\left(u_{\alpha}\left(x_{\alpha}\right)^{-\frac{2}{n-2}} x_{0}\right)$ et $\mu_{k+1, \alpha}=\left(\lambda u_{\alpha}\left(x_{\alpha}\right)\right)^{-\frac{2}{n-2}}$, nous sommes arrivés à

$$
\mu_{k+1, \alpha}^{\frac{n-2}{2}} u_{\alpha}\left(\exp _{x_{k+1, \alpha}}\left(\mu_{k+1, \alpha} x\right)\right) \rightarrow U(x)=\left(1+\frac{|x|^{2}}{n(n-2)}\right)^{1-\frac{n}{2}}
$$

dans $C_{\text {loc }}^{2}\left(\mathbb{R}^{n} \backslash \mathcal{S}_{k+1}\right)$ quand $\alpha \rightarrow+\infty$ où

$$
\begin{array}{r}
\mathcal{S}_{k+1}=\left\{\lim _{\alpha \rightarrow+\infty} \frac{1}{\mu_{k+1, \alpha}} \exp _{x_{k+1, \alpha}}^{-1}\left(x_{i, \alpha}\right), 1 \leq i \leq k\right. \text { t.q. } \\
\left.d_{g}\left(x_{i, \alpha}, x_{k+1, \alpha}\right)=O\left(\mu_{k+1, \alpha}\right)\right\}
\end{array}
$$

Il est clair que la bulle ainsi trouvée correspond bien à celle de la figure 5 , page 41. En particulier, il existe nécessairement au moins une bulle qui a son centre dans la boule de centre $x_{k+1, \alpha}$ et de rayon $R \mu_{k+1, \alpha}$ pour $R$ assez grand, c'est-à-dire une bulle qui est vraiment sur elle.

De même que dans l'étape précédente, toute nouvelle bulle ainsi construite n'interagit pas, au niveau $H_{1}^{2}$, avec les précédentes, et apporte avec elle un quantum d'énergie. Quitte à répéter le processus ${ }^{7}$, processus qui s'arrêtera nécessairement grâce à la borne a priori sur l'énergie des solutions, nous arrivons au résultat suivant :

7. Le cas général, i.e. lorsqu'on a déjà des bulles de second type, est techniquement plus compliqué mais fondamentalement similaire à celui que nous venons de traiter. 
Proposition 3.1. Soit $(M, g)$ une variété riemannienne compacte de dimension $n \geq 3$. Soit $\left(u_{\alpha}\right)$ une suite de solutions $C^{2}$ positives de l'équation

$$
\Delta_{g} u_{\alpha}+h_{\alpha} u_{\alpha}=u_{\alpha}^{\frac{n+2}{n-2}}
$$

avec $h_{\alpha} \rightarrow h_{0}$ dans $C^{0, \eta}(M)$ pour un certain $\eta>0$. Alors il existe $u_{0} \in$ $C^{2}(M)$ solution de

$$
\Delta_{g} u_{0}+h_{0} u_{0}=u_{0}^{\frac{n+2}{n-2}}
$$

qui est soit identiquement nulle, soit strictement positive, $N \in \mathbb{N}$ et $N$ suites $\left(x_{i, \alpha}, \mu_{i, \alpha}\right)_{i=1, \ldots, N}$ où $x_{i, \alpha} \in M$ et $\mu_{i, \alpha}>0$ vérifie $\mu_{i, \alpha} \rightarrow 0$ quand $\alpha \rightarrow+\infty$ telles que les propriétés suivantes aient lieu après extraction d'une sous-suite :

(a) $u_{\alpha} \rightarrow u_{0}$ dans $C_{\text {loc }}^{2}(M \backslash \mathcal{S})$ quand $\alpha \rightarrow+\infty$ où $\mathcal{S}=\left\{\lim _{\alpha \rightarrow+\infty} x_{i, \alpha}\right\}_{i=1, \ldots, N}$.

(b) Pour tout $1 \leq i \leq N$,

$$
\mu_{i, \alpha}^{\frac{n-2}{2}} u_{\alpha}\left(\exp _{x_{i, \alpha}}\left(\mu_{i, \alpha} x\right)\right) \rightarrow\left(1+\frac{|x|^{2}}{n(n-2)}\right)^{1-\frac{n}{2}}
$$

dans $C_{\text {loc }}^{2}\left(M \backslash \mathcal{S}_{i}\right)$ quand $\alpha \rightarrow+\infty$ où

$$
\mathcal{S}_{i}=\left\{\lim _{\alpha \rightarrow+\infty} \frac{1}{\mu_{i, \alpha}} \exp _{x_{i, \alpha}}\left(x_{j, \alpha}\right), j \neq i \text { t.q. } d_{g}\left(x_{i, \alpha}, x_{j, \alpha}\right)=O\left(\mu_{i, \alpha}\right)\right\} .
$$

(c) Pour tous $i, j \in\{1, \ldots, N\}, i \neq j$,

$$
\frac{d_{g}\left(x_{i, \alpha}, x_{j, \alpha}\right)^{2}}{\mu_{i, \alpha} \mu_{j, \alpha}}+\frac{\mu_{i, \alpha}}{\mu_{j, \alpha}}+\frac{\mu_{j, \alpha}}{\mu_{i, \alpha}} \rightarrow+\infty \text { quand } \alpha \rightarrow+\infty .
$$

(d) La quantité

$$
\left(\min _{i=1, \ldots, N}\left(d_{g}\left(x_{i, \alpha}, x\right)+\mu_{i, \alpha}\right)\right)^{\frac{n-2}{2}}\left|u_{\alpha}(x)-u_{0}(x)-\sum_{i=1}^{N} B_{i, \alpha}(x)\right|
$$

tend vers 0 dans $L^{\infty}(M)$ quand $\alpha \rightarrow+\infty$ où

$$
B_{i, \alpha}(x)=\mu_{i, \alpha}^{\frac{n-2}{2}}\left(\mu_{i, \alpha}^{2}+\frac{d_{g}\left(x_{i, \alpha}, x\right)^{2}}{n(n-2)}\right)^{1-\frac{n}{2}} .
$$

En fait, les points (a) et (b) sont des conséquences de (c) et (d) avec un peu de théorie elliptique standard. Le point (c) est bien connu et n'est que l'indépendance des bulles dans l'espace d'énergie $H_{1}^{2}$. Une fois que ces trois 
étapes ont été menées à bien et que nous avons démontré la proposition 3.1 , nous avons en fait trouvé tous les points de concentration. Ceci n'est pas encore démontré et l'estimée (d) de la proposition 3.1 ne suffit pas pour l'obtenir. Elle est en effet critique pour les espaces d'énergie ${ }^{8}$ et ne permet donc pas de montrer qu'il n'y a pas d'énergie perdue entre les bulles et pas d'autres points de concentration. Mais cette estimée ponctuelle (faible) peut être améliorée en une estimée ponctuelle optimale. Il va nous falloir plusieurs étapes pour y arriver. C'est l'objet des sous-sections suivantes.

\subsubsection{Un premier contrôle ponctuel de la suite}

L'objectif est d'obtenir un contrôle par au-dessus de la suite $\left(u_{\alpha}\right)$ par la somme des bulles et de $u_{0}$, ce qui sera achevé dans la sous-section suivante. Pour l'instant, nous nous proposons d'esquisser la preuve du résultat suivant :

Proposition 3.2. Soit $(M, g)$ une variété riemannienne compacte de dimension $n \geq 3$. Soit $\left(u_{\alpha}\right)$ une suite de solutions $C^{2}$ positives de l'équation

$$
\Delta_{g} u_{\alpha}+h_{\alpha} u_{\alpha}=u_{\alpha}^{\frac{n+2}{n-2}}
$$

avec $h_{\alpha} \rightarrow h_{0}$ dans $C^{0, \eta}(M)$ pour un certain $\eta>0$. Alors il existe $C>0$ telle que, après extraction d'une sous-suite,

$$
\left|u_{\alpha}(x)-u_{0}(x)\right| \leq C \mu_{\alpha}^{\frac{n-2}{2}}\left(\min _{i=1, \ldots, N}\left(d_{g}\left(x_{i, \alpha}, x\right)+\mu_{i, \alpha}\right)\right)^{2-n}+\varepsilon_{\alpha}\left\|u_{0}\right\|_{\infty}
$$

pour tout $x \in M$ où les suites $\left(x_{i, \alpha}\right)_{i=1, \ldots, N}$ et $\left(\mu_{i, \alpha}\right)_{i=1, \ldots, N}$ et la fonction $u_{0}$ sont données par la proposition 3.1, $\mu_{\alpha}=\max _{i=1, \ldots, N} \mu_{i, \alpha}$, et où $\left(\varepsilon_{\alpha}\right)$ est une suite de réels strictement positifs tendant vers 0 quand $\alpha \rightarrow+\infty$.

Nous allons nous contenter d'esquisser la preuve de cette proposition dans le cas, beaucoup plus simple techniquement, où $u_{0} \equiv 0$ et nous renvoyons à [42] pour le cas général. La preuve se décompose en deux étapes, la première donnant une estimée intermédiaire entre celle de la proposition 3.1 (point $(\mathrm{d})$ ) et celle voulue ici.

Etape 1 (estimée intermédiaire) - Pour tout $0<\varepsilon<\frac{1}{2}$, il existe $R_{\varepsilon}>0$ et $C_{\varepsilon}>0$ tels que

$$
u_{\alpha}(x) \leq C_{\varepsilon} \mu_{\alpha}^{\frac{n-2}{2}(1-2 \varepsilon)} r_{\alpha}(x)^{(2-n)(1-\varepsilon)}
$$

8. L'estimée de décroissance en distance aux points de concentration à la puissance $1-\frac{n}{2}$ ne permet tout juste pas d'appliquer le théorème de convergence dominée de Lebesgue pour contrôler la norme $L^{\frac{2 n}{n-2}}$. Il est tout-à-fait normal que l'estimée ponctuelle invariante par le changement d'échelle qui laisse invariant l'équation soit également l'estimée ponctuelle qui constitue la limite pour un théorème de convergence dominée dans l'intégrale d'énergie. 
pour tout $x \in M \backslash \bigcup_{i=1}^{N} B_{x_{i, \alpha}}\left(R_{\varepsilon} \mu_{i, \alpha}\right)$ où

$$
r_{\alpha}(x)=\min _{i=1, \ldots, N} d_{g}\left(x_{i, \alpha}, x\right)
$$

La preuve de cette estimée repose sur deux ingrédients, l'estimée ponctuelle de la proposition 3.1 et le principe du maximum, combinés avec un bon choix de fonctions-tests. Il suffit en fait de montrer cette estimée pour des $\varepsilon$ suffisamment petits puisqu'elle s'améliore quand $\varepsilon$ décroît. L'opérateur $\Delta_{g}+h_{0}$ étant supposé sans noyau, il est coercif. Ainsi il existe $\varepsilon_{0}>0$ tel que l'opérateur $\Delta_{g}+h_{0}-\varepsilon_{0}$ soit également coercif. Soit $G$ la fonction de Green de cet opérateur, i.e. la fonction symétrique $G: M \times M \backslash\{(x, x), x \in M\} \mapsto \mathbb{R}$ vérifiant

$$
\Delta_{g, y} G(x, y)+\left(h_{0}(y)-\varepsilon_{0}\right) G(x, y)=\delta_{x}
$$

au sens des distributions pour tout $x \in M$. Cette fonction de Green est strictement positive et, cf. [42], il existe $C_{1}>0, C_{2}>0$ et $C_{3}>0$ telles que

$$
\frac{1}{C_{1}} \leq d_{g}(x, y)^{n-2} G(x, y) \leq C_{1}
$$

et

$$
\frac{|\nabla G(x, y)|^{2}}{G(x, y)^{2}} \geq C_{2} d_{g}(x, y)^{-2}-C_{3}
$$

pour tous $(x, y) \in M^{2}, x \neq y$. Posons

$$
\Phi_{\alpha, \varepsilon}(x)=\sum_{i=1}^{N} G\left(x_{i, \alpha}, x\right)^{1-\varepsilon}
$$

pour $x \in M \backslash\left\{x_{i, \alpha}\right\}_{i=1, \ldots, N}$. Soit maintenant un point $x_{\alpha} \in M$ maximum local de $\frac{u_{\alpha}}{\Phi_{\alpha, \varepsilon}}$. Comme $\nabla\left(\frac{u_{\alpha}}{\Phi_{\alpha, \varepsilon}}\right)\left(x_{\alpha}\right)=0$ et $\Delta_{g}\left(\frac{u_{\alpha}}{\Phi_{\alpha, \varepsilon}}\right)\left(x_{\alpha}\right) \geq 0$,

$$
\frac{\Delta_{g} u_{\alpha}\left(x_{\alpha}\right)}{u_{\alpha}\left(x_{\alpha}\right)} \geq \frac{\Delta_{g} \Phi_{\alpha, \varepsilon}\left(x_{\alpha}\right)}{\Phi_{\alpha, \varepsilon}\left(x_{\alpha}\right)} .
$$

Ceci donne

$$
\frac{\Delta_{g} \Phi_{\alpha, \varepsilon}\left(x_{\alpha}\right)}{\Phi_{\alpha, \varepsilon}\left(x_{\alpha}\right)} \leq u_{\alpha}\left(x_{\alpha}\right)^{\frac{4}{n-2}}-h_{\alpha}\left(x_{\alpha}\right) .
$$


Il reste à remarquer par un simple calcul que

$$
\begin{aligned}
\Delta_{g} \Phi_{\alpha, \varepsilon}\left(x_{\alpha}\right)= & -\left(h_{0}\left(x_{\alpha}\right)-\varepsilon_{0}\right)(1-\varepsilon) \sum_{i=1}^{N} G\left(x_{i, \alpha}, x_{\alpha}\right)^{1-\varepsilon} \\
& +\varepsilon(1-\varepsilon) \sum_{i=1}^{N} \frac{\left|\nabla G\left(x_{i, \alpha}, x_{\alpha}\right)\right|^{2}}{G\left(x_{i, \alpha}, x_{\alpha}\right)^{2}} G\left(x_{i, \alpha}, x_{\alpha}\right)^{1-\varepsilon} \\
= & -\left(h_{0}\left(x_{\alpha}\right)-\varepsilon_{0}\right)(1-\varepsilon) \Phi_{\alpha, \varepsilon} \\
& +\varepsilon(1-\varepsilon) \sum_{i=1}^{N} \frac{\left|\nabla G\left(x_{i, \alpha}, x_{\alpha}\right)\right|^{2}}{G\left(x_{i, \alpha}, x_{\alpha}\right)^{2}} G\left(x_{i, \alpha}, x_{\alpha}\right)^{1-\varepsilon}
\end{aligned}
$$

pour en déduire que

$$
\begin{aligned}
& \varepsilon(1-\varepsilon) \Phi_{\alpha, \varepsilon}\left(x_{\alpha}\right)^{-1} \sum_{i=1}^{N} \frac{\left|\nabla G\left(x_{i, \alpha}, x_{\alpha}\right)\right|^{2}}{G\left(x_{i, \alpha}, x_{\alpha}\right)^{2}} G\left(x_{i, \alpha}, x_{\alpha}\right)^{1-\varepsilon} \\
& \quad \leq u_{\alpha}\left(x_{\alpha}\right)^{\frac{4}{n-2}}-h_{\alpha}\left(x_{\alpha}\right)+\left(h_{0}\left(x_{\alpha}\right)-\varepsilon_{0}\right)(1-\varepsilon) \\
& \quad \leq u_{\alpha}\left(x_{\alpha}\right)^{\frac{4}{n-2}}-\varepsilon_{0}(1-\varepsilon)-\varepsilon h_{0}\left(x_{\alpha}\right)+o(1) \\
& \quad \leq u_{\alpha}\left(x_{\alpha}\right)^{\frac{4}{n-2}}-\frac{\varepsilon_{0}}{2}+o(1)
\end{aligned}
$$

quitte à choisir $\varepsilon$ suffisamment petit. En utilisant le point (a) de la proposition 3.1 et puisque $u_{0}$ est supposée nulle, on voit déjà, en utilisant que le terme de gauche est positif ou nul, que $r_{\alpha}\left(x_{\alpha}\right) \rightarrow 0$ quand $\alpha \rightarrow+\infty$. Il n'est alors pas difficile en utilisant (3.8) et (3.9) de minorer le terme de gauche par

$$
\varepsilon(1-\varepsilon) \frac{N}{C_{1}^{2-2 \varepsilon}}\left(C_{2} r_{\alpha}\left(x_{\alpha}\right)^{-2}-C_{3}\right)
$$

et d'en déduire que

$$
r_{\alpha}\left(x_{\alpha}\right)^{2} u_{\alpha}\left(x_{\alpha}\right)^{\frac{4}{n-2}} \geq \varepsilon(1-\varepsilon) \frac{N C_{2}}{C_{1}^{2-2 \varepsilon}}+o(1) .
$$

Grâce à l'estimée (d) de la proposition 3.1, il en découle que

$$
r_{\alpha}\left(x_{\alpha}\right)^{2}\left(\sum_{i=1}^{N} B_{i, \alpha}\left(x_{\alpha}\right)\right)^{\frac{4}{n-2}} \geq \varepsilon(1-\varepsilon) \frac{N C_{2}}{C_{1}^{2-2 \varepsilon}}+o(1) .
$$

Ceci n'est possible que si $x_{\alpha} \in \bigcup_{i=1}^{N} B_{x_{i, \alpha}}\left(R_{\varepsilon} \mu_{i, \alpha}\right)$ pour un certain $R_{\varepsilon}>0$ ne dépendant que de $\varepsilon$. Ainsi, la fonction $\frac{u_{\alpha}}{\Phi_{\alpha, \varepsilon}}$ n'admet pas de maxima 
locaux en-dehors de $\bigcup_{i=1}^{N} B_{x_{i, \alpha}}\left(R_{\varepsilon} \mu_{i, \alpha}\right)$. Ainsi

$$
\sup _{M \backslash \bigcup_{i=1}^{N} B_{x_{i, \alpha}}\left(R_{\varepsilon} \mu_{i, \alpha}\right)} \frac{u_{\alpha}}{\Phi_{\alpha}^{\varepsilon}}=\sup _{\partial\left(\bigcup_{i=1}^{N} B_{x_{i, \alpha}}\left(R_{\varepsilon} \mu_{i, \alpha}\right)\right)} \frac{u_{\alpha}}{\Phi_{\alpha}^{\varepsilon}} .
$$

Les estimées (3.8) et (3.9) et le point (b) de la proposition 3.1 permettent ensuite de conclure la preuve de cette première étape.

Etape 2 - Nous pouvons maintenant démontrer l'estimée de la proposition 3.2 qui correspond en gros à prendre $\varepsilon=0$ dans l'estimée de l'étape 1. Mais il y a un saut qualitatif entre $\varepsilon>0$ et $\varepsilon=0$. L'hypothèse $\varepsilon>0$ était cruciale dans l'étape précédente et il n'existe d'ailleurs pas à notre connaissance de preuve directe par principe du maximum de l'estimée finale (dans un cadre aussi général). Ici, nous allons utiliser la formule de représentation de Green pour obtenir le résultat. On note $G_{\alpha}$ la fonction de Green de l'opérateur $\Delta_{g}+h_{\alpha}$. Comme $h_{\alpha} \rightarrow h_{0}$ dans $C^{0, \eta}(M)$ quand $\alpha \rightarrow+\infty$, les estimées sur les fonctions de Green sont uniformes en $\alpha$ et nous avons en particulier l'estimée (3.8) sur $G_{\alpha}$ pour tout $\alpha$ assez grand ${ }^{9}$. Grâce à la formule de représentation de Green et à l'équation satisfaite par $u_{\alpha}$, nous pouvons ensuite écrire que

$u_{\alpha}\left(x_{\alpha}\right)=\int_{M} G_{\alpha}\left(x_{\alpha}, x\right) u_{\alpha}(x)^{\frac{n+2}{n-2}} d v_{g} \leq C_{1} \int_{M} d_{g}\left(x_{\alpha}, x\right)^{2-n} u_{\alpha}(x)^{\frac{n+2}{n-2}} d v_{g}$

pour toute suite $\left(x_{\alpha}\right)$ de points de $M$. Fixons $0<\varepsilon<\frac{2}{n+2}$ et utilisons l'estimée de l'étape 1 pour estimer une partie de cette intégrale. Plus précisément, écrivons que

$$
\begin{aligned}
& \int_{M \backslash \bigcup_{i=1}^{N} B_{x_{i, \alpha}}\left(R_{\varepsilon} \mu_{\alpha}\right)} d_{g}\left(x_{\alpha}, x\right)^{2-n} u_{\alpha}(x)^{\frac{n+2}{n-2}} d v_{g} \\
& \leq C_{\varepsilon}^{\frac{n+2}{n-2}} \mu_{\alpha}^{\frac{n+2}{2}(1-2 \varepsilon)} \int_{M \backslash \bigcup_{i=1}^{N} B_{x_{i, \alpha}}\left(R_{\varepsilon} \mu_{\alpha}\right)} d_{g}\left(x_{\alpha}, x\right)^{2-n} r_{\alpha}(x)^{-(n+2)(1-\varepsilon)} d v_{g} \\
& \leq C_{\varepsilon}^{\frac{n+2}{n-2}} \mu_{\alpha}^{\frac{n+2}{2}(1-2 \varepsilon)} \sum_{i=1}^{N} \int_{M \backslash B_{x_{i, \alpha}}\left(R_{\varepsilon} \mu_{\alpha}\right)} d_{g}\left(x_{\alpha}, x\right)^{2-n} d_{g}\left(x_{i, \alpha}, x\right)^{-(n+2)(1-\varepsilon)} d v_{g} \\
& =O\left(\sum_{i=1}^{N} \mu_{\alpha}^{\frac{n-2}{2}}\left(d_{g}\left(x_{i, \alpha}, x_{\alpha}\right)+\mu_{\alpha}\right)^{2-n}\right)
\end{aligned}
$$

par simple calcul puisque $\varepsilon<\frac{2}{n+2}$. Pour l'autre partie de l'intégrale, nous

9. Evidemment, la constante $C_{1}$ se doit d'être changée mais peut être choisie uniforme en $\alpha$. 
pouvons écrire que

$$
\begin{aligned}
& \int_{\bigcup_{i=1}^{N} B_{x_{i, \alpha}}\left(R_{\varepsilon} \mu_{\alpha}\right)} d_{g}\left(x_{\alpha}, x\right)^{2-n} u_{\alpha}(x)^{\frac{n+2}{n-2}} d v_{g} \\
& \quad=O\left(\sum_{i=1}^{N} d_{g}\left(x_{i, \alpha}, x_{\alpha}\right)^{2-n} \int_{B_{x_{i, \alpha}}\left(R_{\varepsilon} \mu_{\alpha}\right)} d_{g}\left(x_{\alpha}, x\right)^{2-n} u_{\alpha}(x)^{\frac{n+2}{n-2}} d v_{g}\right) \\
& =O\left(\sum_{i=1}^{N} \mu_{\alpha}^{\frac{n-2}{2}} d_{g}\left(x_{\alpha}, x_{i, \alpha}\right)^{2-n}\right)
\end{aligned}
$$

grâce aux inégalités de Hölder et à la borne a priori sur l'énergie dès lors que

$$
\frac{d_{g}\left(x_{i, \alpha}, x_{\alpha}\right)}{\mu_{\alpha}} \rightarrow+\infty \text { quand } \alpha \rightarrow+\infty
$$

pour tout $1 \leq i \leq N$. En combinant ces deux estimées, nous arrivons à

$$
u_{\alpha}\left(x_{\alpha}\right)=O\left(\mu_{\alpha}^{\frac{n-2}{2}}\left(\min _{i=1, \ldots, N}\left(d_{g}\left(x_{i, \alpha}, x\right)+\mu_{i, \alpha}\right)\right)^{2-n}\right)
$$

dès que la relation ci-dessus est vérifiée. Supposons maintenant que $r_{\alpha}\left(x_{\alpha}\right)$ $=O\left(\mu_{\alpha}\right)$. Le point $(\mathrm{d})$ de la proposition 3.1 donne alors immédiatement que

$$
\begin{aligned}
u_{\alpha}\left(x_{\alpha}\right)= & O\left(\sum_{i=1}^{N} \mu_{i, \alpha}^{\frac{n-2}{2}}\left(d_{g}\left(x_{i, \alpha}, x_{\alpha}\right)+\mu_{i, \alpha}\right)^{2-n}\right) \\
& +o\left(\left(\min _{i=1, \ldots, N}\left(d_{g}\left(x_{i, \alpha}, x_{\alpha}\right)+\mu_{i, \alpha}\right)\right)^{1-\frac{n}{2}}\right) \\
= & O\left(\mu_{\alpha}^{\frac{n-2}{2}}\left(\min _{i=1, \ldots, N}\left(d_{g}\left(x_{i, \alpha}, x_{\alpha}\right)+\mu_{i, \alpha}\right)\right)^{2-n}\right) \\
& +o\left(\left(\min _{i=1, \ldots, N}\left(d_{g}\left(x_{i, \alpha}, x_{\alpha}\right)+\mu_{i, \alpha}\right)\right)^{1-\frac{n}{2}}\right)
\end{aligned}
$$

ce qui donne le résultat voulu puisque

$$
\min _{i=1, \ldots, N}\left(d_{g}\left(x_{i, \alpha}, x_{\alpha}\right)+\mu_{i, \alpha}\right)=O\left(\mu_{\alpha}^{\frac{n-2}{2}}\right)
$$

dans ce cas. Ceci achève (l'esquisse de) la preuve de l'estimée de la proposition 3.2 .

Dans le cas où la limite faible $u_{0}$ est non-nulle, la preuve est essentiellement plus technique. Il faut tout d'abord démontrer une version locale de 
l'estimée à $\varepsilon$ près (c'est-à-dire au voisinage des points de concentration) pour l'étendre ensuite par des arguments de théorie elliptique standard à toute la variété. L'estimée obtenue n'est pas tout-à-fait la bonne, elle fait intervenir des termes supplémentaires légèrement singuliers (de moins en moins lorsque $\varepsilon \rightarrow 0$ ). De même, pour passer à l'estimée avec $\varepsilon=0$, il faut travailler un peu plus pour prendre soin de ces nouveaux termes. Mais le schéma général de la preuve reste dans l'ensemble le même.

Des estimées ponctuelles fortes de ce type, dans le cas d'un point de concentration isolé, i.e. dans le cas d'une bulle, remontent dans le cas euclidien à Han [51] et dans le cas vraiment riemannien (i.e. non localement conformément plat) à Hebey-Vaugon [56, 57].

\subsubsection{Hiérarchie des points de concentration et contrôle optimal}

L'objectif de cette étape est de parvenir à une estimée ponctuelle par audessus de la suite $\left(u_{\alpha}\right)$ qui soit optimale, c'est-à-dire un contrôle ponctuel de la suite $\left(u_{\alpha}\right)$ par la somme des bulles :

Proposition 3.3. Soit $(M, g)$ une variété riemannienne compacte de dimension $n \geq 3$. Soit $\left(u_{\alpha}\right)$ une suite de solutions $C^{2}$ positives de l'équation

$$
\Delta_{g} u_{\alpha}+h_{\alpha} u_{\alpha}=u_{\alpha}^{\frac{n+2}{n-2}}
$$

avec $h_{\alpha} \rightarrow h_{0}$ dans $C^{0, \eta}(M)$ pour un certain $\eta>0$. Alors il existe $C>0$ telle que, après extraction d'une sous-suite,

$$
\left|u_{\alpha}(x)-u_{0}(x)\right| \leq C \sum_{i=1}^{N} B_{i, \alpha}(x)+\varepsilon_{\alpha}\left\|u_{0}\right\|_{\infty}
$$

pour tout $x \in M$ où les suites $\left(x_{i, \alpha}\right)_{i=1, \ldots, N}$ et $\left(\mu_{i, \alpha}\right)_{i=1, \ldots, N}$ et la fonction $u_{0}$ sont données par la proposition 3.1 et où $\left(\varepsilon_{\alpha}\right)$ est une suite de réels strictement positifs tendant vers 0 quand $\alpha \rightarrow+\infty$.

Ordonnons les points de concentration par ordre décroissant de hauteur :

$$
\mu_{\alpha}=\mu_{1, \alpha} \geq \mu_{2, \alpha} \geq \cdots \geq \mu_{N, \alpha} .
$$

L'estimée de la proposition s'obtient par récurrence sur $k$ en montrant successivement que les estimées suivantes ont lieu :

$$
\begin{gathered}
\left|u_{\alpha}(x)-u_{0}(x)\right| \\
\leq C\left(\sum_{i=1}^{k} B_{i, \alpha}(x)+\mu_{k, \alpha}^{\frac{n-2}{2}}\left(\min _{i=k+1, \ldots, N}\left(d_{g}\left(x, x_{i, \alpha}\right)+\mu_{i, \alpha}\right)\right)^{2-n}\right)+\varepsilon_{\alpha}\left\|u_{0}\right\|_{\infty} .
\end{gathered}
$$


Il faut remarquer que cette estimée pour $k=1$ est exactement celle de la proposition 3.2 et que, pour $k=n$, nous obtenons l'estimée désirée. Comme dans la sous-section précédente, le cas $u_{0} \not \equiv 0$ est techniquement plus délicat que le cas où la limite faible est nulle. Mais même dans ce cas, des difficultés apparaissent. L'idée naturelle est d'imiter la preuve de l'estimée pour $k=1$ pour passer de $k$ à $k+1$, i.e. passer par des estimées à $\varepsilon$ près grâce au principe du maximum et au point (d) de la proposition 3.1 en choisissant des fonctions-tests adéquates puis d'utiliser la formule de représentation de Green pour passer à l'estimée avec $\varepsilon=0$. Ce schéma fonctionne à quelques modifications d'importance près. Il est quasiimpossible de trouver les bonnes fonctions-tests qui donnent l'estimée à $\varepsilon$ près attendue. Le principe du maximum ne donne qu'une estimée à $\varepsilon$ près a priori moins bonne que celle voulue. La formule de représentation de Green donne alors une estimée (avec $\varepsilon=0$ ) un peu moins bonne que celle voulue (en gros, $\mu_{k+1, \alpha}$ est remplacée par une quantité un peu plus grande). Il faut donc une étape supplémentaire pour montrer que cette quantité peut être en fait prise égale à $\mu_{k+1, \alpha}$. Ce raisonnement par récurrence sur les points de concentration constitue le coeur de la preuve (et sa partie la plus longue et technique). Même si, par rapport à [42], elle a été légèrement simplifiée dans [41] ${ }^{10}$, il paraît illusoire d'espérer la résumer en quelques pages. Nous renvoyons donc à [42] et [41] pour la preuve.

\subsubsection{Asymptotique précise}

Une fois l'estimée optimale par au-dessus obtenue, l'asymptotique précise du théorème 3.1 est relativement simple à obtenir. Il suffit d'écrire avec la formule de représentation de Green que

$$
\begin{aligned}
u_{\alpha}\left(x_{\alpha}\right)-u_{0}\left(x_{\alpha}\right)= & \int_{M} \mathcal{G}\left(x_{\alpha}, x\right)\left(u_{\alpha}(x)^{\frac{n+2}{n-2}}-u_{0}(x)^{\frac{n+2}{n-2}}\right) d v_{g}(x) \\
& +\int_{M} \mathcal{G}\left(x_{\alpha}, x\right)\left(h_{0}(x)-h_{\alpha}(x)\right) u_{\alpha}(x) d v_{g}(x)
\end{aligned}
$$

pour toute suite $\left(x_{\alpha}\right)$ de points de $M$ où $\mathcal{G}$ est la fonction de Green de l'opérateur $\Delta_{g}+h_{0}$ et d'estimer les différents termes grâce à la proposition 3.3 et au point (b) de la proposition 3.1. Nous ne le ferons pas dans ce texte et renvoyons à $[42]$ pour les détails.

10. En particulier, [42] contenait une hiérarchisation plus compliquée des points de concentration (en construisant un arbre généalogique de bulles, où les descendants d'une bulle étaient les bulles qui se trouvaient au-dessus de celle-ci) dont [41] se passe en grande partie. 


\subsubsection{Conclusion}

Résumons brièvement cette preuve. Tout d'abord, il faut trouver un bon changement d'échelle, une bonne renormalisation de la suite de fonctions au voisinage d'un point où elle explose, et ce afin d'obtenir un profil limite. L'étape suivante consiste à trouver une estimée ponctuelle invariante par ce même changement d'échelle qui va permettre de détecter, en deux temps, l'ensemble des points de concentration. La partie la plus longue et la plus technique de la preuve consiste ensuite en la transformation de l'estimée ponctuelle faible en estimée ponctuelle forte. Même si les outils (principe du maximum et fonction de Green) sont simples à décrire, leur agencement n'est pas évident à cause de l'imbrication des bulles les unes dans les autres.

Le théorème 3.1 donne donc une description précise dans l'espace $C^{0}$ de toute suite de solutions d'équations de type Yamabe qui explose. Il est à ce point de vue optimal. Il resterait bien entendu à comprendre de quelles façons les diverses bulles peuvent interagir en fonction du potentiel $h_{\alpha}$, de la géométrie de la variété et de la dimension. Ceci sera l'objet de la section 3.3 .

\subsection{Et si l'opérateur a un noyau?}

A titre de remarque, l'hypothèse que l'opérateur limite $\Delta_{g}+h$ ait un noyau trivial est nécessaire pour obtenir la description ponctuelle du théorème 3.1. En effet, comme démontré dans l'appendice $B$ de [42], si une suite $\left(u_{\alpha}\right)$ de solutions strictement positives de

$$
\Delta_{g} u_{\alpha}+h_{\alpha} u_{\alpha}=u_{\alpha}^{\frac{n+2}{n-2}}
$$

avec $h_{\alpha} \rightarrow h_{0}$ dans $C^{0, \eta}(M)$ vérifie

$u_{\alpha}\left(x_{\alpha}\right)=(1+o(1)) u_{0}\left(x_{\alpha}\right)+\sum_{i=1}^{N}(1+o(1)) B_{i, \alpha}\left(x_{\alpha}\right)+O\left(\left(\max _{i=1, \ldots, N} \mu_{i, \alpha}\right)^{\frac{n-2}{2}}\right)$

pour toute suite de points $\left(x_{\alpha}\right)$, alors l'opérateur $\Delta_{g}+h_{0}$ est coercif et en particulier a un noyau trivial.

\subsection{Rayons d'influence des bulles}

La notion essentielle pour obtenir des informations sur les configurations de bulles possibles et démontrer le théorème 2.1 est celle de rayon d'influence d'une bulle. Etant donné $1 \leq i \leq N$, le rayon d'influence de la 
$i$-ème bulle est défini par

$r_{i, \alpha}= \begin{cases}\min _{j \in \mathcal{A}_{i}} \sqrt{\frac{\mu_{i, \alpha}}{\mu_{j, \alpha}} d_{g}\left(x_{i, \alpha}, x_{j, \alpha}\right)^{2}+\mu_{i, \alpha} \mu_{j, \alpha}} & \text { si } u_{0} \equiv 0 \\ \min \left\{\min _{\left.j \in \mathcal{A}_{i} \sqrt{\frac{\mu_{i, \alpha}}{\mu_{j, \alpha}} d_{g}\left(x_{i, \alpha}, x_{j, \alpha}\right)^{2}+\mu_{i, \alpha} \mu_{j, \alpha}} ; \sqrt{\mu_{i, \alpha}}\right\}}\right. & \text { si } u_{0} \neq \equiv\end{cases}$

où

$$
\mathcal{A}_{i}=\left\{1 \leq j \leq N, j \neq i \text { t.q. } \mu_{i, \alpha}=O\left(\mu_{j, \alpha}\right)\right\}
$$

Evidemment, si $\mathcal{A}_{i}=\emptyset$ et $u_{0} \equiv 0$, nous poserons $r_{i, \alpha}=\frac{1}{2} i_{g}(M)$ par exemple. Derrière cette définition d'apparence compliquée se cache en fait une idée simple : $r_{i, \alpha}$ doit être vu comme le rayon de la boule de centre $x_{i, \alpha}$ dans laquelle la bulle $B_{i, \alpha}$ est dominante dans la décomposition (2.4), excepté que doivent être oubliées les bulles qui sont beaucoup plus hautes qu'elle ${ }^{11}$. Les rayons d'influence de différentes bulles sont représentés sur la figure 6 , page 54 .

11. C'est le sens de la restriction $j \in \mathcal{A}_{i}$. De toutes façons, ces bulles qui sont beaucoup plus hautes, si jamais elles étaient centrées dans la boule de centre $x_{i, \alpha}$ et de rayon $r_{i, \alpha}$, ne seraient beaucoup plus hautes que $B_{i, \alpha}$ que sur une boule de rayon négligeable devant $r_{i, \alpha}$. 


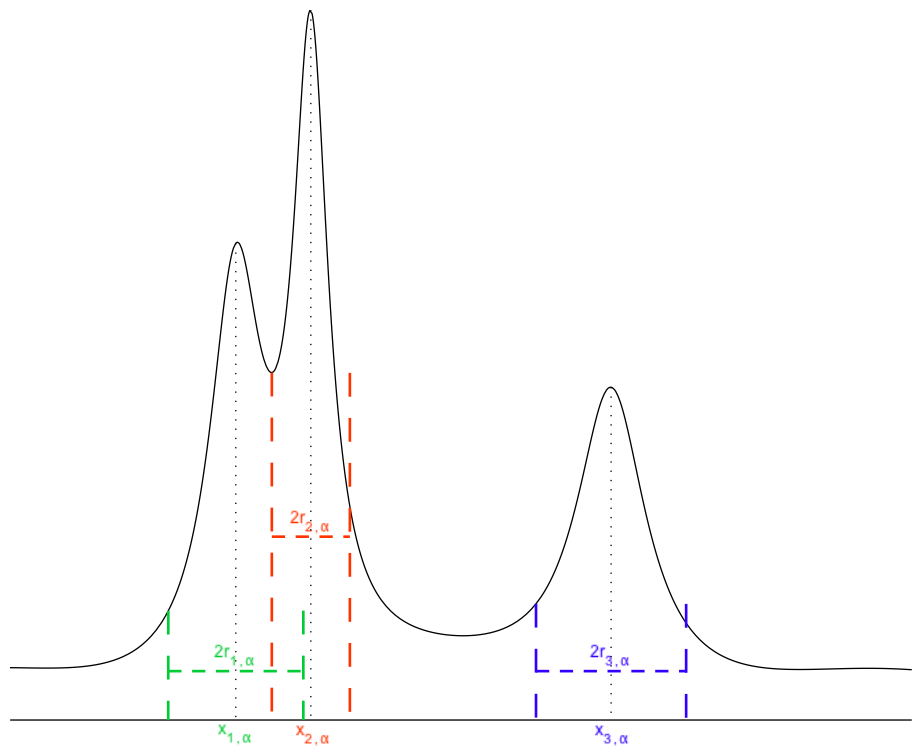

Fig. 6 : rayons d'influence des bulles.

Pourquoi s'intéresser au rayon d'influence d'une bulle? Tout simplement parce que c'est à cette échelle, i.e. à distance de l'ordre de $r_{i, \alpha}$ de $x_{i, \alpha}$, que l'interaction ponctuelle entre cette bulle et une autre (ou $u_{0}$ ) est la plus forte. C'est donc à cette distance que va se produire le recollement entre cette bulle et une autre bulle (ou $u_{0}$ ). Or recoller une bulle sur une bulle (ou sur une fonction $u_{0}$ ) tout en restant solution de l'équation est nécessairement contraignant. C'est donc en comprenant précisément ce qui se passe à cette échelle que nous pouvons espérer obtenir de précieuses informations sur les positions relatives des bulles les unes par rapport aux autres. Enfin, si le rayon d'influence ne tend pas vers 0, ce qui n'arrive que lorsque la bulle est l'une des moins hautes et $u_{0} \equiv 0$, la géométrie de la variété devrait jouer un rôle dans le recollement de cette bulle pour donner une solution globale de l'équation.

En travaillant par récurrence de la bulle la plus haute à la plus basse, il est possible de démontrer que, pour tout $1 \leq i \leq N$, si $r_{i, \alpha} \rightarrow 0$ quand 
$\alpha \rightarrow+\infty$

$$
\left(h\left(x_{i}\right)-\frac{1}{6} S_{g}\left(x_{i}\right)+o(1)\right) r_{i, \alpha}^{2} \ln \frac{r_{i, \alpha}}{\mu_{i, \alpha}}=C_{i}
$$

si $n=4$ et

$$
\left(h\left(x_{i}\right)-\frac{1}{6} S_{g}\left(x_{i}\right)+o(1)\right) r_{i, \alpha}^{n-2} \mu_{i, \alpha}^{4-n}=C_{i}
$$

si $n \geq 5$ où $C_{i}$ est une constante strictement positive (qui est explicite et dépend de l'endroit où se trouve les autres bulles et de $\left.u_{0}\right)$. En dimension 3 , il est en fait impossible de coller des bulles sur des bulles et $r_{i, \alpha} \nrightarrow \rightarrow 0$ quand $\alpha \rightarrow+\infty$. Nous renvoyons à [35] pour la preuve de ces estimées sur les rayons d'influence.

\subsection{Preuve du théorème 2.1}

Ainsi le recollement doit se faire à une distance précise, qui dépend de la hauteur de la bulle. De plus, il est clair qu'une bulle ne peut être recollée sur une autre bulle (ou sur une fonction $u_{0}$ ) que si, au centre de la bulle, $h\left(x_{i}\right) \geq \frac{n-2}{4(n-1)} S_{g}\left(x_{i}\right)$.

Mais ceci ne nous donne pas encore le résultat : il est possible de recoller une bulle sur une autre bulle même si $h>\frac{n-2}{4(n-1)} S_{g}$. C'est la bulle la plus basse qui, elle, doit absolument se concentrer en un point où $h(x)=$ $\frac{n-2}{4(n-1)} S_{g}(x)$. Il faut là encore distinguer plusieurs cas : cette bulle est isolée; cette bulle doit se recoller sur une limite faible non-nulle; cette bulle arrive dans un chapelet de bulles de taille équivalente et de centres très rapprochés les uns des autres.

Reprenons ces différents cas. Par bulle isolée, nous signifions ici simplement que $r_{i, \alpha} \nrightarrow \rightarrow 0$ quand $\alpha \rightarrow+\infty$. Ceci ne veut pas dire qu'il n'y a pas des bulles beaucoup plus hautes qui se concentrent au même endroit mais cela exclut l'existence d'une limite faible non-nulle et la présence de bulles de hauteur équivalente se concentrant au même endroit. Dans ce cas, la conclusion vient assez directement de l'application d'une identité de Pohožaev : la bulle doit se concentrer à un endroit où $h(x)=\frac{n-2}{4(n-1)} S_{g}(x)$ en dimensions $n \geq 4$. La situation en dimension 3 est plus compliquée et nous ne la discuterons pas ici.

Si la bulle doit se recoller sur une limite faible non-nulle, i.e. $r_{i, \alpha}=$ $\sqrt{\mu_{i, \alpha}}$, l'estimation sur les rayons d'influence donne une contradiction en dimensions $n=3,4,5$ et donne directement que $h\left(x_{i}\right)=\frac{n-2}{4(n-1)} S_{g}\left(x_{i}\right)$ si $n \geq 7$. Par contre, a priori, rien ne l'interdit en dimension 6 mais alors l'estimée des rayons d'influence nous dit, de façon surprenante, que $h\left(x_{i}\right)>$ $\frac{n-2}{4(n-1)} S_{g}\left(x_{i}\right)$. A titre de remarque, l'estimée sur les rayons d'influence donne $r_{i, \alpha} \gg \sqrt{\mu_{i, \alpha}}$ et donc la nullité de $u_{0}$ en dimensions 3,4 et 5 , ce qui 
signifie que la suite $\left(u_{\alpha}\right)$ ne peut pas développer de points de concentration si sa limite faible est non-nulle.

Enfin, si cette bulle arrive avec un chapelet de bulles de même taille qui se concentrent toutes au même endroit, c'est un argument de balance qui donne la contradiction. Pour que cela soit possible, il faudrait que chaque centre de bulle soit le "centre de gravité" des autres centres des bulles du chapelet pondérées par leur paramètre $\mu_{i, \alpha}$. Mais, comme le nombre de bulles est fini, il y en a toujours une qui se trouve le plus à l'extérieur, d'où une contradiction. Bref, cette situation est impossible, quelque soit la dimension ${ }^{12}$. Pour plus de détails, nous renvoyons à [35].

Revenons sur les résultats de l'analyse contenue dans [35]. Tout d'abord, il a été démontré qu'une limite faible non-nulle en dimensions $n=3,4,5$ interdisait tout phénomène de concentration. Dans ces petites dimensions, il est impossible d'avoir à la fois $u_{0} \not \equiv 0$ et $N \geq 1$ dans la décomposition (2.4). Résultat surprenant s'il en est. En dimension 3, et c'est un phénomène du même ordre, il ne peut y avoir accumulation de bulles : les points de concentration sont isolés. En effet, la morale de cette histoire est la suivante : plus les dimensions sont petites, plus il est difficile de recoller des bulles entre elles; plus une bulle est étalée et basse, plus elle est difficile à recoller à une autre de hauteur donnée. Par "difficile à recoller", nous entendons que le recollement doit avoir lieu plus loin du point de concentration. Il suffit maintenant de voir la limite faible non-nulle comme une bulle de poids 1 , i.e. maximalement étalée pour éclairer un peu le premier résultat à la lumière du second.

Les résultats du chapitre 2 sont tous des conséquences de cette estimée sur les rayons d'influence des bulles.

\subsection{Et si l'énergie n'est pas bornée?}

Lorsqu'aucune hypothèse sur l'énergie n'est faite, une telle théorie asymptotique ponctuelle n'est pas connue, pour la simple et bonne raison que la suite $\left(u_{\alpha}\right)$ peut développer une infinité de points de concentration. De telles suites, solutions d'équations proches de celle considérée ici, ont été construites par Wei et ses collaborateurs, voir par exemple [99, 100].

Démontrer le théorème 2.2 utilise une approche différente de celle utilisée pour la preuve du théorème 2.1. En effet, dès que $h_{0}<\frac{n-2}{4(n-1)} S_{g}$, il est possible de raisonner à partir de la bulle la plus haute et de démontrer que celle-ci ne peut pas apparaître. La contradiction vient donc de la bulle la plus haute. En effet, recoller une bulle sur une autre bulle ou sur une limite faible non nulle nécessite, en tout cas en dimensions $n \geq 4$, d'avoir

12. Ici, l'hypothèse d'énergie finie, et sa conséquence qui est que le nombre de bulles est fini, est cruciale. 
$h_{0} \geq \frac{n-2}{4(n-1)} S_{g}$ au point de concentration ${ }^{13}$. En dimension 3, la situation est un petit peu différente et les bulles sont nécessairement isolées. Enfin, pour recoller une bulle sur la variété, il faut avoir $h_{0}=\frac{n-2}{4(n-1)} S_{g}$ au point de concentration en dimensions $n \geq 4$ et la masse de la fonction de Green nulle en ce point en dimension 3. Dans la preuve du théorème 2.1 , c'est la bulle la plus basse qui donne la contradiction. Rien n'interdit de recoller une bulle sur une bulle lorsque $h_{0}>\frac{n-2}{4(n-1)} S_{g}$ mais la bulle la plus basse, qui doit être recollée soit sur la limite faible, soit sur la variété, ne peut l'être que si $h_{0}=\frac{n-2}{4(n-1)} S_{g}$, sauf en dimensions 3 et 6 .

Ainsi ces deux approches sont vraiment différentes. Pour démontrer la compacité dans l'équation de Yamabe, c'est par exemple l'approche sans borne sur l'énergie qui est utilisée; il faut donc démontrer directement qu'il est impossible de recoller une bulle sur quoique ce soit. Quand la contradiction vient de la bulle la plus basse, il semble délicat de la détecter lorsqu'aucune borne sur l'énergie n'est faite a priori. Mais il est également extrêmement délicat de construire des suites de solutions qui explosent avec une énergie tendant vers $+\infty$, c'est-à-dire développant une infinité de bulles.

13. Voir les estimations sur les rayons d'influence de la page 55. 


\section{Chapitre 4}

\section{D'autres équations}

Nous allons passer en revue un certain nombre d'équations pour lesquelles des résultats de stabilité ont été obtenus. Par la même occasion, nous allons sur quelques exemples montrer comment la théorie asymptotique ponctuelle du chapitre 3 s'adapte à des équations plus compliquées que l'équation de type Yamabe.

\subsection{L'équation d'Einstein-Lichnerowicz}

Dans [40], nous avons étudié cette question de stabilité pour les équations d'Einstein-Lichnerowicz avec champ scalaire sur des variétés riemanniennes compactes. Ces équations proviennent des équations de contrainte issues de la théorie de la relativité générale, plus précisément lorsque la gravitation est couplée avec un champ scalaire vérifiant une équation des ondes non-linéaire (ou linéaire).

Dans la première sous-section ci-dessous, nous allons décrire l'origine de l'équation dont nous étudierons la stabilité dans la sous-section suivante. Nous expliquerons également pourquoi il est intéressant d'obtenir des résultats de stabilité forte pour cette équation.

\subsubsection{Les contraintes dans la théorie d'Einstein avec champ scalaire}

Dans la théorie de la relativité générale, l'espace-temps est décrit par une variété lorentzienne $(X, h)$ de dimension $n+1$. L'équation d'Einstein relie courbure de cet espace-temps et matière par

$$
R_{i j}-\frac{1}{2} S h_{i j}=8 \pi \mathcal{G} T_{i j}
$$


Dans cette équation, $R_{i j}$ est la courbure de Ricci de $(X, h), S$ sa courbure scalaire et $T_{i j}$ est le tenseur énergie-impulsion de la matière présente, $\mathcal{G}$ étant la constante gravitationnelle de Newton. La forme du tenseur $T_{i j}$ reste à déterminer et dépend du modèle utilisé pour décrire la matière. Toujours est-il que, pour des raisons de conservation de l'énergie, celui-ci doit être à divergence nulle, i.e. $T_{i j}{ }^{j}=0$, ce qui est d'ailleurs clair pour des raisons géométriques une fois posée l'équation d'Einstein ${ }^{1}$ puisque $\left(R_{i j}-\frac{1}{2} S h_{i j}\right)^{, j}=0$.

Dans une théorie de champ scalaire, le tenseur $T$ dépend de la métrique $h$ et d'un champ scalaire $\psi: X \mapsto \mathbb{R}$ et s'écrit

$$
T_{i j}=\partial_{i} \psi \partial_{j} \psi-\left(\frac{1}{2}|\nabla \psi|_{h}^{2}+V(\psi)\right) h_{i j}
$$

où $V$ est un potentiel. Comme $T$ est à divergence nulle, le champ $\psi$ doit vérifier une équation des ondes

$$
\nabla^{i} \partial_{i} \psi=\frac{d V}{d \psi} .
$$

Le potentiel dépend de la théorie considérée : pour un champ de KleinGordon massif, $V(\psi)=\frac{1}{2} m \psi^{2}$; le cas $V(\psi) \equiv \Lambda$ et $\psi \equiv 0$ redonne l'équation d'Einstein dans le vide avec une constante cosmologique. Dans la suite, nous oublierons la constante physique $8 \pi \mathcal{G}$ dans l'équation d'Einstein et celle-ci s'écrira donc dans notre situation

$$
R_{i j}-\frac{1}{2} S h_{i j}=\partial_{i} \psi \partial_{j} \psi-\left(\frac{1}{2}|\nabla \psi|_{h}^{2}+V(\psi)\right) h_{i j} .
$$

Considérons maintenant une tranche d'espace $M$, i.e. une hypersurface (de dimension $n$ ) de type espace ${ }^{2}$ dans $X$. Ecrivons localement la métrique sous la forme

$$
h=-N^{2} d t \otimes d t+\gamma_{i j} \theta^{i} \otimes \theta^{j}
$$

où $\left(d t, d \theta^{i}\right)$ est la base duale de la base associée à la tranche d'espace choisie $\left(e_{\perp}=\frac{1}{N}\left(\frac{\partial}{\partial t}-\beta^{j}\left(\frac{\partial}{\partial x_{j}}\right)\right), \frac{\partial}{\partial x_{1}}, \ldots, \frac{\partial}{\partial x_{n}}\right)$. La fonction $N$ est la fonction de délai, les $\beta^{j}$ sont les composantes du vecteur décalage. Ces deux données dépendent du feuilletage local de $X$ et de la fonction temps choisis. Posons pour la suite

$$
\pi=N^{-1}\left(\frac{\partial \psi}{\partial t}-\beta^{j} \frac{\partial \psi}{\partial x_{j}}\right) .
$$

1. Mais c'est ce qui s'appelle renverser le processus historique puisque c'est entre autres cette conservation de l'énergie qui a conduit Einstein à ce choix de tenseur géométrique dans son équation.

2. "de type espace" signifie que la métrique induite de $h$ sur $M$ est définie positive, i.e. riemannienne. 
En utilisant l'équation d'Einstein pour $G_{\perp \perp}$ et l'équation de Gauss, nous obtenons la contrainte hamiltonienne

$$
2 N^{-2} G_{\perp \perp}=S_{\gamma}-|K|_{\gamma}^{2}+\left(t r_{\gamma} K\right)^{2}=\pi^{2}+|\nabla \psi|_{\gamma}^{2}+2 V(\psi)
$$

où $S_{\gamma}$ est la courbure scalaire de la variété riemannienne $(M, \gamma)$ et $K$ est la seconde forme fondamentale de la sous-variété $M$ dans $X$. De l'équation d'Einstein sur les $G_{j \perp}$ et des équations de Codazzi, nous obtenons la contrainte de moment

$$
-N^{-1} G_{j \perp}=K_{j m}{ }^{, m}-\partial_{j}\left(\operatorname{tr}_{\gamma} K\right)=\pi \partial_{j} \psi .
$$

Ces équations dites de contrainte portent sur $\gamma, K, \psi$ et $\pi$ mais ne contiennent pas $N$ et $\beta$.

Le reste de l'équation d'Einstein (i.e. portant sur les $G_{i j}$ ) donne alors les équations d'évolution

$$
\begin{aligned}
\frac{\partial K_{i j}}{\partial t}= & N\left(R_{i j}-2 K_{i m} K_{j}^{m}+\left(t r_{\gamma} K\right) K_{i j}-\partial_{i} \psi \partial_{j} \psi+\frac{1}{n-1} V(\psi) \gamma_{i j}\right) \\
& -N^{-1} N_{, i j}+\mathcal{L}_{\beta} K_{i j}
\end{aligned}
$$

et

$$
\frac{\partial \pi}{\partial t}=N\left(\Delta \psi+\left(\operatorname{tr}_{\gamma} K\right) \pi-\frac{\partial V}{\partial \psi}\right)+\gamma^{k l} \partial_{k} N \partial_{l} \psi+\mathcal{L}_{\beta} \pi .
$$

Les données $\psi$ et $\gamma$ évoluent par

$$
\frac{\partial \psi}{\partial t}=N \pi+\mathcal{L}_{\beta} \pi
$$

et

$$
\frac{\partial \gamma_{i j}}{\partial t}=-2 N K_{i j}+\mathcal{L}_{\beta} \gamma_{i j}
$$

grâce aux définitions de $\pi$ et $K$. Les variables $N$ et $\beta$ sont complètement libres durant l'évolution. Cela reflète l'invariance des équations sous l'action du groupe des difféomorphismes.

Dans [26], en s'inspirant de [46] et en s'appuyant sur les travaux de Leray [66], il est démontré que le système d'équations (4.4) et (4.5) est hyperbolique et bien posé ${ }^{3}$, quitte à faire un bon choix de jauge ${ }^{4}$. Partant d'une variété riemannienne $(M, \gamma)$ et de données $K, \psi$ et $\pi$ sur $M$ satisfaisant les équations de contrainte (4.2)-(4.3), le système d'équations d'évolution (4.4)-(4.5) admet une solution en temps petit sur $M$ avec les conditions initiales $(\gamma, K, \psi, \pi)$. En d'autres termes, il existe une variété lorentzienne $X$

3. Pour les équations d'Einstein dans le vide, ce résultat remonte aux années 50 et au travail de Choquet-Bruhat [46].

4. i.e. de fonctions $N$ et $\beta$. 
vérifiant l'équation d'Einstein (4.1) telle que $M$ soit une tranche d'espace dans $X, \gamma$ soit la métrique induite sur $M$ par la métrique lorentzienne, $K$ soit sa seconde forme fondamentale, $\psi$ et $\pi$ soient les données du champ et de sa "dérivée temporelle" sur $M$.

Trouver des solutions des équations de contrainte permet donc de générer des espaces-temps vérifiant les équations d'Einstein avec champ scalaire. Comprendre l'ensemble des solutions des équations de contrainte peut également avoir un intérêt puisque ces contraintes sont bien sûr vérifiées tout au long de l'évolution. Pour un très bel article de survol sur ces équations de contrainte, nous renvoyons à [9]. Y est en particulier expliquée en détails la méthode dite conforme pour trouver des solutions à ces équations de contrainte, méthode que nous allons brièvement décrire dans notre situation maintenant.

Les équations de contrainte (4.2)-(4.3) sont sous-déterminées ${ }^{5}$. Assez paradoxalement, un bon moyen pour trouver des solutions à ces équations est de fixer un certain nombre d'inconnues afin de rendre le système déterminé et de tenter de résoudre ce système pour les inconnues restantes. C'est l'objet de la méthode conforme, initiée par Lichnerowicz [71]. Partons d'une variété riemannienne $(M, g)$. Considérons comme fixée la classe conforme ${ }^{6}$ et cherchons $\gamma$ sous la forme

$$
\gamma=\varphi^{\frac{4}{n-2}} g
$$

pour une fonction $\varphi$ régulière et strictement positive sur $M$. Puisque les courbures scalaires de $\gamma$ et de $g$ sont reliées par l'équation

$$
\Delta_{g} \varphi+\frac{n-2}{4(n-1)} S_{g} \varphi=\frac{n-2}{4(n-1)} S_{\gamma} \varphi^{\frac{n+2}{n-2}},
$$

la contrainte hamiltonienne (4.2) devient

$$
\begin{aligned}
\varphi^{-\frac{n+2}{n-2}}\left(\frac{4(n-1)}{n-2} \Delta_{g} \varphi+S_{g} \varphi\right)= & \pi^{2}+\varphi^{-\frac{4}{n-2}}|\nabla \psi|_{g}^{2}+2 V(\psi) \\
& +\varphi^{-\frac{8}{n-2}}|K|_{g}^{2}-\tau^{2}
\end{aligned}
$$

avec

$$
\tau=\gamma^{i j} K_{i j} .
$$

La contrainte de moment (4.3) devient quant à elle

$$
\varphi^{-\frac{4}{n-2}}\left(\operatorname{div}_{g} K\right)_{i}-\frac{2}{n-2} \tau \varphi^{-1} \varphi_{, i}+2 \varphi^{-\frac{n+2}{n-2}} K_{i j} \varphi^{, j}=\pi \psi_{, i}+\tau_{, i} .
$$

5. Il y a $n+1$ équations pour $n^{2}+n+2$ inconnues.

6. Cela élimine déjà $\frac{n^{2}+n-2}{2}$ variables. 
Cette deuxième équation sur $K$ se simplifie en posant

$$
K=\varphi^{-2} P+\frac{\tau}{n} \varphi^{\frac{4}{n-2}} g
$$

avec $P$ sans trace. Elle devient alors

$$
\varphi^{-\frac{2 n}{n-2}} \operatorname{div}_{g}(P)_{i}=\frac{n-1}{n} \tau_{, i}+\pi \psi_{, i}
$$

Celle-ci est encore sous-déterminée. Considérons $\tau$, qui sera la courbure moyenne de $M$ dans l'espace-temps $X$, fixé, et posons également ${ }^{7}$

$$
P=U+\mathcal{L}_{g} X
$$

pour $U$ un champ de $(2,0)$-tenseurs sans trace et à divergence nulle et $X$ un champ de vecteurs. Ici, $\mathcal{L}_{g} X$ est la dérivée de Lie conforme de $X$, i.e.

$$
\left(\mathcal{L}_{g} X\right)_{i j}=X_{i, j}+X_{j, i}-\frac{2}{n}\left(\operatorname{tr}_{g} X\right) g_{i j}
$$

Si $U$ est considérée comme donnée, le nombre d'inconnus devient égal au nombre d'équations puisque la contrainte de moment devient

$$
\varphi^{-\frac{2 n}{n-2}} \operatorname{div}_{g}\left(\mathcal{L}_{g} X\right)_{i}=\frac{n-1}{n} \tau_{, i}+\pi \psi_{, i} .
$$

Nous arrivons donc au système suivant :

$$
\begin{aligned}
\frac{4(n-1)}{n-2} \Delta_{g} \varphi+\left(S_{g}-|\nabla \psi|_{g}^{2}\right) \varphi= & \left(2 V(\psi)-\frac{n-1}{n} \tau^{2}+\pi^{2}\right) \varphi^{\frac{n+2}{n-2}} \\
& +\varphi^{-\frac{3 n-2}{n-2}}\left|U+\mathcal{L}_{g} X\right|_{g}^{2}
\end{aligned}
$$

et

$$
\operatorname{div}_{g}\left(\mathcal{L}_{g} X\right)_{i}=\left(\frac{n-1}{n} \tau_{, i}+\pi \psi_{, i}\right) \varphi^{\frac{2 n}{n-2}}
$$

d'inconnues $\varphi$ et $X$. Afin de le découpler au maximum, il convient de poser

$$
\pi=\varphi^{-\frac{2 n}{n-2}} \tilde{\pi}
$$

pour arriver au système final :

$$
\left\{\begin{array}{l}
\Delta_{g} \varphi+h \varphi=f \varphi^{\frac{n+2}{n-2}}+a \varphi^{-\frac{3 n-2}{n-2}} \\
\Delta_{g, \text { conf }} X=\frac{n-1}{n} \varphi^{\frac{2 n}{n-2}} \nabla \tau+\tilde{\pi} \nabla \psi
\end{array}\right.
$$

7. Tout champ de $(2,0)$-tenseurs symétriques sans trace peut se décomposer ainsi. 
avec

$$
\begin{aligned}
h & =\frac{n-2}{4(n-1)}\left(S_{g}-|\nabla \psi|_{g}^{2}\right), \\
f & =\frac{n-2}{4(n-1)}\left(2 V(\psi)-\frac{n-1}{n} \tau^{2}\right) \text { et } \\
a & =\frac{n-2}{4(n-1)}\left(\left|U+\mathcal{L}_{g} X\right|_{g}^{2}+\tilde{\pi}^{2}\right) .
\end{aligned}
$$

Etant donnés une variété riemannienne $(M, g)$, des fonctions $\psi, \tilde{\pi}, \tau$ sur $M$ et $U$ un champ de $(2,0)$-tenseurs sans trace à divergence nulle, si $X$ et $\varphi$ vérifient le système d'équations (4.6), alors les données $(M, \gamma), K, \psi$ et $\pi$ avec

$$
\gamma=\varphi^{\frac{4}{n-2}} g, \pi=\varphi^{-\frac{2 n}{n-2}} \tilde{\pi} \text { et } K=\varphi^{-2}\left(U+\mathcal{L}_{g} X\right)+\frac{\tau}{n} \varphi^{\frac{4}{n-2}} g
$$

satisfont les contraintes hamiltonienne (4.2) et de moment (4.3) et constituent donc des données initiales pour le problème de Cauchy ${ }^{8}$.

Ce système est entièrement découplé si $\tau$ est constante ${ }^{9}$. Dans ce cas, il suffit de résoudre la seconde équation, ce qui ne pose aucun problème puisque l'opérateur $\Delta_{g, \text { conf }}$ est elliptique. Il faut juste s'assurer que le noyau de cet opérateur est trivial (ou orthogonal à la donnée $\tilde{\pi} \nabla \psi$ ). Les éléments du noyau sont des champs de Killing conformes et leur présence est rare d'après [10]. Une fois résolue la seconde équation, reste à utiliser $X$ comme donnée supplémentaire dans la première et à trouver une solution $\varphi$ à celle-ci.

La méthode principale qui a été utilisée pour résoudre ce système (dans le cas découplé) est la méthode des sur- et sous-solutions. Celle-ci donne des résultats lorsque la fonction $f$ est strictement négative. Par exemple, dans [27], il est démontré que la première équation de (4.6) admet une solution dès que $f \leq 0, a>0$ et l'opérateur $\Delta_{g}+h$ est coercif. A titre de remarque, dans le cas découplé, pour les équations d'Einstein dans le vide, i.e. avec $\psi=0, \pi=0$ et $V \equiv 0$, il est complètement connu, en fonction des données, si l'équation admet des solutions ou non (voir Bartnik-Isenberg [9]). Plus récemment, Hebey-Pacard-Pollack [55] ont utilisé une méthode variationnelle pour démontrer des résultats d'existence sur le système découplé mais cette fois en accordant que $f$ soit positive ou change de signe.

8. En particulier, il existe un espace-temps $X=(-\varepsilon, \varepsilon) \times M$ vérifiant l'équation d'Einstein (4.1) avec $\gamma$ la métrique induite sur $M, K$ la seconde forme fondamentale de $M$ dans $X$, et donc $\tau$ sa courbure moyenne, $\psi$ et $\pi$ étant les données du champ et de sa dérivée temporelle sur la tranche d'espace $M$.

9. Cette fonction $\tau$ est la courbure moyenne de l'hypersurface $M$ dans son développement de Cauchy $X=M \times(-\varepsilon ; \varepsilon)$. 
Pour le système couplé, presque rien n'est connu. Pour les équations d'Einstein dans le vide, la seule approche est encore par sur- et soussolutions, notions développées pour les systèmes dans [60]. Cette approche a été raffinée par Maxwell dans [78]. Mais il est certain que ces techniques achopperont sur le signe de la non-linéarité $f$ pour notre système (4.6). Nous sommes pour notre part convaincus que la méthode variationnelle, couplée avec des résultats de stabilité, devrait donner des résultats dans le cas couplé. La sous-section suivante peut être vue comme une première étape vers cet objectif.

\subsubsection{Stabilité et instabilité}

Soit l'équation de Lichnerowicz de la sous-section précédente, i.e.

$$
\Delta_{g} u+h u=f u^{\frac{n+2}{n-2}}+a u^{-\frac{3 n-2}{n-2}}
$$

où $h, f$ et $a$ sont des fonctions régulières sur une variété riemannienne compacte de dimension $n \geq 3$. Notons, pour $0 \leq \Lambda \leq+\infty$,

$$
\mathcal{S}_{a, h, f}^{\Lambda}=\left\{u \in C^{2}(M) \text { solution de (4.7) avec } \int_{M} u^{\frac{2 n}{n-2}} d v_{g} \leq \Lambda\right\} .
$$

Remarquons que $\mathcal{S}_{a, h, f}^{\infty}$ n'est rien d'autre que l'ensemble des solutions de (4.7). L'équation (4.7) sera dite stable si pour tous $\Lambda>0$ et $\varepsilon>0$, il existe $\eta>0$ tel que

$$
\left\|a-a^{\prime}\right\|_{C^{0}}+\left\|h-h^{\prime}\right\|_{C^{0}}+\left\|f-f^{\prime}\right\|_{C^{2}} \leq \eta \Rightarrow d_{C^{2}}^{\hookrightarrow}\left(\mathcal{S}_{a^{\prime}, h^{\prime}, f^{\prime}}^{\Lambda+\varepsilon}, \mathcal{S}_{a, h, f}^{\Lambda}\right) \leq \varepsilon
$$

où la distance $d_{C^{2}}^{\hookrightarrow}$ a été définie en (1.1), section1.1. Elle sera dite fortement stable si, de plus, l'ensemble $\mathcal{S}_{a, h, f}^{\Lambda}$ est borné dans $C^{2}(M)$ pour tout $\Lambda>0$. Nous dirons également que l'équation est globalement fortement stable si pour tout $\varepsilon>0$, il existe $\eta>0$ tel que

$$
\left\|a-a^{\prime}\right\|_{C^{0}}+\left\|h-h^{\prime}\right\|_{C^{0}}+\left\|f-f^{\prime}\right\|_{C^{2}} \leq \eta \Rightarrow d_{C^{2}}^{\hookrightarrow}\left(\mathcal{S}_{a^{\prime}, h^{\prime}, f^{\prime}}^{\infty}, \mathcal{S}_{a, h, f}^{\infty}\right) \leq \varepsilon
$$

et si l'ensemble $\mathcal{S}_{a, h, f}^{\infty}$ est borné dans $C^{2}(M)$. Le résultat que nous avons démontré dans [40] est le suivant :

Théorème 4.1. [Druet-Hebey, [40]] Soit $(M, g)$ une variété riemannienne compacte de dimension $3 \leq n \leq 5$. Soient $a$, $h$ et $f$ trois fonctions régulières sur $M$. Alors

- l'équation (4.7) est fortement stable dès que $a>0$.

- l'équation (4.7) est globalement fortement stable dès que a $>0$ et $f>0$. 
Un corollaire de ce résultat est qu'en dimensions $3 \leq n \leq 5$, pour des choix génériques de fonctions $a$, $h$ et $f$ avec $a>0$ et $f>0$, il existe un nombre pair, éventuellement nul, de solutions à l'équation (4.7). En particulier, à chaque fois que [55] nous fournit une solution, il en existe une deuxième (sauf cas exceptionnel). Un simple argument de théorie du degré donne ce corollaire à partir du moment où la forte stabilité globale est démontrée pour l'ensemble des données considérées.

Il faut remarquer que l'hypothèse $a>0$ est compatible avec le problème des contraintes décrit ci-dessus (il suffit par exemple de prendre $\tilde{\pi}>0$ ) et que c'est une hypothèse cruciale dans ce théorème ${ }^{10}$. Ainsi l'équation de Lichnerowicz ne pose pas de problèmes d'instabilité dans le système (4.6), au moins en petites dimensions. C'est de bon augure pour qui veut comprendre le système couplé.

En dimensions plus grandes que 6, l'équation n'est pas stable sous les hypothèses du théorème 4.1. En effet, nous avons la proposition suivante, tirée de [40]:

Proposition 4.1. Pour tout $n \geq 6$, il existe des variétés riemanniennes compactes $(M, g)$ de dimension $n$ et des fonctions a et $h$ strictement positives telles que l'équation

$$
\Delta_{g} u+h u=u^{\frac{n+2}{n-2}}+a u^{-\frac{3 n-2}{n-2}}
$$

ne soit pas stable.

L'effet dimensionnel de ces résultats est à relier avec le fait qu'en dimensions $3 \leq n \leq 5$, il est impossible pour une suite de solutions d'équation de type Yamabe d'exploser avec une limite faible non-nulle. Ici le terme en puissance négative dans l'équation (avec le bon signe de $a$ ) assure en quelque sorte que cette limite faible est non-nulle (même si, pour l' $\infty$ stabilité, il n'est même pas certain qu'elle existe). Ensuite, la preuve ressemble dans les deux cas beaucoup plus à une preuve de type "stabilité globale" que de type "stabilité" (cf. discussion de la section 3.5). En fait, il est directement démontré qu'aucun point de concentration ne peut apparaître, sans appel à la bulle la plus basse. L'hypothèse sur l'énergie lorsque $f$ change de signe est là pour éviter des profils de concentration dégénérés.

\subsection{Systèmes d'EDP elliptiques}

Dans [39], [41] et [43], nous nous sommes intéressés avec Emmanuel Hebey et Jérôme Vétois à cette question de stabilité pour des systèmes

10. Il est impossible d'obtenir un résultat de stabilité sans hypothèse sur $h$ et $f$ si $a$ est autorisé à s'annuler voire à changer de signe. 
d'EDP elliptiques. Ces systèmes sont vérifiés par les ondes stationnaires pour des systèmes couplés d'équations de Schrödinger non-linéaires ${ }^{11}$ (de type Gross-Pitaevskii). Ces systèmes apparaissent essentiellement dans deux branches de la physique : dans la théorie de Hartree-Fock des doubles condensats de Bose-Einstein (cf. [21]), dans l'étude des solitons incohérents en optique non-linéaire (cf. [4, 28, 58, 59, 61]).

Soit $(M, g)$ une variété riemannienne compacte de dimension $n \geq 3$. Pour $p \geq 1$ un entier, $M_{p}^{s}(\mathbb{R})$ dénote l'espace vectoriel des matrices carrées symétriques réelles de taille $p$. Considérons le système d'EDP elliptiques

$$
\Delta_{g}^{p} \mathcal{U}+\mathcal{A} \mathcal{U}=\mathcal{U}^{\frac{n+2}{n-2}}
$$

où $\mathcal{A}: M \mapsto M_{p}^{s}(\mathbb{R})$ est $C^{1}, \mathcal{U}: M \mapsto \mathbb{R}^{p}$ est une $p$-fonction, $\Delta_{g}^{p}$ est l'opérateur de Laplace-Beltrami agissant sur les $p$-fonctions, i.e. le laplacien agissant ligne à ligne. Reste à spécifier ce que nous entendons par $\mathcal{U}^{\frac{n+2}{n-2}}$. Deux choix naturels sont possibles. Le premier, étudié dans [39], consiste à poser, en coordonnées,

$$
\left(\mathcal{U}^{\frac{n+2}{n-2}}\right)_{i}:=\left|u_{i}\right|^{\frac{4}{n-2}} u_{i}
$$

et cela correspond à un système faiblement couplé. En effet, dans ce cas, le couplage entre les lignes n'a lieu que dans le potentiel, par l'intermédiaire de la matrice de couplage $\mathcal{A}$. C'est le système le plus simple à étudier et nous ne décrirons pas ici les résultats obtenus dans ce cadre dans [39]. Le deuxième, étudié dans [41], est beaucoup plus intéressant et correspond à un système fortement couplé. Dans ce deuxième cas,

$$
\mathcal{U}^{\frac{n+2}{n-2}}:=|\mathcal{U}|^{\frac{4}{n-2}} \mathcal{U}
$$

où $|$.$| est la norme l^{2}$ dans $\mathbb{R}^{p}$. Ici, le couplage entre les lignes a lieu à la fois dans le potentiel, par l'intermédiaire de la matrice de couplage $\mathcal{A}$, et dans la non-linéarité. A partir de maintenant, c'est ce système fortement couplé que nous considérerons. Il peut donc s'écrire

$$
\Delta_{g} \mathcal{U}+\mathcal{A} \mathcal{U}=|\mathcal{U}|^{\frac{4}{n-2}} \mathcal{U}
$$

ou, en coordonnées,

$$
\Delta_{g} u_{i}+\sum_{j=1}^{p} \mathcal{A}_{i j} u_{j}=\left(\sum_{j=1}^{p} u_{j}^{2}\right)^{\frac{2}{n-2}} u_{i}
$$

pour $i=1, \ldots, p$ où les $\left(u_{i}\right)$ sont les coordonnées de $\mathcal{U}$ et les $\left(\mathcal{A}_{i j}\right)$ les entrées de la matrice de couplage $\mathcal{A}$. Nous nous intéresserons dans la suite

11. Une référence assez générale sur ces systèmes et leur rôle en physique est [1]. 
à des solutions positives ou nulles de ce système où, par $\mathcal{U} \geq 0$, nous entendons $u_{i} \geq 0$ pour tout $i=1, \ldots, p$.

Nous mesurerons la stabilité de ce système par rapport aux perturbations de la matrice de couplage $\mathcal{A}$. Ainsi, nous dirons que le système (4.8) est $\Lambda$-stable si pour tout $\varepsilon>0$, il existe $\eta>0$ tel que

$$
\left\|\mathcal{A}^{\prime}-\mathcal{A}\right\|_{C^{1}} \leq \eta \Rightarrow d_{C^{2}}^{\hookrightarrow}\left(\mathcal{S}_{\mathcal{A}^{\prime}}^{\Lambda+\varepsilon}, \mathcal{S}_{\mathcal{A}}^{\Lambda}\right) \leq \varepsilon
$$

où

$$
d_{C^{2}}^{\hookrightarrow}(X, Y)=\sup _{\mathcal{U} \in X} \inf _{\mathcal{V} \in Y}\|\mathcal{U}-\mathcal{V}\|_{C^{2}}
$$

les normes $C^{k}$ pour des $p$-fonctions ou des fonctions de matrices étant les sommes des normes $C^{k}$ des coordonnées et où

$$
\mathcal{S}_{\mathcal{A}}^{\Lambda}=\{\mathcal{U} \text { solution de }(4.8) \text { telle que } \mathcal{E}(\mathcal{U}) \leq \Lambda\}
$$

l'énergie $\mathcal{E}(\mathcal{U})$ d'une $p$-fonction étant définie par

$$
\mathcal{E}(\mathcal{U})=\int_{M}|\mathcal{U}|^{\frac{2 n}{n-2}} d v_{g} .
$$

Dire que le système est $\Lambda$-stable revient donc à dire qu'une petite perturbation de la matrice de couplage ne crée pas de solutions d'énergie plus petite que $\Lambda$ loin de toute solution du système initial.

Nous définirons comme dans le chapitre 2 la forte stabilité comme étant la $\Lambda$-stabilité plus la $\Lambda$-compacité pour tout $\Lambda>0$. Nous nous sommes attachés dans [41] à trouver des conditions sur la matrice de couplage $\mathcal{A}$ pour que le système soit fortement stable. Avant d'énoncer le résultat, nous avons besoin d'introduire deux hypothèses sur la matrice de couplage $\mathcal{A}$. La première concerne le noyau de l'opérateur $\Delta_{g}+\mathcal{A}$ :

$$
\text { (H) } \operatorname{Ker}\left(\Delta_{g}+\mathcal{A}\right) \cap L^{2}\left(M, V e c t_{+}\left(\mathbb{R}^{p}\right)\right)=\{0\}
$$

où $V e c t_{+}\left(\mathbb{R}^{p}\right)$ est l'ensemble des vecteurs de $\mathbb{R}^{p}$ avec des composantes positives ou nulles. Pour introduire la seconde hypothèse, notons

$$
\mathcal{A}_{n}=\mathcal{A}-\frac{n-2}{4(n-1)} S_{g} I d
$$

où $S_{g}$ est la courbure scalaire de $(M, g)$ et $I d$ est la matrice identité. Pour $x \in M$, notons $I s_{\mathcal{A}_{n}(x)}$ l'espace vectoriel des vecteurs isotropes ${ }^{12}$ de $\mathcal{A}_{n}$. La deuxième hypothèse est que, pour tout $x \in M, \mathcal{A}_{n}(x)$ ne doit pas posséder de sous-espaces stables avec une base orthonormée composée de vecteurs isotropes à composantes positives ou nulles. En d'autres termes, nous supposerons que

\footnotetext{
12. Un vecteur isotrope d'une matrice $B$ est un vecteur $X$ tel que $\langle B X, X\rangle=0$ où $\langle.,$.$\rangle est le produit scalaire euclidien de \mathbb{R}^{p}$.
} 
(H') Pour tout $x \in M$ et tout $k \in\{1, \ldots, p\}$, il n'existe pas de famille orthonormale $\left(e_{1}, \ldots, e_{k}\right)$ de vecteurs dans $I_{\mathcal{A}_{n}(x)} \cap V$ ect $+\left(\mathbb{R}^{p}\right)$ telle que l'espace vectoriel engendré par cette famille soit stable par $\mathcal{A}_{n}$.

Le cas $k=1$ dans l'hypothèse (H') revient à dire que le noyau de $\mathcal{A}_{n}(x)$ ne possède pas de vecteur non-trivial à composantes positives ou nulles. L'hypothèse (H') est satisfaite dans plusieurs situations simples. C'est le cas par exemple si $\mathcal{A}_{n}(x)$ ne possède pas de vecteurs isotropes, ce qui est assuré par exemple par une hypothèse du type $\mathcal{A}_{n}(x)$ définie positive (ou négative) pour tout $x \in M$. Dans le cas $p=2$, (H') est satisfaite dès lors que $\operatorname{det} \mathcal{A}_{n}(x)>0$ pour tout $x \in M$.

Autant l'hypothèse $(\mathrm{H})$ est analytique de nature, l'hypothèse (H') est elle algébrique. Elle est d'ailleurs reliée à la structure conforme sous-jacente à notre système. Mais, par rapport à la situation pour les équations de type Yamabe, elle prend en compte l'interaction entre les lignes ${ }^{13}$.

Le résultat est alors le suivant :

Théorème 4.2. [Druet-Hebey, [41]] Soit $(M, g)$ une variété riemannienne compacte de dimension $n \geq 4$, soit $p \geq 1$ un entier. Pour toute fonction $\mathcal{A}: M \mapsto M_{p}^{s}(\mathbb{R})$ de classe $C^{1}$ satisfaisant les hypothèses $(H)$ et $\left(H^{\prime}\right)$, le système (4.8) est fortement $\Lambda$-stable pour tout $\Lambda>0$ si $n \neq 6$. De plus, il existe des exemples de variétés de dimension 6 et de fonctions $\mathcal{A}$ de classe $C^{1}$ vérifiant $(H)$ et $\left(H^{\prime}\right)$ pour lesquelles le système (4.8) n'est pas fortement $\Lambda$-stable dès que $\Lambda$ est suffisamment grand.

Pour démontrer ce théorème, il faut : une théorie ponctuelle des défauts de compacité pour ces systèmes couplés, une estimation des rayons d'influence des bulles (cf. chapitre 3 pour le cas scalaire). Si la preuve de ce théorème suit les schémas de preuve de [35] et [42], au prix d'un sérieux remaniement tout de même, elle a également bénéficié de quelques travaux préliminaires sur ces systèmes, en particulier [53, 54].

Nous n'allons pas reprendre le schéma de preuve d'un tel résultat mais juste en pointer les difficultés principales. La première, de taille, est l'absence totale de principe du maximum pour ces systèmes. Par exemple, une composante de $\mathcal{U}$ peut parfaitement s'annuler quelque part sans pour autant être identiquement nulle. Le principe de comparaison étant essentiel dans la preuve des estimées ponctuelles, cela crée bien entendu des complications. Nous sommes en fait sauvés par le fait que la somme des composantes de $\mathcal{U}$ vérifie elle un principe du maximum, ce qui permet de faire passer un certain nombre d'estimées. Reste à travailler pour passer d'estimées sur les composantes prises dans leur ensemble à des estimées sur

13. L'hypothèse pour $k=1$ serait l'analogue de l'hypothèse du théorème 2.1. Mais cette hypothèse pour $k \geq 2$ est liée au fait que nous regardons ici un système d'EDP elliptiques. 
les composantes individuelles. Cette théorie ponctuelle permet de décrire une suite de solutions du système qui explose en termes de bulles standard modelées sur les solutions du système $\Delta \mathcal{U}=|\mathcal{U}|^{\frac{4}{n-2}} \mathcal{U}$ dans l'espace entier $\mathbb{R}^{n}$. Les solutions de ce système sont classifiées dans [41] et [43].

Une deuxième difficulté, certes banale à énoncer mais bien entendu fondamentale, est que les composantes de $\mathcal{U}$ interagissent. Même si seule une composante de $\mathcal{U}$ développe un point de concentration quelque part, il est clair que les autres lignes en seront affectées puisque l'équation qui les régit va devenir singulière. $\mathrm{Si}$, de plus, plusieurs composantes développent des points de concentration, à des échelles différentes, plus ou moins proches les uns des autres, il est clair que les interactions risquent d'être difficiles à comprendre dans le détail, chose nécessaire pour obtenir un résultat de stabilité digne de ce nom. C'est tout le travail sur les rayons d'influence qui s'en trouve perturbé et qui demande à être repris en grande partie.

Pour ce qui est de la forte stabilité globale pour ces systèmes, nous avons obtenu dans [43] un résultat qui est ici le parfait analogue, dans son énoncé, du théorème 2.2 :

Théorème 4.3 (Druet-Hebey-Vétois, [43]). Soit $(M, g)$ une variété riemannienne compacte de dimension $n \geq 3$, soit $p \geq 1$ un entier. Soit $\mathcal{A}: M \mapsto M_{p}^{s}(\mathbb{R})$ de classe $C^{1}$ telle que

$$
A(x)<\frac{n-2}{4(n-1)} S_{g}(x) I d_{p}
$$

au sens des formes bilinéaires pour tout $x \in M$. En dimension 3 , supposons de plus que $\Delta+A$ est coercif et que $-A$ est coopératif ${ }^{14}$. Alors le système (4.8) est globalement fortement stable.

\subsection{Stabilité de l'obstruction de Pohožaev}

Si l'équation avec croissance de Sobolev critique doit sa gloire en grande partie au problème de Yamabe et à l'analyse géométrique, si nombre d'idées nouvelles dans l'analyse de ces EDP elliptiques avec défaut de compacité sont issues de ce domaine, il ne faut pas oublier que, dès les années 1960, et parallèlement ensuite, les mêmes équations ont été étudiées dans l'espace euclidien. A partir des années 1980, ce domaine, sous l'impulsion du travail de Brézis et Nirenberg [19], a connu une extension fulgurante.

Nous allons revenir dans cette section sur le phénomène des petites dimensions, déjà mis en évidence par les travaux d'Aubin [6] en 1976, mais mis sur le devant de la scène par Brézis-Nirenberg [19] et Schoen [87].

14. Ceci signifie que $A_{i j} \leq 0$ pour tous $i \neq j$. 
La rencontre entre les deux domaines sus-cités s'est sans doute faite chez Trudinger qui découvre l'erreur de Yamabe après avoir pris connaissance du travail de Pohožaev. Résoudre l'équation

$$
\Delta u+h u=u^{q}
$$

dans un ouvert $\Omega$ avec condition de Dirichlet au bord pour $h$ une fonction régulière et bornée sur $\Omega$ n'est pas difficile tant que $q<\frac{n+2}{n-2}$. En effet, dans ce cas, il suffit par exemple de résoudre le problème de minimisation

$$
\mu=\inf _{u \in \mathcal{H}_{q+1}} \int_{\Omega}\left(|\nabla u|^{2}+h u^{2}\right) d x
$$

où $\mathcal{H}_{q+1}$ est l'ensemble des fonctions dans $H_{0}^{1}(\Omega)$, l'espace des fonctions nulles au bord dont le gradient est dans $L^{2}$, normalisées telles que $\int_{\Omega}|u|^{q+1} d x=1$. Or ce problème de minimisation est simple à résoudre car l'injection de $H_{0}^{1}(\Omega)$ dans $L^{q+1}(\Omega)$ est compacte tant que $q<\frac{n+2}{n-2}$. Une condition suffisante pour le résoudre est tout de même que la première valeur propre de l'opérateur $\Delta+h$ soit strictement positive. Mais c'est également une condition nécessaire pour avoir une solution de strictement positive de l'équation ci-dessus (cf. note page 21 ). Il suffit ensuite de remarquer que le minimum peut être pris positif ou nul et d'utiliser le principe du maximum pour trouver une solution strictement positive à l'équation ci-dessus. Pour $q=\frac{n+2}{n-2}$, tout se corse. En 1965, Pohožaev, dans [83], démontre que l'équation ci-dessus n'admet pas de solution si $h \equiv \lambda \geq 0$, $q \geq \frac{n+2}{n-2}$ et l'ouvert $\Omega$ est étoilé. Ce résultat est basé sur l'identité de Pohožaev. Nous reviendrons sur cette identité un peu plus loin, page 73 . Ainsi, résoudre cette équation dès que $q=\frac{n+2}{n-2}$ ne peut être simple. Ce problème a connu un certain nombre de développements avant les années 80, essentiellement dans le cadre des problèmes de Yamabe et de courbure scalaire prescrite. Les contributions importantes sont celles de Trudinger [97], Aubin [6], Kazdan-Warner [62, 63]. Citons également celle de Schoen [87] au début des années 80. Dans le monde euclidien, une contribution importante, qui contribua à la publicisation de ces questions, fut celle de Brézis et Nirenberg [19].

Dans [19], Brézis et Nirenberg s'intéressent à l'existence de solutions minimisantes (donc positives) de l'équation

$$
\Delta u+\lambda u=u^{\frac{n+2}{n-2}} \text { dans } \Omega, u=0 \text { sur } \partial \Omega
$$

où $\Omega$ est un ouvert lisse borné de $\mathbb{R}^{n}$. Une solution est dite minimisante si elle atteint le minimum de la fonctionnelle

$$
J(u)=\int_{\Omega}\left(|\nabla u|^{2}+\lambda u^{2}\right) d x
$$


sur l'ensemble $\mathcal{H}$ des fonctions de $H_{0}^{1}(\Omega)$ vérifiant $\int_{\Omega}|u|^{\frac{2 n}{n-2}} d x=1$. Notons

$$
\mu=\inf _{u \in \mathcal{H}} J(u)
$$

Il est facile de voir grâce à un calcul de fonctions-tests dû à Aubin [7] que

$$
\mu \leq K(n, 2)^{-2}
$$

où $K(n, 2)$ est la meilleure constante dans l'inégalité de Sobolev euclidienne. Il est également bien connu depuis [6] et même [97] que l'infimum ci-dessus est atteint dès que $\mu<K(n, 2)^{-2}$ si $\lambda>-\lambda_{1}(\Omega)$, la première valeur propre du laplacien sur $\Omega$ avec condition de Dirichlet au bord. En dimensions $n \geq 4$, en utilisant les fonctions-tests d'Aubin [6], Brezis et Nirenberg démontrent que $\mu<K(n, 2)^{-2}$ si et seulement si $\lambda<0$. De plus, une solution minimisante n'existe que si $-\lambda_{1}(\Omega)<\lambda<0$. En effet, son existence pour $\lambda \geq 0$ donnerait une fonction extrémale pour l'inégalité de Sobolev euclidienne sur tout l'espace (en l'étendant par 0 en-dehors de $\Omega)$; or celles-ci sont connues et ne sont pas à support compact. Ainsi, en dimensions $n \geq 4$, l'existence d'une solution minimisante est équivalente au fait que $\mu<K(n, 2)^{-2}$, lui-même équivalent à $\lambda<0$. Pour ce qui est de l'existence de solutions non-minimisantes, le résultat de Pohožaev [83] nous dit que, si l'ouvert $\Omega$ est étoilé, il ne peut pas exister de solutions dès que $\lambda>0$. Ceci règle intégralement la question de l'existence de solutions au problème ci-dessus dans le cas étoilé. Si $\Omega$ est un ouvert non étoilé, la situation devient plus complexe, en ce qui concerne les solutions nonminimisantes. Il peut en exister pour $\lambda \geq 0$ : c'est le cas sur les anneaux, cf. [62], ou sur des ouverts qui y ressemblent, cf. [29], et plus généralement sur tous les domaines ayant une topologie non triviale, cf. [8].

Revenons au problème des solutions minimisantes. Il n'est pas difficile d'étendre le résultat ci-dessus à l'équation

$$
\Delta u+h u=u^{\frac{n+2}{n-2}} \text { dans } \Omega, u=0 \operatorname{sur} \partial \Omega
$$

où $\Omega$ est un ouvert lisse borné de $\mathbb{R}^{n}$ et $h \in C^{\infty}(\Omega) \cap L^{\infty}(\Omega)$. Remarquons que l'existence d'une solution à ce problème entraîne la coercivité de l'opérateur $\Delta_{g}+h$, i.e. la positivité de la première valeur propre de cet opérateur sur $\Omega$ avec condition de Dirichlet au bord. Posons

$$
\mu_{h}=\inf _{u \in \mathcal{H}} \int_{\Omega}\left(|\nabla u|^{2}+h u^{2}\right) d x
$$

Encore une fois, il est clair que $\mu_{h} \leq K(n, 2)^{-2}$ et Brezis et Nirenberg [19] démontrent donc que les trois assertions suivantes sont équivalentes en dimensions $n \geq 4$ : 
(a) Il existe $x \in \Omega$ tel que $h(x)<0$.

(b) $\mu_{h}<K(n, 2)^{-2}$.

(c) $\mu_{h}$ est atteint par une fonction strictement positive solution minimisante de (4.9).

Mais le plus intéressant du travail de Brezis et Nirenberg reste la mise en lumière d'un phénomène dimensionnel. En dimension 3, la situation change drastiquement. Ils démontrèrent qu'en dimension 3 , il existe $\lambda^{\star}(\Omega) \in$ $\left(-\lambda_{1}(\Omega), 0\right)$ tel que les propositions suivantes soient vraies :

- $\mu_{\lambda}=K(3,2)^{-1}$ pour $\lambda \geq \lambda^{\star}(\Omega)$.

- $\mu_{\lambda}<K(3,2)^{-1}$ pour $\lambda<\lambda^{\star}(\Omega)$.

- $\mu_{\lambda}$ n'est pas atteint pour $\lambda>\lambda^{\star}(\Omega)$.

Cela pose évidemment la question de la caractérisation de ce $\lambda^{\star}(\Omega)$ et également de l'existence d'une solution minimisante pour $\lambda=\lambda^{\star}(\Omega)$. Dans le cas de la boule unité $B$ de $\mathbb{R}^{3}$, Brezis et Nirenberg ont démontré que $\lambda^{\star}(B)=-\frac{1}{4} \lambda_{1}(B)$ et que $\mu_{\lambda^{\star}(B)}$ n'est pas atteint.

Dans [34], nous avions répondu à ces deux questions dans le cadre plus général de l'équation (4.9). Sur $\Omega$ un ouvert borné lisse de $\mathbb{R}^{3}$, étant donnée une fonction $h \in C^{\infty}(\Omega) \cap L^{\infty}(\Omega)$ telle que l'opérateur $\Delta+h$ soit coercif, nous noterons $\mathcal{G}_{h}: \Omega \times \Omega \mapsto \mathbb{R}$ sa fonction de Green avec condition de Dirichlet au bord. Cette fonction est symétrique, régulière en-dehors de la diagonale, positive, et vérifie au sens des distributions

$$
\Delta_{y} \mathcal{G}_{h}(x, y)+h(y) \mathcal{G}_{h}(x, y)=\delta_{x}
$$

pour tout $x \in \Omega$ et $\mathcal{G}_{h}(x, y)=0$ si $x \in \Omega$ et $y \in \partial \Omega$. Cette fonction de Green admet le développement limité

$$
\mathcal{G}_{h}(x, y)=\frac{1}{\omega_{2}|x-y|}+M_{h}(x)+o(1)
$$

pour $y$ proche de $x$. La constante $M_{h}(x)$ est appelée la masse de la fonction de Green au point $x$. Nous avions démontré dans [34] que les trois assertions suivantes sont équivalentes :

(a) Il existe $x \in \Omega$ tel que $M_{h}(x)>0$.

(b) $\mu_{h}<K(3,2)^{-1}$.

(c) $\mu_{h}$ est atteint par une fonction strictement positive solution minimisante de (4.9).

Ainsi la question de l'existence de solutions minimisantes est parfaitement bien comprise en dimension 3 également.

Dans [44], nous avons tenté avec Paul Laurain de creuser un peu plus ce phénomène de petites dimensions en étudiant la stabilité de l'obstruction 
de Pohožaev en dimension 3. Considérons une solution de l'équation (4.9). Multiplier cette équation par $x^{i} \partial_{i} u$ et intégrer par parties mène à la célèbre identité de Pohožaev :

$$
\int_{\Omega}\left(h+\frac{1}{2} x^{i} \partial_{i} h\right) u^{2} d x=-\frac{1}{2} \int_{\partial \Omega}(x, \nu)\left(\partial_{\nu} u\right)^{2} d \sigma .
$$

Cette identité ne sort pas de nulle part. L'idée de départ est que l'équation $\Delta u=u^{\frac{n+2}{n-2}}$ est invariante par le changement d'échelle $u_{\lambda}=\lambda^{\frac{n-2}{2}} u(\lambda x)$. Ceci signifie que tous les $u_{\lambda}$ vérifient l'équation $\Delta u_{\lambda}=u_{\lambda}^{\frac{n+2}{n-2}}$. Différentier cette équation en $\lambda=1$ nous donne une solution de l'équation linéarisée : la fonction $v=x^{k} \partial_{k} u+\frac{n-2}{2} u$ vérifie $\Delta v=\frac{n+2}{n-2} u^{\frac{4}{n-2}} v$. En fait, l'équation linéarisée admet d'autres solutions ${ }^{15}$ liées à l'invariance de l'équation par toute translation en espace. Ces autres solutions mèneraient à d'autres identités de Pohožaev, toutefois moins intéressantes pour notre propos. Voyons en quoi cette remarque mène à l'identité de Pohožaev. Etant donnée une solution $u$ de l'équation (4.9), la fonction $v=x^{k} \partial_{k} u+\frac{n-2}{2} u$ sera presque solution de l'équation linéarisée, le presque étant dû à la présence d'un bord et à celle d'un potentiel $h$. Tester l'équation (4.9) contre la fonction $v$ devrait donc relier quelques quantités contenant des termes de bord et la fonction $h$. En effet, $v$ vérifie l'équation

$$
\Delta v+h v=\frac{n+2}{n-2} u^{\frac{4}{n-2} v}-\left(x^{k} \partial_{k} h+2 h\right) u .
$$

Multiplions l'équation (4.9) par $v$ et intégrons par parties. En utilisant le fait que

$$
\int_{\Omega} u^{\frac{n+2}{n-2}} v d x=0
$$

ce qui est fondamentalement dû à l'invariance de la norme $L^{\frac{2 n}{n-2}}$ par le changement d'échelle ci-dessus, nous obtenons

$$
\begin{aligned}
0 & =\int_{\Omega}(\Delta u+h u) v d x \\
& =-\int_{\partial \Omega} v \partial_{\nu} u d \sigma+\int_{\Omega}(\Delta v+h v) u d x \\
& =-\int_{\partial \Omega}(x, \nu)\left(\partial_{\nu} u\right)^{2} d \sigma-\int_{\Omega}\left(x^{k} \partial_{k} h+2 h\right) u^{2} d x
\end{aligned}
$$

ce qui n'est rien d'autre que (4.10).

15. Pour une étude complète de cette équation linéarisée sur l'espace entier, cf. Bianchi-Egnell [13]. 
Grâce à (4.10), l'équation (4.9) ne peut avoir de solutions non-triviales dès que $\Omega$ est étoilé par rapport à l'origine ${ }^{16}$ et que

$$
h+\frac{1}{2} x^{i} \partial_{i} h \geq 0 .
$$

C'est ce qui est appelé l'obstruction de Pohožaev.

Par contre, dès que $\Omega$ n'est plus étoilé ou que $h$ ne vérifie plus cette condition, il est possible d'obtenir des résultats d'existence dont il est illusoire d'espérer donner une liste ${ }^{17}$.

Nous allons ici nous intéresser à la stabilité de l'obstruction de Pohožaev. Partant d'un domaine étoilé $\Omega$ et d'une fonction $h$ vérifiant (4.11), nous voulons savoir si la propriété "il n'existe pas de solutions non-triviales au problème (4.9)" est stable par perturbation. Nous ne considérerons que des perturbations de la fonction $h$ mais il serait tout-à-fait plausible de faire le même travail pour des perturbations du domaine $\Omega$. Voici ce que nous entendons exactement par stabilité de l'obstruction de Pohožaev :

Définition 4.1. Soit $\Omega$ un domaine étoilé (par rapport à 0 ) de $\mathbb{R}^{n}$, soit $\left(X,\|\|_{X}\right)$ un espace de Banach de fonctions sur $\Omega$. Soit $h_{0} \in X \cap C^{1}(\Omega)$ une fonction vérifiant (4.11). Nous dirons que l'obstruction de Pohožaev est $X$-stable en $\left(h_{0}, \Omega\right)$ s'il existe $\delta\left(h_{0}, \Omega, X\right)>0$ tel que pour toute fonction $h \in X$ avec $\left\|h-h_{0}\right\|_{X} \leq \delta\left(h_{0}, \Omega, X\right)$, la seule solution $C^{2}$ de (4.9) est la solution triviale.

Nous dirons que l'obstruction de Pohožaev est $X$-stable si elle est $X$ stable en $\left(h_{0}, \Omega\right)$ pour tout domaine $\Omega$ étoilé et toute fonction $h_{0} \in X \cap$ $C^{1}(\Omega)$ vérifiant (4.11).

Evidemment, la propriété (4.11) n'est elle pas stable sous des perturbations $C^{k}$ de $h$, ce qui rend la question de la stabilité de l'obstruction de Pohožaev intéressante. En dimensions $n \geq 4$, l'obstruction de Pohožaev n'est $X$-stable pour aucun $X$ raisonnable. En effet, toute perturbation de $h_{0} \equiv 0$, qui vérifie (4.11), qui sera négative quelque part conduira à l'existence d'une solution minimisante, non-triviale, de (4.9) en dimensions $n \geq 4$ (cf. discussion ci-dessus) ${ }^{18}$. Par contre, en dimension 3 , le cas des solutions minimisantes peut laisser espérer une certaine stabilité de l'obstruction de Pohožaev. Nous avons obtenu dans [44] le résultat suivant, qui, à nos yeux, est une excellente illustration de ce phénomène dimensionnel :

\footnotetext{
16. ou par rapport à n'importe quel point à condition de modifier de manière adéquate la condition (4.11), puisque le problème est invariant par translation.

17. Ce problème a fait l'objet d'une myriade de travaux dans les 20 dernières années.

18. Nous ne disons rien sur la $X$-stabilité en un couple précis $(h, \Omega)$ en dimensions $n>4$. Il pourrait être intéressant de voir si l'obstruction de Pohožaev peut être stable en dimensions $n \geq 4$ autour de certaines fonctions $h$, dans les cas où ce n'est pas trivial, voire de caractériser ces fonctions $h$.
} 
Théorème 4.4. [Druet-Laurain, [44]] L'obstruction de Pohožaev est $C^{0, \eta}$ stable pour tout $\eta>0$ en dimension 3. En d'autres termes, étant donnés $\eta>0, \Omega$ un domaine de $\mathbb{R}^{3}$ étoilé par rapport à l'origine, et une fonction $h_{0} \in C^{1}(\Omega)$ vérifiant (4.11), il existe $\delta\left(\eta, \Omega, h_{0}\right)>0$ tel que pour toute fonction $h \in C^{0, \eta}(\Omega)$, si $\left\|h-h_{0}\right\|_{C^{0, \eta}(\Omega)} \leq \delta$, le problème (4.9) n'admet pas de solutions non triviales.

Une conséquence de ce résultat en dimension 3 est la suivante : si $\Omega$ est un domaine étoilé de $\mathbb{R}^{3}$, il existe une constante $\hat{\lambda}(\Omega)<0$ telle que l'équation (4.9) ne possède pas de solutions non-triviales avec $h \equiv \lambda$ dès lors que $\lambda>\hat{\lambda}(\Omega)$. Cette situation est clairement différente de ce qui se passe pour certains domaines non-étoilés, en particulier les domaines à trous (cf. [8]).

Dans [20], Brezis et Willem ont étudié cette question dans le cas de la boule unité pour des fonctions radiales. Notons $L_{r}^{p}(B)$ l'ensemble des fonctions radiales qui sont dans $L^{p}(B)$. Il est démontré dans [20] que l'obstruction de Pohožaev est $L_{r}^{\infty}$-stable dans la boule unité de $\mathbb{R}^{3}$ pour toute fonction $h \in L_{r}^{\infty}(B) \cap C^{1}(B)$. Dans [20], la question était explicitement posée d'une extension de ce résultat au cas non-radial. Si notre résultat fournit une réponse à cette question, le problème se révèle plus subtil que prévu dans le cas non-radial puisque l'identité de Pohožaev n'est jamais $L^{\infty}$-stable :

Théorème 4.5. [Druet-Laurain, [44]] L'obstruction de Pohožaev n'est jamais $L^{\infty}$-stable. En d'autres termes, étant donnés un domaine $\Omega$ de $\mathbb{R}^{3}$ étoilé par rapport à l'origine et une fonction $h_{0} \in L^{\infty}(\Omega) \cap C^{1}(\Omega)$ satisfaisant (4.11), pour tout $\varepsilon>0$, il existe une fonction $h_{\varepsilon} \in C^{\infty}(\Omega) \cap L^{\infty}(\Omega)$ telle que $\left\|h_{\varepsilon}-h_{0}\right\|_{L^{\infty}(\Omega)} \leq \varepsilon$ et une fonction $u_{\varepsilon}$ de classe $C^{2}$ strictement positive vérifiant

$$
\Delta u_{\varepsilon}+h_{\varepsilon} u_{\varepsilon}=u_{\varepsilon}^{5} \text { dans } \Omega
$$

et $u_{\varepsilon}=0$ sur $\partial \Omega$.

Ainsi la $L_{r}^{\infty}$-stabilité est spécifique au cas radial. En fait, le résultat n'est pas dû tant à la symétrie radiale qu'à une de ses conséquences en dimension 3 qui est qu'une suite de solutions de perturbations de l'équation (4.9) qui sont radiales ne peut développer qu'un point de concentration (en 0) si elle n'est pas compacte. De plus, grâce aux techniques d'EDP utilisées, à comparer aux techniques d'EDO de [20], nous pouvons raffiner le résultat dans le cas radial :

Théorème 4.6. [Druet-Laurain, [44]] L'obstruction de Pohožaev est $L_{r}^{p}$ stable dans la boule unité de $\mathbb{R}^{3}$ pour tout $p>3$ mais n'est pas $L_{r}^{3}$-stable.

Cet ensemble de résultats dresse un tableau complet de la stabilité de l'obstruction de Pohožaev en dimension 3. Une question extrêmement 
intéressante et difficile est de savoir si ces résultats s'étendent au cas des solutions qui changent de signe. L'obstruction de Pohožaev, elle, s'étend à ce cadre. Cette question est très subtile à cause de la variété de solutions qui changent de signe à l'équation-modèle $\Delta u=u^{5}$ dans $\mathbb{R}^{3}$. En particulier, le monde des "bulles-standard" s'agrandit considérablement dans ce cadre. Une simple question, peut-être plus abordable, est la suivante : existe-t-il des solutions qui changent de signe à l'équation (4.9) sur la boule unité de $\mathbb{R}^{3}$ pour $-\frac{1}{4} \lambda_{1}(B)<\lambda<0$ alors qu'il n'en existe pas dès que $\lambda \geq 0$ et qu'il n'en existe pas de positives dans cet intervalle (voir [17] et $[19]) ?$

Disons quelques mots rapides sur la preuve de ces résultats. Démontrer que l'obstruction de Pohožaev est stable en $h_{0}$ revient à démontrer que l'équation (4.9) est stable avec $h=h_{0}$. Considérons la démonstration du théorème 4.4. En raisonnant par l'absurde, cela revient à démontrer que l'existence d'une suite de solutions $u_{\varepsilon}$ aux équations

$$
\Delta u_{\varepsilon}+h_{\varepsilon} u_{\varepsilon}=u_{\varepsilon}^{5} \text { dans } \Omega, u_{\varepsilon}>0 \text { dans } \Omega, u_{\varepsilon}=0 \text { sur } \partial \Omega
$$

avec $h_{\varepsilon} \rightarrow h_{0}$ dans $C^{0, \eta}(\Omega)$ quand $\varepsilon \rightarrow 0$ mène à une contradiction. Or, l'équation-limite ne possédant pas de solutions, une telle suite $\left(u_{\varepsilon}\right)$ ne peut exister que si elle développe un certain nombre de points de concentration. Il faut démontrer que ces points de concentration sont en nombre fini (ce qui est classique en dimension 3 dès lors qu'ils se situent à l'intérieur de l'ouvert $\Omega$ mais qui devient délicat lorsque les points de concentration s'approchent du bord). Eliminer l'existence de points de concentration au bord pose problème car se crée un point de concentration artificiel à l'extérieur du domaine (par "symétrie orthogonale") qui a tendance à compenser l'effet des autres points de concentration et donc de rendre le recollement plus plausible. Malgré tout, cela est faisable. Le résultat est donc que la suite $\left(u_{\varepsilon}\right)$ développe un nombre fini de points de concentration, tous situés dans l'intérieur de $\Omega$. En utilisant l'identité de Pohožaev autour de ces points de concentration, nous obtenons ensuite des relations d'équilibre entre ces divers points faisant intervenir la fonction de Green de $\Delta+h_{0}$. C'est pour obtenir ces relations que la convergence dans $C^{0, \eta}$ est absolument nécessaire. S'il n'y a qu'un point, une convergence dans $L^{p}, p>3$, suffit. Ensuite, il faut démontrer que ces relations d'équilibre ne peuvent pas être satisfaites, ce qui repose sur une jolie identité de type Pohožaev sur les fonctions de Green.

La construction des exemples menant à l'instabilité est quant à elle extrêmement délicate puisque le résultat est précis. La suite de fonctions $\left(h_{\varepsilon}\right)$ doit converger dans $L^{\infty}$ mais dans aucun $C^{0, \eta}$. Il faut remarquer que notre construction est explicite. Etant donnée la fonction $h_{0}$, nous pouvons donner une suite $\left(h_{\varepsilon}\right)$ explicite, en termes de fonction de Green de l'opérateur $\Delta+h_{0}$, qui converge dans $L^{\infty}$ vers $h_{0}$ et pour laquelle 
l'équation (4.9) a toujours des solutions. En fait, nous démontrons que, sur tout ouvert borné régulier $\Omega$ de $\mathbb{R}^{3}$, l'ensemble des fonctions $h$ pour lesquelles l'équation (4.9) admet une solution non triviale est $L^{\infty}$-dense dans l'espace des fonctions pour lesquelles l'opérateur $\Delta+h$ est coercif.

\subsection{Des équations d'ordre 4}

Nous allons donner une première illustration, particulièrement simple, de la puissance de la méthode ponctuelle quand il s'agit de quantifier les niveaux d'énergie auxquels des défauts de compacité peuvent se produire. Nous allons nous intéresser à des équations d'ordre 4 issues de la géométrie conforme (au sens où les équations d'ordre 2 du chapitre 2 l'étaient).

Soit $(M, g)$ une variété riemannienne compacte de dimension 4. Considérons sur celle-ci l'équation d'ordre 4 avec non-linéarité exponentielle :

$$
\Delta_{g}^{2} u-d i v_{g}(A d u)+b=f e^{u}
$$

où $b$ et $f$ sont deux fonctions régulières sur $M$ et $A$ est un champ de $(2,0)$-tenseurs symétriques. Cette équation est modelée sur l'équation de $Q$-courbure, équation de prescription de la $Q$-courbure dans une classe conforme qui est en dimension 4 l'analogue de l'équation de prescription de la courbure scalaire ${ }^{19}$ en dimension 2. La $Q$-courbure a fait l'objet de nombreux travaux ces dernières années. Nous renvoyons sur ce sujet à l'article de survol [23] et aux références qui y sont contenues (voir aussi $[76])$.

Considérons maintenant une suite $\left(u_{\alpha}\right)$ de solutions de

$$
\Delta_{g}^{2} u_{\alpha}-\operatorname{div}_{g}\left(A_{\alpha} d u_{\alpha}\right)+b_{\alpha}=f_{\alpha} e^{u_{\alpha}}
$$

où les suites $\left(b_{\alpha}\right),\left(f_{\alpha}\right)$ et $\left(A_{\alpha}\right)$ convergent vers des objets de même nature $b_{0}, f_{0}$ et $A_{0}$, en topologie $C^{2}$ - pour fixer les idées mais ce n'est pas optimal. Supposons que la suite $\left(u_{\alpha}\right)$ explose lorsque $\alpha \rightarrow+\infty$, i.e. $\sup _{M} u_{\alpha} \rightarrow$ $+\infty$ quand $\alpha \rightarrow+\infty$. Le théorème ci-dessous donne alors un résultat de quantification d'énergie pour les niveaux d'explosion mais également une asymptotique relativement précise de la suite $\left(u_{\alpha}\right)$ et des informations sur la localisation éventuelle des points de concentration. Avant d'énoncer le résultat, quelques notations sont nécessaires. Nous noterons $L_{0}=\Delta_{g}^{2}-$ $\operatorname{div}_{g}\left(A_{0} d u\right)$. Nous supposerons dans la suite que son noyau ne contient que les constantes. Alors sa fonction de Green $G$ est bien définie à une

19. Celle-ci s'écrit, en dimension $2, \Delta_{g} u+S_{g}=f e^{u}$. 
constante près ${ }^{20}$ et peut s'écrire sous la forme

$$
G(x, y)=\frac{1}{8 \pi^{2}} \ln \frac{1}{d_{g}(x, y)}+\beta(x, y)
$$

pour $(x, y) \in M^{2}, x \neq y$. Ici, la fonction $\beta$ est dans $C^{1}(M \times M)$. C'est la partie régulière de la fonction de Green $G$. Posons également

$$
\varphi(x)=\int_{M} G(x, y) b_{0}(y) d v_{g} .
$$

Théorème 4.7. [Druet-Robert, [45] et Malchiodi [75]] Soit $(M, g)$ une variété riemannienne de dimension 4 . Soit $\left(u_{\alpha}\right)$ une suite de solutions de (4.12) qui explose. Supposons que le noyau de $L_{0}$ ne contienne que les fonctions constantes et que la limite $f_{0}$ soit strictement positive. Alors, à extraction près d'une sous-suite,

(a) (quantification) Il existe $N \in \mathbb{N}^{\star}$ tel que

$$
\int_{M} b_{0} d v_{g}=64 \pi^{2} N
$$

(b) (asymptotique) Il existe $\left\{x_{i}\right\}_{i=1, \ldots, N}$ tel que

$$
u_{\alpha}-\frac{1}{V o l_{g}(M)} \int_{M} u_{\alpha} d v_{g} \rightarrow 64 \pi^{2} \sum_{i=1}^{N} G\left(x_{i}, .\right)-\varphi
$$

dans $C_{\text {loc }}^{2}\left(M \backslash\left\{x_{i}\right\}_{i=1, \ldots, N}\right)$ quand $\alpha \rightarrow+\infty$.

(c) (localisation) Enfin,

$$
64 \pi^{2} \nabla_{y} \beta\left(x_{i}, x_{i}\right)+64 \pi^{2} \sum_{j \neq i} \nabla_{x} G\left(x_{i}, x_{j}\right)-\nabla \varphi\left(x_{i}\right)=-\frac{\nabla f_{0}\left(x_{i}\right)}{f_{0}\left(x_{i}\right)}
$$

pour tout $1 \leq i \leq N$.

Le point (a) correspond bien à la quantification annoncée. Celle-ci a lieu sur l'intégrale de $b_{0}$ qui correspond également à $\lim _{\alpha \rightarrow+\infty} \int_{M} f_{\alpha} e^{u_{\alpha}} d v_{g}$, ce qui joue le rôle d'énergie ici. Le point (b) donne un comportement asymptotique de la suite $\left(u_{\alpha}\right)$ loin des points de concentration qui sont d'ailleurs isolés (il n'y a pas de phénomène de bulles sur bulles pour des non-linéarités

20. Que nous fixons pour la suite, par exemple en demandant que le minimum de la fonction de Green soit 1. Mais ce choix est arbitraire et le lecteur pourra s'assurer que les résultats du théorème 4.7 sont invariants par ajout d'une constante à cette fonction de Green. 
exponentielles en dimension critique). Le comportement de la suite tout près des points de concentration est régi par un profil standard. Il resterait à obtenir un comportement asymptotique dans la zone intermédiaire. Ceci est partiellement fait - mais de manière non optimale - au cours de la preuve de [45]. Le point (c) est une contrainte sur la localisation de ces différents points de concentration. Il convient de remarquer que seul ce dernier point dépend de la fonction $f_{0}$ (hormis le fait qu'elle soit strictement positive).

Le point (a) donne une condition simple sur l'intégrale de $b_{0}$ pour que l'ensemble des solutions de l'équation soit borné dans $C^{2}(M)$. Il suffit que $\frac{1}{64 \pi^{2}} \int_{M} b_{0} d v_{g}$ ne soit pas un entier. En particulier, des résultats de compacité pour l'équation de $Q$-courbure prescrite en découlent. Ce point précis du théorème a été démontré indépendamment par Malchiodi [75] et est utilisé de manière cruciale en combinaison avec des travaux de Ding-Jost-Li-Wang [32] dans [33] pour obtenir des résultats d'existence de $Q$-courbure constante dans une classe conforme donnée (moyennant que l'intégrale de cette courbure évite un ensemble dénombrable de valeurs). Les points (b) et (c) sont une première étape dans l'analyse des défauts de compacité lorsque $\int_{M} b_{\alpha} d v_{g}$ traverse une des valeurs interdites par le point (a) et laissent un espoir pour compter ${ }^{21}$ précisément le nombre de solutions de l'équation pour toute fonction $b_{0}$. Sur ce sujet, les travaux de Chen-Lin [24], voir aussi [72], en dimension 2 sont instructifs et ceux de [73] en dimension 4 constituent une étape supplémentaire.

Indiquons en quelques étapes comment se démontre un tel résultat. Tout d'abord, prenons un point de maximum $x_{\alpha}$ de la fonction $u_{\alpha}$, i.e. $u_{\alpha}\left(x_{\alpha}\right)=\max _{M} u_{\alpha}$, qui, par hypothèse, tend vers $+\infty$ quand $\alpha \rightarrow+\infty$. Le changement d'échelle adapté - le réglage du microscope - à ce genre d'équations est bien connu depuis les travaux sur des non-linéarités exponentielles avec le laplacien en dimension 2 : en posant

$$
v_{\alpha}(x)=u_{\alpha}\left(\exp _{x_{\alpha}}\left(\mu_{\alpha} x\right)\right)-u_{\alpha}\left(x_{\alpha}\right)
$$

pour $x \in B_{0}\left(\delta \mu_{\alpha}^{-1}\right), 0<\delta<\frac{1}{2} i_{g}(M)$ fixé avec $\left(\mu_{\alpha}\right)$ une suite de réels strictement positifs tendant vers 0 lorsque $\alpha \rightarrow+\infty$ définie par

$$
f_{\alpha}\left(x_{\alpha}\right) \mu_{\alpha}^{4} e^{u_{\alpha}\left(x_{\alpha}\right)}=1,
$$

il est possible de montrer que

$$
v_{\alpha} \rightarrow V \text { dans } C_{\text {loc }}^{2}\left(\mathbb{R}^{4}\right) \text { quand } \alpha \rightarrow+\infty
$$

où $V$ vérifie l'équation

$$
\Delta_{\xi}^{2} V=e^{V}
$$

21. au sens de la théorie du degré. 
dans $\mathbb{R}^{4}$ et $V \leq V(0)=0$. De plus, $e^{V} \in L^{1}\left(\mathbb{R}^{4}\right)$. Ceci vient du fait que $\left(\int_{M} f_{\alpha} e^{u_{\alpha}} d v_{g}\right)$ est uniformément bornée ${ }^{22}$. A titre de remarque, cette quantité jouant le rôle d'énergie pour notre équation, il vient gratuitement que la suite $\left(u_{\alpha}\right)$ est d'énergie uniformément bornée. Malheureusement, ceci ne suffit pas tout-à-fait pour classifier exactement l'ensemble des solutions de l'équation-limite ci-dessus. En effet, Lin [74] a démontré que, moyennant ces informations sur $V$, soit

$$
V(x)=-4 \ln \left(1+\frac{|x|^{2}}{8 \sqrt{6}}\right),
$$

soit il existe $a>0$ tel que $\Delta_{\xi} V \geq a$ sur $\mathbb{R}^{4}$. Ce défaut de classification ${ }^{23}$ est essentiellement dû à la présence de fonctions dans le noyau de $\Delta_{\xi}^{2}$ qui sont majorées sur $\mathbb{R}^{4}$ et qui ne sont pas constantes ${ }^{24}$. Tout le problème est donc d'éliminer la deuxième possibilité. Ceci peut se faire en utilisant l'équation vérifiée par $u_{\alpha}$ et plus précisément en écrivant grâce à la formule de représentation de Green que

$$
\begin{aligned}
& \mu_{\alpha}^{-2} \int_{B_{x_{\alpha}}\left(R \mu_{\alpha}\right)}\left|\Delta_{g} u_{\alpha}\right| d v_{g} \\
& \leq C \mu_{\alpha}^{-2} \int_{x \in B_{x_{\alpha}}\left(R \mu_{\alpha}\right)} \int_{y \in M}\left|\Delta_{g} G_{\alpha}(x, y)\right|\left(e^{u_{\alpha}}(y)+1\right) d v_{g}(y) d v_{g}(x) \\
& \leq C \int_{y \in M}\left(e^{u_{\alpha}}(y)+1\right)\left(\mu_{\alpha}^{-2} \int_{x \in B_{x_{\alpha}}\left(R \mu_{\alpha}\right)} d_{g}(x, y)^{-2} d v_{g}(x)\right) d v_{g}(y) \\
& \leq C R^{2}
\end{aligned}
$$

où $C$ est une constante indépendante de $R$ et $\alpha$ changeant d'une ligne à l'autre, $G_{\alpha}$ est la fonction de Green de l'opérateur $\Delta_{g}^{2}-\operatorname{div}_{g}\left(A_{\alpha} d.\right)$, opérateur qui a un noyau réduit aux constantes pour $\alpha$ grand puisque cette propriété a lieu à la limite. Ici, il faut utiliser quelques estimées standard sur la fonction de Green. Un passage à la limite lorsque $\alpha \rightarrow+\infty$ donne alors

$$
\int_{B_{0}(R)}\left|\Delta_{\xi} V\right| d x \leq C R^{2}
$$

pour une constante $C$ indépendante de $R$. Ceci exclut clairement la deuxième possibilité et nous donne la convergence de $v_{\alpha}$ vers la solution de l'équation-limite désirée. Ainsi, l'origine de la solution $V$ nous permet tout

22. ce qui suit d'une simple intégration de l'équation satisfaite par $u_{\alpha}$.

23. à comparer avec la classification des solutions de l'équation analogue en dimension 2 , cf. [25].

24. Ceci est à comparer avec le théorème de Liouville qui assure que toute fonction harmonique majorée sur tout l'espace est constante. 
de même de la caractériser ${ }^{25}$. Il faut remarquer que le point de concentration ainsi construit transporte un quantum d'énergie puisque

$$
\lim _{R \rightarrow+\infty} \lim _{\alpha \rightarrow+\infty} \int_{B_{x_{\alpha}}\left(R \mu_{\alpha}\right)} f_{\alpha} e^{u_{\alpha}} d v_{g}=\int_{\mathbb{R}^{4}} e^{V} d x=64 \pi^{2} .
$$

Pour trouver les autres points de concentration, il faut concocter une estimée ponctuelle invariante par le changement d'échelle ci-dessus. La quantité

$$
\Phi_{\alpha}(x)=d_{g}\left(x_{\alpha}, x\right)^{4} e^{u_{\alpha}(x)}
$$

a cette propriété. En effet,

$$
\Phi_{\alpha}\left(\exp _{x_{\alpha}}\left(\mu_{\alpha} x\right)\right)=|x|^{4} f_{\alpha}\left(x_{\alpha}\right)^{-1} e^{v_{\alpha}(x)}
$$

et une borne sur $\Phi_{\alpha}$ est équivalente à une borne sur la quantité analogue construite à partir de $v_{\alpha}$. En reprenant le schéma de preuve de la section 3.1.3, il est ainsi possible de construire un deuxième point de concentration dès que $\sup _{M} \Phi_{\alpha} \rightarrow+\infty$ quand $\alpha \rightarrow+\infty$, deuxième point de concentration qui n'interagit pas au niveau énergétique avec le premier et qui apporte son quantum d'énergie. Le processus peut ainsi se continuer et est certain de s'arrêter faute d'énergie disponible. En suivant ce schéma de preuve, le résultat obtenu est le suivant : il existe $N \in \mathbb{N}^{\star}$ et $N$ suites de points $\left(x_{i, \alpha}\right)_{i=1, \ldots, N}$ convergentes tels qu'après extraction d'une sous-suite, les assertions suivantes soient vérifiées :

(i) $\frac{d_{g}\left(x_{i, \alpha}, x_{j, \alpha}\right)}{\mu_{i, \alpha}} \rightarrow+\infty$ quand $\alpha \rightarrow+\infty$ pour tous $i, j \in\{1, \ldots, N\}$, $i \neq j$, avec

$$
\mu_{i, \alpha}^{4}=f_{\alpha}\left(x_{i, \alpha}\right)^{-1} e^{-u_{\alpha}\left(x_{i, \alpha}\right)} \rightarrow 0 \text { quand } \alpha \rightarrow+\infty
$$

(ii) $u_{\alpha}\left(\exp _{x_{i, \alpha}}\left(\mu_{i, \alpha} x\right)\right)-u_{\alpha}\left(x_{i, \alpha}\right) \rightarrow V$ dans $C_{l o c}^{2}\left(\mathbb{R}^{4}\right)$ quand $\alpha \rightarrow+\infty$ pour tout $1 \leq i \leq N$ où $V$ est comme ci-dessus. En conséquence de quoi,

$$
\lim _{R \rightarrow+\infty} \lim _{\alpha \rightarrow+\infty} \int_{B_{x_{i, \alpha}}\left(R \mu_{i, \alpha}\right)} f_{\alpha} e^{u_{\alpha}} d v_{g}=64 \pi^{2}
$$

pour tout $1 \leq i \leq N$.

(iii) La quantité $\left(\min _{i=1, \ldots, N} d_{g}\left(x_{i, \alpha}, x\right)\right)^{4} e^{u_{\alpha}(x)}$ est uniformément bornée en $\alpha$ et $x$

25. Dans certains cas, il n'est pas possible de s'en sortir comme cela et il faut alors faire face à une multitude de profils asymptotiques, cf. [86]. 
Par effet régularisant des EDPs elliptiques, l'estimée ponctuelle (iii) passe aux gradients successifs de $u_{\alpha}$ et la quantité $\left(\min _{i=1, \ldots, N} d_{g}\left(x_{i, \alpha}, x\right)\right)^{k}$ - $\left|\nabla^{k} u_{\alpha}\right|_{g}$ est uniformément bornée en $\alpha$ et $x$ pour $k=1,2,3$.

Evidemment, cette estimée ponctuelle faible est juste insuffisante pour conclure que les points de concentration prennent toute l'énergie par théorème de convergence dominée de Lebesgue. Il faut donc réussir à améliorer un petit peu cette estimée, au moins dans un voisinage des points de concentration. Le problème ici est que l'opérateur $\Delta_{g}^{2}-d i v_{g}\left(A_{\alpha} d\right.$.) ne vérifie pas de principe du maximum. Par contre, l'estimée ci-dessus sur les gradients donne directement que les fonctions $\left(u_{\alpha}\right)$ sont comparables à leurs moyennes sur les sphères centrées en un point de concentration, tout du moins au voisinage de celui-ci. Obtenir des estimées sur les fonctions $\left(u_{\alpha}\right)$ revient donc à obtenir des estimées sur leurs moyennes. C'est en gros la stratégie utilisée dans la suite de la preuve. Nous ne la détaillerons pas dans ce texte.

Comparée à l'analyse ponctuelle du chapitre 3, celle-ci est infiniment plus simple. Le résultat de quantification est également plus simple à démontrer que celui de la section suivante. Il pourrait donc être intéressant de comprendre ce qui se passe si l'opérateur limite $\Delta_{g}^{2}-d i v_{g}\left(A_{0} d\right.$. ) a un noyau. En particulier, est-ce que le résultat de quantification reste vrai dans ce cadre? C'est à notre avis une des questions à regarder avant tout sur ce type d'équations où l'analyse semble plus simple.

\subsection{Une non-linéarité en $e^{u^{2}}$}

Dans cette section, nous allons illustrer sur un exemple encore plus frappant le type de résultats totalement inaccessibles par des méthodes d'énergie qu'il est possible d'obtenir grâce à l'approche ponctuelle développée dans le chapitre 3. Nous allons nous placer dans l'espace euclidien quoique un résultat analogue puisse sans aucun doute être démontré sur des espaces non-homogènes puisque la preuve n'utilise les symétries de l'espace euclidien que pour des raisons techniques ${ }^{26}$.

Considérons $\Omega$ un ouvert borné de $\mathbb{R}^{2}$ et une équation du type

$$
\Delta_{\xi} u=f(x, u) \text { dans } \Omega, u>0 \text { dans } \Omega, u=0 \text { sur } \partial \Omega,
$$

où $f$ est une non-linéarité exponentielle critique. Pour fixer les idées, prenons

$$
f(x, t)=h(x) t e^{4 \pi t^{2}} .
$$

26. Cette analyse a été étendue au cadre riemannien, dans un cadre d'énergie minimale, par Yang dans [102]. 
La constante $4 \pi$ n'est pas très importante et n'est là que pour des questions ultérieures de normalisation agréable; le point important est que la nonlinéarité croisse en $e^{u^{2}}$, ce qui est en quelque sorte la vraie ${ }^{27}$ non-linéarité critique en dimension 2 au vu de l'inégalité de Trudinger-Moser. Pour l'exemple de non-linéarité $f$ pris, les solutions de l'équation sont des points critiques (strictement positifs) de la fonctionnelle

$$
J(u)=\frac{1}{2} \int_{\Omega}|\nabla u|^{2} d x-\frac{1}{8 \pi} \int_{\Omega} h(x) e^{4 \pi u^{2}} d x
$$

définie et régulière (grâce à l'inégalité de Trudinger-Moser) sur $H_{0}^{1}(\Omega)$. Mais cette fonctionnelle ne satisfait pas la condition de Palais-Smale (globalement) - ceci n'est pas surprenant puisque l'inégalité de TrudingerMoser a un aspect critique - et des suites de solutions de l'équation (4.13) peuvent développer des phénomènes de concentration.

Cette équation présente de fortes analogies avec l'équation de type Yamabe étudiée au chapitre 3 . Il est donc naturel de se demander s'il est possible de décrire une suite de solutions de cette équation dans des espaces d'énergie, obtenir une quantification des niveaux d'explosion (comme cela avait été fait par Struwe [94] pour l'équation de type Yamabe) voire obtenir des estimées ponctuelles analogues à celles décrites dans le chapitre 3 . La théorie ponctuelle pour cette équation semble inaccessible ${ }^{28}$ pour l'instant. Par contre, en utilisant certaines idées développées pour la théorie ponctuelle des équations de type Yamabe, il est possible d'obtenir des résultats de quantification et une décomposition dans l'espace d'énergie pour cette équation :

Théorème 4.8. [Druet, [37]] Soit $\Omega$ un domaine régulier de $\mathbb{R}^{2}$. Soit $\left(f_{\alpha}\right)$ une suite de fonctions à croissance critique uniforme dans $\Omega$. Soit $\left(u_{\alpha}\right)$ une suite de solutions de

$$
\Delta u_{\alpha}=f_{\alpha}\left(x, u_{\alpha}\right) \text { dans } \Omega, u_{\alpha}>0 \text { dans } \Omega, u_{\alpha}=0 \text { sur } \partial \Omega .
$$

Supposons que $J_{\alpha}\left(u_{\alpha}\right) \rightarrow \beta$ quand $\alpha \rightarrow+\infty$ où $J$ est la fonctionnelle d'énergie associée à l'équation, i.e.

$$
J_{\alpha}(u)=\frac{1}{2} \int_{\Omega}|\nabla u|^{2} d x-\int_{\Omega}\left(\int_{0}^{u(x)} f_{\alpha}(x, t) d t\right) d x .
$$

27. Elle est en tout cas "plus critique" et plus difficile à comprendre que la nonlinéarité en $e^{u}$ qui présente également des défauts de compacité et que nous avons traité dans le paragraphe précédent avec une équation en bi-laplacien. L'analyse asymptotique pour les équations de courbure scalaire prescrite en dimension 2, i.e. avec une non-linéarité $e^{u}$, a été très largement étudiée ces 20 dernières années. Voici quelques références sur le sujet : [18, 24,67]. Le lecteur trouvera une liste plus étoffée dans l'article de survol de Lin [72].

28. ou, tout du moins, le chemin pour y parvenir est escarpé et semé d'embûches. 
Alors il existe $u_{\infty} \in H_{0}^{1}(\Omega)$ solution de l'équation-limite $\Delta u_{\infty}=f_{\infty}\left(x, u_{\infty}\right)$ avec condition de Dirichlet au bord, éventuellement nulle, et un entier $N$ tels que, après extraction d'une sous-suite,

$$
\beta=J_{\infty}\left(u_{\infty}\right)+\frac{N}{2} .
$$

Enoncé ainsi, ceci est un résultat de quantification des niveaux d'explo$\operatorname{sion}^{29}$ de la suite $\left(u_{\alpha}\right)$. En fait, la preuve fournit également une décomposition de $u_{\alpha}$ dans l'espace $H_{0}^{1}(\Omega)$ en termes de bulles standard mais la description en est un peu compliquée.

Qu'est-ce qu'une suite de fonctions à croissance critique uniforme dans $\Omega$ ? Pour une définition précise, nous renvoyons à [37]. Il suffit de savoir que cela signifie en gros que $f_{\alpha}(x, t)$ se comporte comme $e^{4 \pi t^{2}}$ pour $t$ grand uniformément en $x$ et $\alpha$ et que la suite converge vers une limite $f_{\infty}$. Cette classe comporte en particulier toutes les suites de fonctions du type

$$
f_{\alpha}(x, t)=h_{\alpha}(x) P_{\alpha}(t) e^{4 \pi t^{2}}
$$

à condition que $\left(h_{\alpha}\right)$ soit une suite de fonctions régulières strictement positives convergente dans $C^{1}$ et que $P_{\alpha}$ vérifie les propriétés suivantes : $P_{\alpha}$ est régulière, impaire et strictement positive dès que $t>0$ et est à croissance sous-critique. Une suite convergente de polynômes convient, ou même des termes en $e^{t^{\eta}}$ pour $\eta<2$. Pour la discussion qui suit, nous nous contenterons de prendre des suites de fonctions $\left(f_{\varepsilon}\right)$ indépendantes de la variable $x$ car cela simplifie beaucoup les notations.

Ce théorème répond à une question naturelle, au vu de ce qui se passe pour les équations de type Yamabe, ou pour les équations avec non-linéarité exponentielle en dimension 2, mais a en fait une histoire assez courte, faute d'outils disponibles jusqu'à récemment. Adimurthi et Struwe [3] l'ont démontré dans le cas où il est certain que $N=1$ et $u_{0} \equiv 0$. Ils font une hypothèse sur $\beta$ qui rend exclusive l'alternative $u_{0} \not \equiv 0$ ou développement d'un phénomène de concentration et qui interdit également le développement de plusieurs bulles. De plus, ils se restreignent à une classe très spéciale de non-linéarités $\left(f_{\varepsilon}\right)$ (qui, en particulier, ne dépendent pas de $x$ ). Le théorème ci-dessus répond d'ailleurs à une question explicitement posée dans [3]. Leur apport essentiel est d'avoir découvert le changement d'échelle, un peu étrange au premier abord, adapté à la compréhension du phénomène d'explosion. Par contre, la méthode utilisée étant essentiellement de type énergie, ils étaient contraints de s'arrêter à la première bulle. En effet, pour détecter une éventuelle deuxième bulle, cette méthode demande de soustraire la première bulle à la solution mais, ici, l'équation vérifiée par cette nouvelle suite change dramatiquement de nature (à cause

29. Comparer à (3.4). 
de la non-linéarité sauvage). Il semblerait de plus, mais c'est un travail à faire, que ce résultat de quantification soit faux pour des suites de PalaisSmale. C'est le travail de Adimurthi et Prashanth [2] qui exhibe deux types de profil asymptotique pour l'explosion de suites de Palais-Smale qui appuie cette intuition. Il devrait en effet être possible de combiner ces deux types de profil pour violer la quantification pour des suites de Palais-Smale.

Dans les paragraphes qui suivent, nous allons esquisser la preuve du théorème 4.8 en comparant celle-ci à la preuve du théorème 3.1 .

\subsubsection{Le premier point de concentration}

Dans toute la suite, et pour avoir une bonne idée de ce qui se passe, il faut penser à une non-linéarité

$$
f_{\alpha}(x, t)=t e^{4 \pi t^{2}}
$$

Quoique très restrictif, cet exemple est significatif.

Tout d'abord, un simple argument permet de montrer que l'hypothèse du théorème " $J_{\alpha}\left(u_{\alpha}\right) \rightarrow \beta$ quand $\alpha \rightarrow+\infty$ " donne une borne a priori sur la norme $L^{2}$ du gradient de $u_{\alpha}$. Ainsi, la suite $\left(u_{\alpha}\right)$ est uniformément bornée dans $H_{0}^{1}(\Omega)$ et, à extraction d'une sous-suite près, elle converge faiblement dans $H_{0}^{1}(\Omega)$ vers une solution $u_{\infty}$, éventuellement nulle, de l'équation-limite. Si jamais la suite $\left(u_{\alpha}\right)$ était uniformément bornée dans $L^{\infty}(\Omega)$, les plongements compacts de Sobolev donneraient immédiatement que la convergence est forte (et a même lieu dans $C^{0}$ ) et donc le résultat de quantification avec $N=0$. Nous supposerons donc dorénavant que

$$
\sup _{\Omega} u_{\alpha} \rightarrow+\infty \text { quand } \alpha \rightarrow+\infty \text {. }
$$

Prenons un point $x_{\alpha} \in \Omega$ tel que $u_{\alpha}\left(x_{\alpha}\right)=\sup _{\Omega} u_{\alpha}$. L'objectif est de trouver le changement d'échelle adéquat, i.e. de régler correctement le microscope, afin de voir ce qui se passe au voisinage de ce point. Soit une suite $\left(\mu_{\alpha}\right)$ de réels strictement positifs convergeant vers 0 . Posons

$$
v_{\alpha}(x)=u_{\alpha}\left(x_{\alpha}+\mu_{\alpha} x\right)
$$

définie sur $\Omega_{\alpha}=\left\{x \in \mathbb{R}^{n}\right.$ t.q. $\left.x_{\alpha}+\mu_{\alpha} x \in \Omega\right\}$. Il est clair qu'à extraction près, $\Omega_{\alpha}$ converge soit vers l'espace $\mathbb{R}^{2}$ tout entier - ce qui correspond à $d\left(x_{\alpha}, \partial \Omega\right) \gg \mu_{\alpha}$ - soit, après une rotation qui ne change rien au problème, vers un demi-espace $\left[R_{0},+\infty\right) \times \mathbb{R}$ - ce qui correspond à $\frac{d\left(x_{\alpha}, \partial \Omega\right)}{\mu_{\alpha}} \rightarrow R_{0}$ quand $\alpha \rightarrow+\infty$. L'équation vérifiée par $v_{\alpha}$ est

$$
\Delta v_{\alpha}=\mu_{\alpha}^{2} f_{\alpha}\left(v_{\alpha}\right)
$$


La suite $\left(v_{\alpha}\right)$ vérifie également $v_{\alpha} \leq v_{\alpha}(0)$ dans $\Omega_{\alpha}$. Il faut maintenant écraser notre suite $\left(v_{\alpha}\right)$. Pour des non-linéarités exponentielles, l'écrasement ne se fait pas par division par le maximum mais par retranchement de celui-ci. Ecrivons alors que

$$
\Delta\left(v_{\alpha}-v_{\alpha}(0)\right)=\mu_{\alpha}^{2} f_{\alpha}\left(v_{\alpha}\right) .
$$

La seule chance d'obtenir de la convergence est de prendre $\mu_{\alpha}$ suffisamment petit pour que le terme de droite soit uniformément borné. Comme la suite $\left(f_{\alpha}\right)$ est uniformément croissante pour $t$ grand et tend vers $+\infty$ lorsque $t \rightarrow+\infty, f_{\alpha}\left(v_{\alpha}\right) \leq f_{\alpha}\left(v_{\alpha}(0)\right)$ dans $\Omega_{\alpha}$ pour $\alpha$ grand. Une première idée pourrait être de prendre $\mu_{\alpha}^{2}=\frac{1}{f_{\alpha}\left(v_{\alpha}(0)\right)}$. Malheureusement, à cause de la présence du carré dans l'exponentielle, si jamais $\left(v_{\alpha}-v_{\alpha}(0)\right)$ convergeait avec ce choix de $\mu_{\alpha}, \mu_{\alpha}^{2} f_{\alpha}\left(v_{\alpha}\right)$ ne convergerait pas mais se comporterait comme $e^{8 \pi v_{\alpha}(0)\left(v_{\alpha}-v_{\alpha}(0)\right)}$. Il est clair qu'un passage à la limite serait ici inenvisageable. Il faudra donc choisir $\mu_{\alpha}^{2} \ll \frac{1}{f_{\alpha}\left(v_{\alpha}(0)\right)}$. Mais alors $v_{\alpha}-v_{\alpha}(0)$ a tendance à devenir harmonique. Il est d'ailleurs possible de montrer qu'elle converge, à extraction près, vers une fonction harmonique négative ou nulle sur l'espace tout entier ${ }^{30}$, valant 0 en 0 . D'après le théorème de Liouville, elle converge donc vers 0 . D'où l'idée suivante : remultiplier cette suite $\left(v_{\alpha}-v_{\alpha}(0)\right)$ par un certain $\lambda_{\alpha} \rightarrow+\infty$ afin de garder de la convergence mais vers une fonction non triviale. Pour résumer, les arguments, partiellement heuristiques, ci-dessus suggèrent de poser

$$
w_{\alpha}=\lambda_{\alpha}\left(u_{\alpha}\left(x_{\alpha}+\mu_{\alpha} x\right)-u_{\alpha}\left(x_{\alpha}\right)\right)
$$

sur $\Omega_{\alpha}$ avec $\mu_{\alpha}^{2} \ll \frac{1}{f_{\alpha}\left(v_{\alpha}(0)\right)}$ et $\lambda_{\alpha} \rightarrow+\infty$ quand $\alpha \rightarrow+\infty$. Imaginons un instant que nous soyons dans le meilleur des mondes et que cette suite converge dans $C_{l o c}^{0}\left(\mathbb{R}^{2}\right)$ vers une fonction $w$ non triviale. A quelles conditions un passage à la limite dans l'équation

$$
\Delta w_{\alpha}=\lambda_{\alpha} \mu_{\alpha}^{2} f_{\alpha}\left(u_{\alpha}\left(x_{\alpha}\right)+\lambda_{\alpha}^{-1} w_{\alpha}\right)
$$

est-il envisageable? La réponse se lit très bien sur l'exemple simple $f_{\alpha}(t)=$ $t e^{4 \pi t^{2}}$ puisque, dans ce cas,

$$
\begin{aligned}
& f_{\alpha}\left(u_{\alpha}\left(x_{\alpha}\right)+\lambda_{\alpha}^{-1} w_{\alpha}\right)=\left(u_{\alpha}\left(x_{\alpha}\right)+\lambda_{\alpha}^{-1} w_{\alpha}\right) e^{4 \pi\left(u_{\alpha}\left(x_{\alpha}\right)+\lambda_{\alpha} w_{\alpha}\right)^{2}} \\
& =\left(u_{\alpha}\left(x_{\alpha}\right)+o(1)\right) e^{4 \pi u_{\alpha}\left(x_{\alpha}\right)^{2}} e^{8 \pi u_{\alpha}\left(x_{\alpha}\right) \lambda_{\alpha}^{-1}(w+o(1))} e^{4 \pi \lambda_{\alpha}^{-2} w_{\alpha}^{2}} \\
& =(1+o(1)) u_{\alpha}\left(x_{\alpha}\right) e^{4 \pi u_{\alpha}\left(x_{\alpha}\right)^{2}} e^{8 \pi u_{\alpha}\left(x_{\alpha}\right) \lambda_{\alpha}^{-1}(w+o(1))} .
\end{aligned}
$$

Pour que cette quantité converge lorsque multipliée par $\lambda_{\alpha} \mu_{\alpha}^{2}$, le choix de $\lambda_{\alpha}$ et $\mu_{\alpha}$ s'imposent : il faut prendre $\lambda_{\alpha}=8 \pi u_{\alpha}\left(x_{\alpha}\right)$ et $\mu_{\alpha}^{2}=$

30. C'est à ce moment-là qu'est éliminée la convergence de $\Omega_{\alpha}$ vers un demi-espace, l'idée essentielle étant que $v_{\alpha}-v_{\alpha}(0)$ devrait y devenir une fonction harmonique, nulle en 0 , et tendant vers $-\infty$ sur le bord de ce demi-espace, ce qui est impossible. 
$u_{\alpha}\left(x_{\alpha}\right)^{2} e^{4 \pi u_{\alpha}\left(x_{\alpha}\right)^{2}}=u_{\alpha}\left(x_{\alpha}\right) f_{\alpha}\left(u_{\alpha}\left(x_{\alpha}\right)\right)$. C'est en effet un bon choix et le résultat suivant a lieu : à extraction d'une sous-suite près, $w_{\alpha}=$ $8 \pi u_{\alpha}\left(x_{\alpha}\right)\left(u_{\alpha}\left(x_{\alpha}+\mu_{\alpha} x\right)-u_{\alpha}\left(x_{\alpha}\right)\right) \rightarrow W$ dans $C_{\text {loc }}^{2}\left(\mathbb{R}^{2}\right)$ quand $\alpha \rightarrow$ $+\infty$ où $W \leq 0, W \not \equiv 0$, vérifie

$$
\Delta W=8 \pi e^{W} \text { dans } \mathbb{R}^{2}
$$

et $W(0)=0$. Il reste à montrer que $e^{W} \in L^{1}\left(\mathbb{R}^{2}\right)$, ce qui vient de la borne sur l'énergie de $\left(u_{\alpha}\right)$, pour conclure, grâce au théorème de classification de Chen et Li [25], que

$$
W(x)=-2 \ln \left(1+\pi|x|^{2}\right) .
$$

Ceci donne un premier point de concentration. En particulier, nous avons l'estimée d'énergie suivante :

$$
\lim _{R \rightarrow+\infty} \lim _{\alpha \rightarrow+\infty} \int_{B_{x_{\alpha}}\left(R \mu_{\alpha}\right)} u_{\alpha} f_{\alpha}\left(u_{\alpha}\right) d x=\int_{\mathbb{R}^{2}} e^{W} d x=1 .
$$

Il faut remarquer ici que, contrairement au cas des non-linéarités critiques en puissance en dimensions supérieures, la norme $L^{2}$ du gradient ne se concentre pas tout-à-fait au même endroit - et à une vitesse différente que l'intégrale de $u_{\alpha} f_{\alpha}\left(u_{\alpha}\right)$. Globalement, ces deux quantités sont égales mais, par exemple,

$$
\lim _{\alpha \rightarrow+\infty} \int_{B_{x_{\alpha}}\left(R \mu_{\alpha}\right)}\left|\nabla u_{\alpha}\right|^{2} d x \rightarrow 0
$$

quand $\alpha \rightarrow+\infty$ pour tout $R>0$. En fait, la norme $L^{2}$ du gradient se concentre sur un anneau autour de $x_{\alpha}$ et de manière diffuse ${ }^{31}$. Une difficulté non négligeable de la preuve du résultat de quantification est la détection des vitesses de concentration de cette norme.

\subsubsection{Comment trouver d'autres points de concentra- tion?}

Afin de trouver d'autres points de concentration, et selon l'idée développée dans le chapitre 3 , il faut trouver une estimée ponctuelle qui possède les mêmes invariances d'échelle que l'équation. Parler ici d'invariance d'échelle de l'équation est un léger abus si on pense au changement drastique de la nature de l'équation après le changement d'échelle opéré ci-dessus. Mais l'idée reste la même. Cette estimée ponctuelle est donnée par la quantité

$$
\Phi_{\alpha}(x)=\left|x_{\alpha}-x\right|^{2} u_{\alpha}(x) f_{\alpha}\left(u_{\alpha}(x)\right) .
$$

31. aucun changement d'échelle par dilatation ne donne quoique ce soit de visible pour cette norme. 
Pourquoi cette quantité est-elle invariante par le changement d'échelle de la sous-section précédente? C'est un tout petit peu délicat à voir. Le mieux est de réécrire $\Phi_{\alpha}$ sous la forme

$$
\Phi_{\alpha}(x)=\left|x_{\alpha}-x\right|^{2} u_{\alpha}(x) \Delta u_{\alpha}(x) .
$$

Ainsi,

$$
\begin{aligned}
\Phi_{\alpha}\left(x_{\alpha}+\mu_{\alpha} x\right) & =\mu_{\alpha}^{2}|x|^{2} u_{\alpha}\left(x_{\alpha}+\mu_{\alpha} x\right) \Delta u_{\alpha}\left(x_{\alpha}+\mu_{\alpha} x\right) \\
& =|x|^{2} \frac{u_{\alpha}\left(x_{\alpha}+\mu_{\alpha} x\right)}{8 \pi u_{\alpha}\left(x_{\alpha}\right)} \Delta w_{\alpha}(x)
\end{aligned}
$$

où $w_{\alpha}$ est la fonction renormalisée de la sous-section précédente. Il suffit ensuite de se convaincre que $\frac{u_{\alpha}\left(x_{\alpha}+\mu_{\alpha} x\right)}{u_{\alpha}\left(x_{\alpha}\right)}$ reste proche de 1 et que $w_{\alpha}$ est bornée sur une certaine distance pour admettre que cette estimée est "invariante" par le changement d'échelle de la sous-section précédente. Toujours est-il que cette estimée joue le rôle attendu d'elle puisqu'elle permet de détecter de nouveaux points de concentration.

Nous ne reprendrons pas les arguments en détail mais, si $\sup \Phi_{\alpha} \rightarrow+\infty$ quand $\alpha \rightarrow+\infty$, alors un point de maximum de $\Phi_{\alpha}$ donne un deuxième point de concentration qui apporte sa contribution quantifiée d'énergie et n'interagit pas avec le précédent (au niveau énergétique). Le processus peut alors être continué jusqu'à épuisement, ce qui ne manquera de se produire puisque l'énergie totale de la suite $\left(u_{\alpha}\right)$ est uniformément bornée. Cette étape correspond à l'étape de "détection des bulles hautes" dans la théorie ponctuelle pour les équations de Yamabe. Malheureusement, c'est un des points faibles de cette preuve de quantification - et aussi ce qui la rend très longue - il semble délicat de détecter les "bulles basses" par une méthode ponctuelle. Nous sommes ainsi contraints de saturer en quelque sorte le nombre de bulles que nous obtenons artificiellement. Plus clairement, par la méthode ponctuelle ci-dessus, un certain nombre $k$ de suites $\left(x_{i, \alpha}, \mu_{i, \alpha}\right)_{i=1, \ldots, k}$ avec les propriétés (a), (b) et (c) énoncées ci-dessous ont été trouvées (à extraction d'une sous-suite près). L'idée est ensuite de saturer le procédé en ajoutant, si elles existent, de nouvelles suites telles que leur ajout ne viole pas les propriétés (a), (b) et (c) ci-dessous. Et ce jusqu'à ce que ce soit impossible, ce qui arrivera nécessairement puisque la suite $\left(u_{\alpha}\right)$ a une énergie uniformément bornée. Nous arrivons alors au résultat suivant :

Proposition 4.2. Soit $\Omega$ un domaine régulier de $\mathbb{R}^{2}$. Soit $\left(f_{\alpha}\right)$ une suite de fonctions à croissance critique uniforme dans $\Omega$. Soit $\left(u_{\alpha}\right)$ une suite de solutions de

$$
\Delta u_{\alpha}=f_{\alpha}\left(x, u_{\alpha}\right) \text { dans } \Omega, u_{\alpha}>0 \text { dans } \Omega, u_{\alpha}=0 \text { sur } \partial \Omega .
$$


On suppose que la suite $\left(u_{\alpha}\right)$ est uniformément bornée dans $H_{0}^{1}(\Omega)$ et que $\sup _{\Omega} u_{\alpha} \rightarrow+\infty$ quand $\alpha \rightarrow+\infty$. Alors il existe $N \in \mathbb{N}^{\star}$, et il existe $N$ suites de points $\left(x_{i, \alpha}\right)_{i=1, \ldots, N}$ telles que, après extraction d'une sous-suite, les assertions suivantes aient lieu :

(a) Pour tous $i, j \in\{1, \ldots, N\}, i \neq j$,

$$
\lim _{\alpha \rightarrow+\infty} \frac{\left|x_{i, \alpha}-x_{j, \alpha}\right|}{\mu_{i, \alpha}}=+\infty
$$

ò̀ $\mu_{i, \alpha}^{-2}=u_{\alpha}\left(x_{i, \alpha}\right) f_{\alpha}\left(u_{\alpha}\left(x_{i, \alpha}\right)\right)$ et $\mu_{i, \alpha} \rightarrow 0$ quand $\alpha \rightarrow+\infty$.

(b) Pour tout $1 \leq i \leq N$,

$$
8 \pi u_{\alpha}\left(x_{i, \alpha}\right)\left(u_{\alpha}\left(x_{i, \alpha}+\mu_{i, \alpha} x\right)-u_{\alpha}\left(x_{i, \alpha}\right)\right) \rightarrow-2 \ln \left(1+\pi|x|^{2}\right)
$$

dans $C_{\text {loc }}^{1}\left(\mathbb{R}^{2} \backslash \mathcal{S}_{i}\right)$ quand $\alpha \rightarrow+\infty$ où

$$
\mathcal{S}_{i}=\left\{\lim _{\alpha \rightarrow+\infty} \frac{x_{j, \alpha}-x_{i, \alpha}}{\mu_{i, \alpha}}, j \neq i\right\} .
$$

(c) Il existe $C>0$ telle que

$$
\left(\min _{i=1, \ldots, N}\left|x_{i, \alpha}-x\right|\right)^{2} u_{\alpha}(x) f_{\alpha}\left(u_{\alpha}(x)\right) \leq C
$$

pour tout $x \in \Omega$ et tout $\alpha$.

De plus, étant donnée une suite de points $\left(x_{N+1, \alpha}\right)$, il est impossible d'extraire une sous-suite de la précédente de façon à ce que les trois propriétés ci-dessus aient lieu avec les suites $\left(x_{i, \alpha}\right)_{i=1, \ldots, N+1}$. Enfin,

$$
u_{\alpha} \rightarrow u_{0} \text { dans } C_{\text {loc }}^{1}(\bar{\Omega} \backslash \mathcal{S}) \text { quand } \alpha \rightarrow+\infty
$$

où $u_{0}$ est une solution de l'équation-limite, éventuellement nulle, et

$$
\mathcal{S}=\left\{\lim _{\alpha \rightarrow+\infty} x_{i, \alpha}, i=1, \ldots, N\right\} .
$$

Revenons un instant sur les raisons de l'échec de la méthode ponctuelle pour détecter ces éventuelles bulles basses. Celles-ci correspondent dans la proposition ci-dessus au cas où $\mathcal{S}_{i} \neq \emptyset$, c'est-à-dire au cas où les autres bulles déjà trouvées ne sont pas envoyées à l'infini par le changement d'échelle (cf. chapitre 3 pour l'analogue dans le cas des équations de Yamabe). En général, après changement d'échelle sur un tel point de concentration (qui serait détectable par une méthode ponctuelle comme dans le cas de l'équation de Yamabe), la suite converge vers une solution de l'équation

$$
\Delta_{\xi} U=8 \pi e^{U}
$$


dans $\mathbb{R}^{4}$ privé d'un certain nombre de points - les traces des bulles précédentes. De plus, $e^{U} \in L^{1}\left(\mathbb{R}^{4}\right)$. Mais cela ne suffit pas pour un résultat d'effacement des singularités éventuelles. En effet, comme démontré par Prajapat et Tarantello [85], il existe des solutions du problème ci-dessus qui sont singulières. Elles sont données, par exemple en supposant que la singularité est en 0 , par

$$
U_{\eta}=\ln \frac{(2-\eta)^{2}}{4 \pi|x|^{\eta}\left(1+|x|^{2-\eta}\right)^{2}}
$$

et sont solutions au sens faible de l'équation

$$
\Delta_{\xi} U_{\eta}=2 \pi \eta \delta_{0}+8 \pi e^{U_{\eta}}
$$

Dès que $0 \leq \eta<2, e^{U_{\eta}} \in L^{1}\left(\mathbb{R}^{4}\right)$ et cette fonction pourrait a priori apparaître comme limite de notre suite renormalisée. Rien ne peut éviter a priori l'apparition de ces solutions où il faut imaginer que l'interaction entre les bulles est telle qu'une bulle va jusqu'à influer sur la forme même d'une autre bulle, même dans le rayon d'action du microscope adapté. De plus, l'énergie portée par ces bulles pourrait être aussi petite qu'imaginable puisque

$$
\int_{\mathbb{R}^{2}} e^{U_{\eta}} d x=\frac{2-\eta}{2} .
$$

Ainsi, même en supposant le processus d'extraction de bulles lancé, rien ne pourrait assurer qu'il s'arrête.

En fait, ces bulles ne peuvent apparaître, en tout cas lorsque la suite $\left(u_{\alpha}\right)$ est réellement solution de l'équation et pas seulement suite de PalaisSmale associée à la fonctionnelle correspondante. Mais il faut travailler un peu plus pour le démontrer. C'est pour cela qu'il faut saturer la proposition en ajoutant artificiellement, sans procédé pour les détecter, ces bulles basses en supposant qu'elles ont la bonne forme. La tâche suivante est de démontrer qu'en effet, toutes les bulles ont été trouvées. C'est par ailleurs une excellente question, ouverte, que de savoir si ces bulles basses artificiellement ajoutées peuvent réellement apparaître. Il semblerait que cela soit assez compliqué à produire. Sur cette équation, le phénomène de bulles sur bulles semble peu probable, comme l'est le phénomène de bulles sur solution faible non-nulle. Mais ceci reste à prouver (ou à contredire).

\subsubsection{Toute l'énergie a-t-elle été trouvée?}

Maintenant que l'ensemble des points de concentration probable a été trouvé (cf. proposition 4.2), il s'agit de montrer qu'en effet, ce sont les seuls, et qu'il n'y a pas de perte d'énergie entre ces points de concentration pour arriver au résultat de quantification. Nous serons brefs sur le sujet car c'est de loin la partie la plus technique et ardue de la preuve. La première 
remarque à faire est que l'estimée faible obtenue dans la proposition 4.2 se transforme en une estimée sur le gradient de $u_{\alpha}$ : il existe une constante $C>0$ telle que

$$
\left(\min _{i=1, \ldots, N}\left|x_{i, \alpha}-x\right|\right) u_{\alpha}(x)\left|\nabla u_{\alpha}(x)\right| \leq C .
$$

La preuve de cette estimée faible sur le gradient n'est pas triviale; elle requiert un raisonnement par contradiction, une renormalisation adéquate et quelques arguments sur les fonctions harmoniques. Toujours est-il que cette estimée nous permet de comparer, au moins dans un voisinage proche des points de concentration, la fonction $u_{\alpha}$ à sa moyenne sur les sphères de centre le point de concentration. Plus précisément, posons

$$
\varphi_{i, \alpha}=\frac{1}{2 \pi r} \int_{\partial B_{x_{i, \alpha}}(r)} u_{\alpha} d \sigma
$$

pour $0 \leq r \leq \min _{j \neq i}\left|x_{i, \alpha}-x_{j, \alpha}\right|$ et $r \leq d\left(x_{i, \alpha}, \partial \Omega\right)$. Tant que $r$ est dans cette région, grâce à l'estimée faible sur le gradient,

$$
\frac{1}{D} f_{\alpha}\left(\varphi_{\alpha}\right) \leq \Delta \varphi_{\alpha} \leq D f_{\alpha}\left(\varphi_{\alpha}\right)
$$

pour une certaine constante $D>1$ indépendante de $\alpha$. Une étude de cette inéquation différentielle ordinaire permet d'obtenir quelques estimées intéressantes sur $u_{\alpha}$ et d'en déduire en particulier qu'aucune énergie n'est perdue dans cette zone. Une fois que ceci est fait sur tous les points de concentration, il faut les regrouper par deux ou plus (tous à la même distance les uns des autres) puis recommencer en considérant que ce groupe ne fait qu'un. Ainsi, la combinaison d'une hiérarchisation bien choisie des points de concentration et d'une étude d'une inéquation différentielle ordinaire permet de conclure que tous les points de concentration ont été trouvés. En particulier, et c'est le plus difficile, il est démontré qu'aucun point de concentration dégénéré ne peut apparaître. Ensuite, il faut démontrer qu'aucune énergie n'est perdue entre les points de concentration. La mise en place rigoureuse de ce vague schéma de preuve est assez technique et cette seule partie de la preuve court sur quelque 30 pages.

\subsubsection{Et après?}

Cette astuce de passer par les moyennes pour se ramener à l'étude d'équations (ou d'inéquations) différentielles ordinaires ${ }^{32}$ est certes jolie mais elle ne donne pas les estimées optimales que nous sommes en droit

\footnotetext{
32. déjà utilisée dans la section 4.4 et propre aux non-linéarités exponentielles (en dimension critique).
} 
d'attendre. Ces estimées sont nécessaires pour qui veut répondre aux questions assez naturelles qui se posent ici : les points de concentration sont-ils isolés comme c'est le cas pour les non-linéarités vraiment exponentielles (cf. section 4.4) ? Est-il possible d'avoir un phénomène d'explosion avec une limite faible non-nulle? Y a-t-il des non-linéarités $f_{0}$ pour lesquelles il n'y a pas d'explosion? Pour toutes ces questions et leur motivation, nous renvoyons au chapitre 2 .

Enfin, une question brûlante et intéressante est la suivante : la quantification est-elle vraie pour des suites de Palais-Smale? A notre avis non ${ }^{33}$ mais il faudrait une preuve. Ce serait une magnifique illustration, claire et nette, de la force de la méthode ponctuelle puisque, si la quantification n'est pas vraie pour des suites de Palais-Smale, la méthode d'énergie est nécessairement vouée à l'échec. Pour l'instant, nous ne pouvons que constater que celle-ci est inopérante mais une preuve de son inefficacité serait intéressante.

\subsubsection{Avec le bilaplacien ...}

Un résultat analogue de quantification a été obtenu par Struwe [95] pour le bilaplacien avec condition de Navier au bord. Soit $\Omega \subset \mathbb{R}^{4}$ un ouvert borné régulier. Considérons une suite de fonctions $\left(u_{\alpha}\right)$ solutions de

$$
\Delta^{2} u_{\alpha}=\lambda_{\alpha} u_{\alpha} e^{2 u_{\alpha}^{2}} \text { dans } \Omega, u_{\alpha}=\Delta u_{\alpha}=0 \text { sur } \partial \Omega .
$$

Théorème 4.9. [Struwe, [95]] Soit $\left(u_{\alpha}\right)$ une suite de solutions de (4.14) avec $\lambda_{\alpha} \rightarrow 0$ et

$$
\int_{\Omega}\left(\Delta u_{\alpha}\right)^{2} d x \rightarrow \Lambda
$$

quand $\alpha \rightarrow+\infty$, alors $\Lambda \in 16 \pi^{2} \mathbb{N}^{\star}$.

Remerciements : Je tiens à remercier Nassif Ghoussoub, Emmanuel Hebey et Michael Struwe pour les discussions intéressantes au cours de l'élaboration de ce texte.

Ce travail a bénéficié d'une aide de l'Agence Nationale de la Recherche portant la référence ANR-08-BLAN-0335-01.

33. cf. commentaire page 85 . 


\section{Bibliographie}

[1] M. Ablowitz, B. Prinari et A. Trubatch - Discrete and continuous nonlinear Schrödinger systems, Cambridge University Press, Cambridge, 2004.

[2] Adimurthi et S. Prashanth - « Failure of Palais-Smale condition and blow-up analysis for the critical exponent problem in $\mathbf{R}^{2} \gg$, Proc. Indian Acad. Sci. Math. Sci. 107 (1997), no. 3, p. 283-317.

[3] Adimurthi et M. Struwe $-\ll$ Global compactness properties of semilinear elliptic equations with critical exponential growth $\gg, J$. Funct. Anal. 175 (2000), no. 1, p. 125-167.

[4] N. Akhmediev et A. AnkIEwiCZ - «Partially coherent solitons on a finite background $\gg$, Phys. Rev. Lett. 82 (1998), p. 2661-2664.

[5] A. D. Aleksandrov $-\ll$ Uniqueness theorems for surfaces in the large. I $\gg$, Vestnik Leningrad. Univ. 11 (1956), no. 19, p. 5-17.

[6] T. Aubin - «Équations différentielles non linéaires et problème de Yamabe concernant la courbure scalaire $\gg$, J. Math. Pures Appl. (9) 55 (1976), no. 3, p. 269-296.

[7] — , « Problèmes isopérimétriques et espaces de Sobolev », J. Differential Geometry 11 (1976), no. 4, p. 573-598.

[8] A. BAhri et J.-M. Coron $-\ll$ On a nonlinear elliptic equation involving the critical Sobolev exponent : the effect of the topology of the domain $\gg$, Comm. Pure Appl. Math. 41 (1988), no. 3, p. 253-294.

[9] R. BARTnik et J. IsEnBERG $-\ll$ The constraint equations $\gg$, The Einstein equations and the large scale behavior of gravitational fields, Birkhäuser, Basel, 2004, p. 1-38.

[10] R. Beig, P. Chruściel et R. Schoen $-\ll$ KIDs are non-generic $\gg$, Ann. Henri Poincaré 6 (2005), no. 1, p. 155-194.

[11] H. Berestycki, L. A. Caffarelli et L. NirenberG - « Symmetry for elliptic equations in a half space $\gg$, Boundary value problems for partial differential equations and applications, RMA Res. Notes Appl. Math., vol. 29, Masson, Paris, 1993, p. 27-42. 
[12] M. Berti et A. Malchiodi - « Non-compactness and multiplicity results for the Yamabe problem on $S^{n} \gg$, J. Funct. Anal. 180 (2001), no. 1, p. 210-241.

[13] G. Bianchi et H. Egnell $-\ll$ A note on the Sobolev inequality », J. Funct. Anal. 100 (1991), no. 1, p. 18-24.

[14] S. BRENDLE - «Blow-up phenomena for the Yamabe equation », J. Amer. Math. Soc. 21 (2008), no. 4, p. 951-979.

[15] — , « On the conformal scalar curvature equation and related problems $\gg$, Surveys in Differential Geometry (à paraître).

[16] S. Brendle et F. Marques - « Blow-up phenomena for the Yamabe equation II $\gg$, J. Differential Geometry (à paraître).

[17] H. BRÉZIS - « Elliptic equations with limiting Sobolev exponents the impact of topology », Comm. Pure Appl. Math. 39 (1986), no. S, suppl., p. S17-S39, Frontiers of the mathematical sciences : 1985 (New York, 1985).

[18] H. Brezis et F. MERLE - « Uniform estimates and blow-up behavior for solutions of $-\Delta u=V(x) e^{u}$ in two dimensions $\gg, C o m m$. Partial Differential Equations 16 (1991), no. 8-9, p. 1223-1253.

[19] H. Brézis et L. Nirenberg - « Positive solutions of nonlinear elliptic equations involving critical Sobolev exponents $\gg$, Comm. Pure Appl. Math. 36 (1983), no. 4, p. 437-477.

[20] H. Brezis et M. Willem - « On some nonlinear equations with critical exponents $\gg$, J. Funct. Anal. 255 (2008), no. 9, p. 2286-2298.

[21] J. Burke, J. Bohn, B. Esry et C. Greene $-\ll$ Hartree-Fock theory for double condensates », Phys. Rev. Lett. 78 (1997), p. 35943597.

[22] L. A. Caffarelli, B. Gidas et J. SprucK - « Asymptotic symmetry and local behavior of semilinear elliptic equations with critical Sobolev growth », Comm. Pure Appl. Math. 42 (1989), no. 3, p. 271297.

[23] S.-Y. A. CHANG $-\ll$ Conformal invariants and partial differential equations $\gg$, Bull. Amer. Math. Soc. (N.S.) 42 (2005), no. 3, p. 365393 (electronic)

[24] C.-C. Chen et C.-S. Lin - « Topological degree for a mean field equation on Riemann surfaces $\gg$, Comm. Pure Appl. Math. 56 (2003), no. 12 , p. $1667-1727$.

[25] W. X. Chen et C. LI - « Classification of solutions of some nonlinear elliptic equations $\gg$, Duke Math. J. 63 (1991), no. 3, p. 615-622.

[26] Y. Choquet-Bruhat, J. Isenberg et D. Pollack - « Applications of theorems of Jean Leray to the Einstein-scalar field equations », J. Fixed Point Theory Appl. 1 (2007), no. 1, p. 31-46. 
$[27]-$, « The constraint equations for the Einstein-scalar field system on compact manifolds », Classical Quantum Gravity 24 (2007), no. 4, p. 809-828.

[28] D. Christodoulides, T. Coskun, M. Mitchell et M. Segev - « Theory of incoherent self-focusing in biased photorefractive media , Phys. Rev. Lett. 78 (1997), p. 646-649.

[29] J.-M. Coron - « Topologie et cas limite des injections de Sobolev », C. R. Acad. Sci. Paris Sér. I Math. 299 (1984), no. 7, p. 209-212.

[30] G. Devillanova et S. Solimini $-\ll$ Concentration estimates and multiple solutions to elliptic problems at critical growth $\gg, A d v$. Differential Equations 7 (2002), no. 10, p. 1257-1280.

[31] — , « A multiplicity result for elliptic equations at critical growth in low dimension $\gg$, Commun. Contemp. Math. 5 (2003), no. 2, p. 171177.

[32] W. Ding, J. Jost, J. Li et G. WAng $-\ll$ Existence results for mean field equations $\gg$, Ann. Inst. H. Poincaré Anal. Non Linéaire 16 (1999), no. 5, p. 653-666.

[33] Z. DJADli et A. MALChiodi $-\ll$ Existence of conformal metrics with constant $Q$-curvature $\gg$, Ann. of Math. (2) 168 (2008), no. 3, p. $813-858$.

[34] O. DRUET - «Elliptic equations with critical Sobolev exponents in dimension $3 \gg$, Ann. Inst. H. Poincaré Anal. Non Linéaire 19 (2002), no. 2, p. 125-142.

[35] — , « From one bubble to several bubbles : the low-dimensional case $\gg$, J. Differential Geom. 63 (2003), no. 3, p. 399-473.

[36] — , « Compactness for Yamabe metrics in low dimensions $\gg$, Int. Math. Res. Not. (2004), no. 23, p. 1143-1191.

[37] — , « Multibumps analysis in dimension 2 : quantification of blow-up levels », Duke Math. J. 132 (2006), no. 2, p. 217-269.

[38] O. Druet et E. HeBEy - «Blow-up examples for second order elliptic PDEs of critical Sobolev growth $\gg$, Trans. Amer. Math. Soc. 357 (2005), no. 5, p. 1915-1929 (electronic).

[39] — , «Sharp asymptotics and compactness for local low energy solutions of critical elliptic systems in potential form $\gg$, Calc. Var. Partial Differential Equations 31 (2008), no. 2, p. 205-230.

[40] — , « Stability and instability for Einstein-scalar field Lichnerowicz equations on compact Riemannian manifolds $\gg$, Math. Z. 263 (2009), no. 1 , p. $33-67$.

[41] — , « Stability for strongly coupled critical elliptic systems in a fully inhomogeneous medium $\gg$, Analysis and $P D E$ (à paraître). 
[42] O. Druet, E. Hebey et F. Robert - Blow-up theory for elliptic PDEs in Riemannian geometry, Mathematical Notes, vol. 45, Princeton University Press, Princeton, NJ, 2004.

[43] O. Druet, E. HeBey et J. VÉToIS - « Bounded stability for strongly coupled critical elliptic systems below the geometric threshold of the conformal Laplacian $\gg$, J. Funct. Anal. 258 (2010), no. 3, p. 999 1059 .

[44] O. Druet et P. Laurain - « Stability of the Pohožaev obstruction in dimension $3 \gg$, J. Eur. Math. Soc. (JEMS) 12 (2010), no. 5, p. $1117-1149$.

[45] O. Druet et F. Robert - «Bubbling phenomena for fourth-order four-dimensional PDEs with exponential growth $\gg$, Proc. Amer. Math. Soc. 134 (2006), no. 3, p. 897-908 (electronic).

[46] Y. Fourès-BRuhat - «Théorème d'existence pour certains systèmes d'équations aux dérivées partielles non linéaires », Acta Math. 88 (1952), p. 141-225.

[47] B. Gidas, W. Ni et L. NirenberG $-\ll$ Symmetry and related properties via the maximum principle $\gg$, Comm. Math. Phys. 68 (1979), no. 3, p. 209-243.

[48] D. Gilbarg et N. S. Trudinger - Elliptic partial differential equations of second order, Classics in Mathematics, Springer-Verlag, Berlin, 2001, Reprint of the 1998 edition.

[49] R. Hamilton - « Three-manifolds with positive Ricci curvature », J. Differential Geom. 17 (1982), no. 2, p. 255-306.

[50] Q. HAN et F. LIN - Elliptic partial differential equations, Courant Lecture Notes in Mathematics, vol. 1, New York University Courant Institute of Mathematical Sciences, New York, 1997.

[51] Z.-C. HAN - « Asymptotic approach to singular solutions for nonlinear elliptic equations involving critical Sobolev exponent $\gg$, Ann. Inst. H. Poincaré Anal. Non Linéaire 8 (1991), no. 2, p. 159-174.

[52] E. HeBEY - « Nonlinear elliptic equations of critical Sobolev growth from a dynamical viewpoint $\gg$, Noncompact problems at the intersection of geometry, analysis, and topology, Contemp. Math., vol. 350, Amer. Math. Soc., Providence, RI, 2004, p. 115-125.

[53] — , « Critical elliptic systems in potential form $\gg, A d v$. Differential Equations 11 (2006), no. 5, p. 511-600.

[54] — , « Diagonal compactness for critical elliptic systems in potential form $\gg$, Comm. Partial Differential Equations 32 (2007), no. 10-12, p. $1837-1881$.

[55] E. Hebey, F. Pacard et D. Pollack $-\ll$ A variational analysis of Einstein-scalar field Lichnerowicz equations on compact Riemannian manifolds », Comm. Math. Phys. 278 (2008), no. 1, p. 117-132. 
[56] E. Hebey et M. VAugon - « The best constant problem in the Sobolev embedding theorem for complete Riemannian manifolds », Duke Math. J. 79 (1995), no. 1, p. 235-279.

[57] — , « Meilleures constantes dans le théorème d'inclusion de Sobolev », Ann. Inst. H. Poincaré Anal. Non Linéaire 13 (1996), no. 1, p. $57-93$.

[58] F. Hioe $-\ll$ Solitary waves for $N$ coupled nonlinear Schrödinger equations $\gg$, Phys. Rev. Lett. 82 (1999), p. 1152-1155.

[59] F. Hioe et T. SAlter - «Special set and solutions of coupled nonlinear Schrödinger equations $\gg$, J. Phys. A : Math. Gen. 35 (2002), p. 8913-8928.

[60] M. Holst, G. Nagy et G. Tsogtgerel - « Far-from-constant mean curvature solutions of Einstein's constraint equations with positive Yamabe metrics $\gg$, Phys. Rev. Lett. 100 (2008), no. 16, p. $161101,4$.

[61] T. KANNA et M. LAKSHMANAN - « Exact soliton solutions, shape changing collisions, and partially coherent solitons in coupled nonlinear Schrödinger equations $\gg$, Phys. Rev. Lett. 86 (2001), p. 50435046.

[62] J. KAZDAN et F. WARNER - « Remarks on some quasilinear elliptic equations $\gg$, Comm. Pure Appl. Math. 28 (1975), no. 5, p. 567-597.

[63] — , « Scalar curvature and conformal deformation of Riemannian structure $\gg$, J. Differential Geometry 10 (1975), p. 113-134.

[64] M. Khuri, F. Marques et R. SCHOEN $-\ll$ A compactness theorem for the Yamabe problem $\gg$, preprint, 2007.

[65] J. LeE et T. PArker - « The Yamabe problem $\gg$, Bull. Amer. Math. Soc. (N.S.) 17 (1987), no. 1, p. 37-91.

[66] J. LERAY - «Hyperbolic differential equations », mimeographed notes, Institute for Advanced Studies, 1953.

[67] Y. Li et I. SHAFRIR $-\ll$ Blow-up analysis for solutions of $-\Delta u=$ $V e^{u}$ in dimension two », Indiana Univ. Math. J. 43 (1994), no. 4, p. $1255-1270$.

[68] Y. Li et L. ZHANG - « Liouville-type theorems and Harnack-type inequalities for semilinear elliptic equations $\gg$, J. Anal. Math. 90 (2003), p. 27-87.

[69] — , « Compactness of solutions to the Yamabe problem. III », J. Funct. Anal. 245 (2007), no. 2, p. 438-474.

[70] Y. Li et M. ZHU - « Yamabe type equations on three-dimensional Riemannian manifolds $\gg$, Commun. Contemp. Math. 1 (1999), no. 1, p. $1-50$. 
[71] A. LiChNEROWICZ - «L'intégration des équations de la gravitation relativiste et le problème des $n$ corps $\gg$, J. Math. Pures Appl. (9) 23 (1944), p. 37-63.

[72] C.-S. LIN - «An expository survey on the recent development of mean field equations », Discrete Contin. Dyn. Syst. 19 (2007), no. 2, p. 387-410.

[73] C.-S. LIN et J. WEI $-\ll$ Sharp estimates for bubbling solutions of a fourth order mean field equation $\gg$, Ann. Sc. Norm. Super. Pisa Cl. Sci. (5) 6 (2007), no. 4, p. 599-630.

[74] C. LIN - «A classification of solutions of a conformally invariant fourth order equation in $\mathbf{R}^{n} \gg$, Comment. Math. Helv. 73 (1998), no. 2, p. 206-231.

[75] A. Malchiodi $-\ll$ Compactness of solutions to some geometric fourth-order equations $\gg$, J. Reine Angew. Math. 594 (2006), p. 137174.

[76] C. Margerin - «Géométrie conforme en dimension 4: ce que l'analyse nous apprend $\gg$, Astérisque (2006), no. 307, p. Exp. No. 950, ix-x, 415-468, Séminaire Bourbaki. Vol. 2004/2005.

[77] F. MARQUES - « A priori estimates for the Yamabe problem in the non-locally conformally flat case », J. Differential Geom. 71 (2005), no. 2 , p. $315-346$.

[78] D. MAXWELL - «A class of solutions of the vacuum Einstein constraint equations with freely specified mean curvature $\gg$, ArXiv 0804.0874, 2008.

[79] M. ОватA - « The conjectures on conformal transformations of Riemannian manifolds », J. Differential Geometry 6 (1971/72), p. 247258.

[80] G. Perelman - « The entropy formula for the Ricci flow and its geometric applications », ArXiv math.DG/0211159, 2002.

[81] — , «Finite extinction time for the solutions to the Ricci flow on certain three-manifolds $\gg$, ArXiv math.DG/0303109, 2003.

[82] — , « Ricci flow with surgery on three-manifolds », ArXiv math.DG/0303109, 2003.

[83] S. I. POHOŽAEV - «On the eigenfunctions of the equation $\Delta u+$ $\lambda f(u)=0 \gg$, Dokl. Akad. Nauk SSSR 165 (1965), p. 36-39.

[84] D. POLLACK - « Nonuniqueness and high energy solutions for a conformally invariant scalar equation $\gg$, Comm. Anal. Geom. 1 (1993), no. 3-4, p. 347-414.

[85] J. Prajapat et G. TARAntello $-\ll$ On a class of elliptic problems in $\mathbb{R}^{2}$ : symmetry and uniqueness results $\gg$, Proc. Roy. Soc. Edinburgh Sect. A 131 (2001), no. 4, p. 967-985. 
[86] F. RoBerT - « Concentration phenomena for a fourth-order equation with exponential growth : the radial case $\gg$, J. Differential Equations 231 (2006), no. 1, p. 135-164.

[87] R. Schoen - « Conformal deformation of a Riemannian metric to constant scalar curvature », J. Differential Geom. 20 (1984), no. 2, p. 479-495.

$[88]-$, « Lecture notes from courses at Stanford $\gg$, written by D. Pollack, 1988.

[89] — , « Variational theory for the total scalar curvature functional for Riemannian metrics and related topics $\gg$, Topics in calculus of variations (Montecatini Terme, 1987), Lecture Notes in Math., vol. 1365, Springer, Berlin, 1989, p. 120-154.

$[90]-, \ll$ On the number of constant scalar curvature metrics in a conformal class $\gg$, Differential geometry, Pitman Monogr. Surveys Pure Appl. Math., vol. 52, Longman Sci. Tech., Harlow, 1991, p. 311-320.

[91] R. Schoen et S.-T. YAU $-\ll$ Conformally flat manifolds, Kleinian groups and scalar curvature », Invent. Math. 92 (1988), no. 1, p. 4771.

[92] R. Schoen et S. YAU - « On the proof of the positive mass conjecture in general relativity $\gg$, Comm. Math. Phys. 65 (1979), no. 1, p. $45-76$.

[93] R. Schoen et D. ZhANG - « Prescribed scalar curvature on the $n$ sphere », Calc. Var. Partial Differential Equations 4 (1996), no. 1, p. $1-25$.

[94] M. Struwe - «A global compactness result for elliptic boundary value problems involving limiting nonlinearities $\gg$, Math. Z. 187 (1984), no. 4, p. 511-517.

[95] — , « Quantization for a fourth order equation with critical exponential growth $\gg$, Math. Z. 256 (2007), no. 2, p. 397-424.

[96] G. Talenti - «Best constant in Sobolev inequality », Ann. Mat. Pura Appl. (4) 110 (1976), p. 353-372.

[97] N. TRUdinger - « Remarks concerning the conformal deformation of Riemannian structures on compact manifolds $\gg$, Ann. Scuola Norm. Sup. Pisa (3) 22 (1968), p. 265-274.

[98] J. VÉTOIS - «Multiple solutions for nonlinear elliptic equations on compact Riemannian manifolds $\gg$, Internat. J. Math. 18 (2007), no. 9 , p. $1071-1111$.

[99] L. WANG, J. WEI et S. YAN - « On lin-ni's conjecture in convex domains $\gg$, Proc. London Math. Soc. (à paraître). 
[100] J. WEI et S. YAN - « Infinitely many solutions for the prescribed scalar curvature problem on $\mathbb{S}^{N} \gg$, J. Funct. Anal. 258 (2010), no. 9, p. 3048-3081.

[101] H. YAMABE - «On a deformation of Riemannian structures on compact manifolds », Osaka Math. J. 12 (1960), p. 21-37.

[102] Y. YANG - «A sharp form of the Moser-Trudinger inequality on a compact Riemannian surface $\gg$, Trans. Amer. Math. Soc. 359 (2007), no. 12 , p. 5761-5776 (electronic).

Olivier Druet

CNRS - ENS Lyon - Université de Lyon.

Unité de Mathématiques Pures et Appliquées (UMR 5669)

Ecole Normale Supérieure de Lyon

46, allée d'Italie

69364 Lyon Cedex 7 